Florida International University

FIU Digital Commons

FIU Electronic Theses and Dissertations

University Graduate School

$10-12-2017$

\title{
Quantification of Precipitation Asymmetries in Tropical Cyclones and Their Relationship to Storm Intensity Changes Using TRMM Data
}

Yongxian Pei

Florida International University, ypei002@fiu.edu

DOI: $10.25148 /$ etd.FIDC004018

Follow this and additional works at: https:// digitalcommons.fiu.edu/etd

Part of the Atmospheric Sciences Commons, and the Meteorology Commons

\section{Recommended Citation}

Pei, Yongxian, "Quantification of Precipitation Asymmetries in Tropical Cyclones and Their Relationship to Storm Intensity Changes Using TRMM Data" (2017). FIU Electronic Theses and Dissertations. 3548.

https://digitalcommons.fiu.edu/etd/3548

This work is brought to you for free and open access by the University Graduate School at FIU Digital Commons. It has been accepted for inclusion in FIU Electronic Theses and Dissertations by an authorized administrator of FIU Digital Commons. For more information, please contact dcc@fiu.edu. 


\section{FLORIDA INTERNATIONAL UNIVERSITY}

Miami, Florida

\section{QUANTIFICATION OF PRECIPITATION ASYMMETRIES IN TROPICAL CYCLONES AND THEIR RELATIONSHIP TO STORM INTENSITY CHANGES USING TRMM DATA}

A dissertation submitted in partial fulfillment of the requirements for the degree of DOCTOR OF PHILOSOPHY in

\section{GEOSCIENCES}

by

Yongxian Pei 
To: Dean Michael R. Heithaus

College of Arts, Sciences and Education

This dissertation, written by Yongxian Pei, and entitled Quantification of Precipitation Asymmetries in Tropical Cyclones and Their Relationship to Storm Intensity Changes Using TRMM Data, having been approved in respect to style and intellectual content, is referred to you for judgment.

We have read this dissertation and recommend that it be approved.

$\begin{array}{r}\text { Hugh Willoughby } \\ \hline \text { Ping Zhu } \\ \hline \text { Wei Wang } \\ \hline \text { Haiyan Jiang, Major Professor }\end{array}$

Date of Defense: October 12, 2017

The dissertation of Yongxian Pei is approved.

Dean Michael R. Heithaus

College of Arts, Sciences and Education

Andrés G. Gil

Vice President for Research and Economic Development and Dean of the University Graduate School

Florida International University, 2017 


\section{ACKNOWLEDGMENTS}

I would like to thank my $\mathrm{PhD}$ advisor Dr. Haiyan Jiang for supporting me during the past five years. I am very grateful to her scientific advice, knowledge, insightful discussions, and suggestions. Her dedication and guidance helped me in all the time of research and writing of this dissertation.

I would like to give my gratitude to the rest of my dissertation committee: Dr. Hugh Willoughby, Dr. Ping Zhu and Dr. Wei Wang, for their insightful comments and encouragement, but also for critical questions which provided me resourceful ideas to widen my research. Besides my committee members, I would like to thank Drs. Jonathan Zawislak, Cheng Tao, Brad Klotz, Cen Gao, Yubin Li, and Joseph Zagrodnik for sharing their knowledge of writing, programming, and researching.

Special appreciation is given to Dr. Shuyi Chen, Dr. Angela Rowe, and the committee of Robert A. Houze, Jr. Symopsium for financially providing the opportunity to attend the symposium and bringing up vital discussions about this work.

My appreciation also goes to my fellow graduate students including Peter Washam, Margie Kieper, Michael Fischer, Tyler Wieland, Heather Vazquez, Israel Gonzalez, Javiera Hernandez, Zongyao Yang, Jeremy Katz, and Xinxi Wang. They have brought up interesting discussions, provided helps on this project, and graduate coursework.

I would also like to thank my parents, Yan Yang and Zhenlin Pei for their continuous support and encouragement. I'd like to express my gratitude to my friends, Timothy Parry, Yixian He, Bo Dong, Yachong Guo, Isaac Wang, Kévin and Carolina Bretécher, Joshua and Julie Anderson, Phil Warthen, Christine Selby, Dylan Taylor, Tao Wang, Miao Pang, Qi Zhou, Li Tang, Tingjun Lei, Yu Bi, Mauricio Valdez Jr, Anthony 
Bart, Meng Gao, Jianhao Zhang, Alyson Murray, Stephanie Jaimes, Emma Fu, Yi Wo for their help, support, and friendships. Last but not least, I thank God for the knowns and unknowns.

This work is supported financially by the NASA Earth and Space Science Fellowship (NESSF) award and the NASA New Investigator Program (NIP) Award NNX10AG55G and NASA Hurricane Science Research Program (HSRP) Grant NNX10AG34G under the direction of Drs. Ramesh Kakar and Ming-Ying Wei, and NOAA Joint Hurricane Testbed (JHT) Grants NA11OAR4310193, NA13OAR4590191, and NA15OAR4590199 under the direction of Dr. Chris Landsea. Specifically, I thank Drs. Ramesh Kakar, Ming-Ying Wei (NASA headquarters), and Dr. Chris Landsea. for their continued support of TRMM and hurricane sciences. 


\begin{abstract}
OF THE DISSERTATION
QUANTIFICATION OF PRECIPITATION ASYMMETRIES IN TROPICAL

CYCLONES AND THEIR RELATIONSHIP TO STORM INTENSITY CHANGES
\end{abstract}

USING TRMM DATA

\author{
By \\ Yongxian Pei \\ Florida International University, 2017 \\ Miami, Florida \\ Professor Haiyan Jiang, Major Professor
}

The climatology of precipitation asymmetries in Tropical Cyclones (TCs) and their relationship to TC intensity changes using 16 years of data from the Tropical Rainfall Measuring Mission (TRMM) satellite. TC Inner core precipitation asymmetries were quantified using the Fourier wavenumber decomposition method upon the pixel level data of 3,542 TRMM TMI overpasses. Composites of wavenumber-1 and wavenumber 1-6 total precipitation asymmetries were constructed to show the distribution pattern under different storm motion speed, vertical wind shear and the combined effects of varying vertical wind shear and storm motion. Results indicate that motion-relative total precipitation asymmetry is located down-motion. The phase of motion-relative maximum asymmetry shifts cyclonically by adding the wavenumber-2-6 asymmetry to wavenumber- 1 . Shear is more dominant than motion on the distribution of precipitation asymmetry. The analysis of combined effects of motion and shear shows when shear is weak, and shear is to the left of motion, the precipitation asymmetry is explained more by storm motion. The main 
contributor to the general asymmetry pattern is from the moderate and heavy precipitation. The wavenumber 2-6 energy localizes the maximum heavy precipitation asymmetry.

The quantified wavenumber 1-6 asymmetry is also applied to differentiate between different intensity change categories and the asymmetry evolution of a rapidly intensifying storm. The precipitation asymmetry properties of rapid intensification (RI) and non-RI storms are examined. The dataset of 2,186 global tropical storms through category 2 hurricanes is divided by future 24-h intensity change and includes storms with at least moderately favorable environmental conditions. The normalized wavenumber 1-6 asymmetries, indicates quantitatively that the lower asymmetry of precipitation is most strongly correlated with future intensity change. The precipitation field of non-RI storms are more asymmetric than RI storms. The 595 sampled overpasses are classified into 14 categories in the timeline of an RI event from 48 hours before RI until RI ends. The decrease of normalized wavenumber 1-6 asymmetries in the inner core region of all four types of precipitation several hours before RI onset was quantitatively demonstrated to be critical for TC RI. 


\section{TABLE OF CONTENTS}

CHAPTER

PAGE

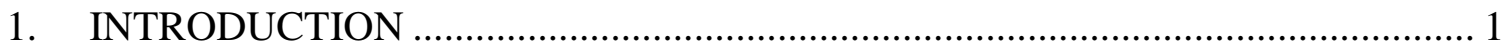

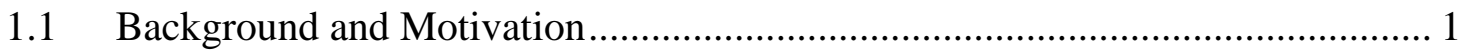

1.2 Tropical Cyclone Precipitation......................................................................... 3

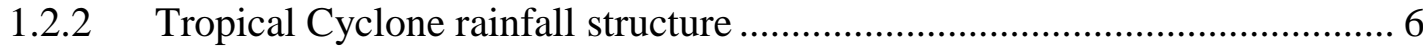

1.3 Tropical Cyclone Rainfall Asymmetry Quantification ......................................... 7

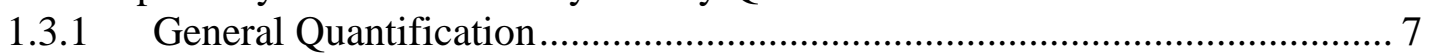

1.3.2 Quantification through Fourier Decomposition............................................ 9

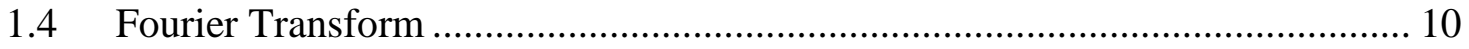

1.4.1 Discrete Fourier Transform.............................................................. 10

2. QUANTIFICATION OF PRECIPITATION ASYMMETRY OF TROPICAL CYCLONES USING 16-YR TRMM OBSERVATIONS........................................... 20

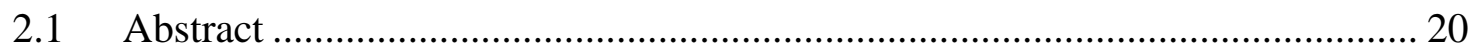

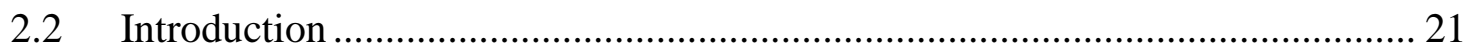

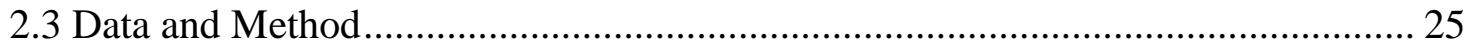

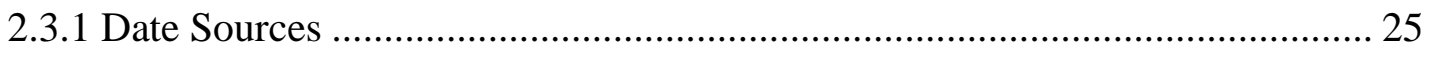

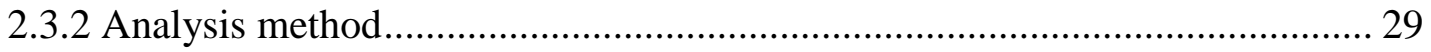

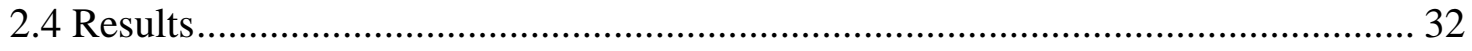

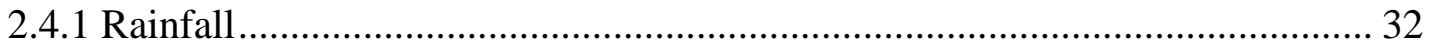

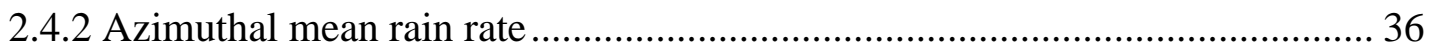

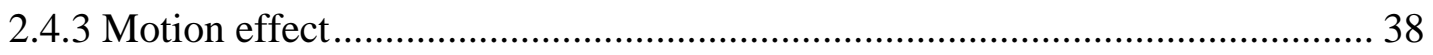

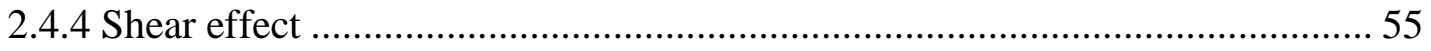

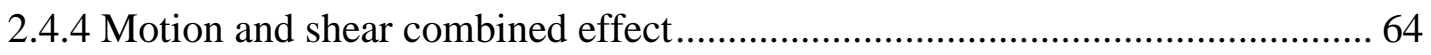

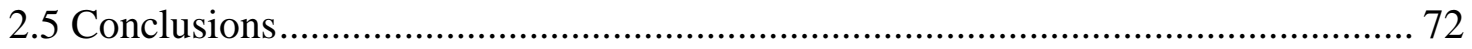

3. QUANTIFICATION OF SHEAR-RELATIVE PRECIPITATION ASYMMETRIES OF TROPICAL CYCLONES IN DIFFERENT INTENSITY

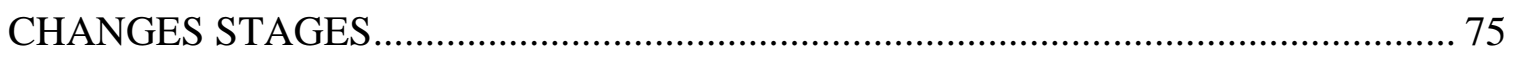

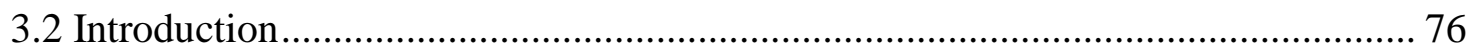

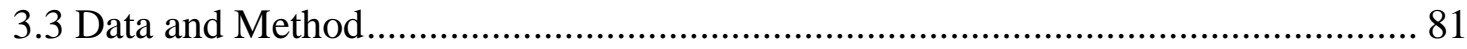

3.3.1 TRMM overpass selection .......................................................................... 81

3.3.2 Intensity change categories and RI event-based dataset ............................... 83

3.3.3 Definition of three kinds of precipitation...................................................... 86

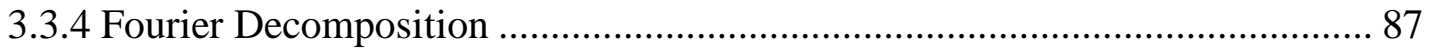

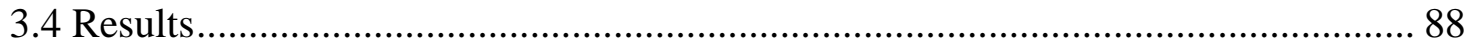

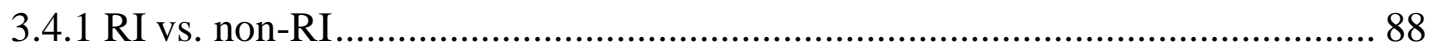

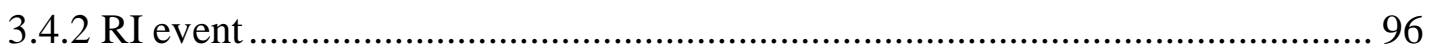

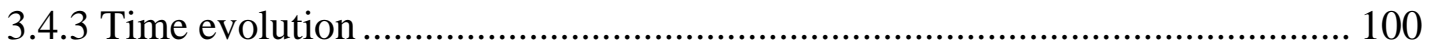

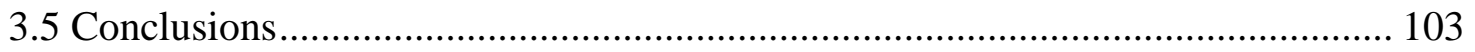




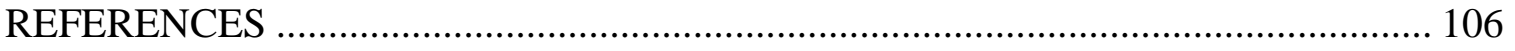

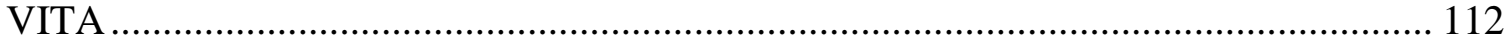




\section{LIST OF TABLES}

TABLE

PAGE

Table 1.1: Rain rate measurements time series. For illustration purpose, the data are

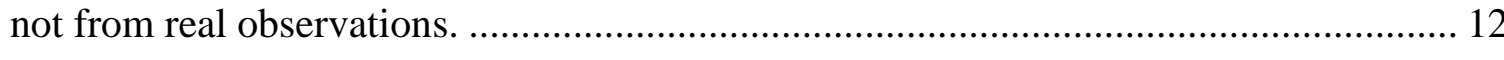

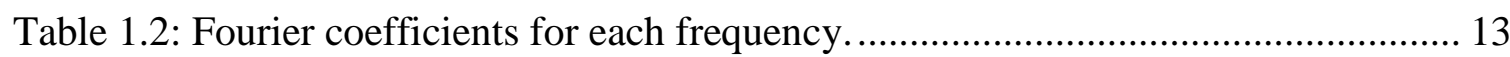

Table 2.1: Numbers of selected TMI overpasses for different intensity stages in different basins.

Table 2.2: Mean values of current TC intensity $\mathrm{V}_{\max }$, vertical wind shear magnitude, storm motion speed, and numbers (percentages) of selected TMI overpasses with shear same as, opposite to, to the left of, and to the right of motion for the total dataset, different oceanic basins, and different intensity stages. 28

Table 2.3: The percentage of total perturbation energy contributed by wavenumber (WN) 1, 2, 3, 4, 5, 6, 2-6 (2 through 6) and > 6 perturbations averaged over the annulus from 0 to $200 \mathrm{~km}$ and from 30 to $80 \mathrm{~km}$ from the TC center for total precipitation, light precipitation, moderate precipitation, and heavy precipitation, respectively.

Table 2.4: (for Fig. 3) The asymmetry index (AI) of the composite motion-relative (Row I) wavenumber 1 asymmetry ( $R_{1}$ ), (Row II) $R_{1} / R_{0}$, (Row III) $R_{1-6}$, (Row IV) $\mathrm{R}_{1-6} / \mathrm{R}_{0}$ of [four columns]: (a) total precipitation, (b) light precipitation, (c) moderate precipitation, and (d) heavy precipitation. The percentage in each pair of parentheses represents the percentage increase of the asymmetry index from $R_{1}$ to $\mathrm{R}_{1-6}$ or from $\mathrm{R}_{1} / \mathrm{R}_{0}$ to $\mathrm{R}_{1-6} / \mathrm{R}_{0}$

Table 2.5: (for Fig. 2.4 and 2.5) The asymmetry index of the composite motion-relative wavenumber 1 and wavenumber 1-6 asymmetry normalized by the azimuthal mean $\left(\mathrm{R}_{1} / \mathrm{R}_{0}\right.$ and $\left.\mathrm{R}_{1-6} / \mathrm{R}_{0}\right)$ of total precipitation for six basins. The percentage in each pair of parentheses represents the percentage increase of asymmetry index from $R_{1} / R_{0}$ to $R_{1-6} / R_{0}$.

Table 2.6: (for Fig. 6 and 7) The asymmetry index of the composite motion-relative $\mathrm{R}_{1} / \mathrm{R}_{0}$ and $\mathrm{R}_{1-6} / \mathrm{R}_{0}$ of total precipitation for (Row I) all TCs, (Row II) slow moving TCs, and (Row III) fast moving TCs of [three columns] (a) TS, (b) CAT1-2, (c) CAT3-5. basins. The percentage in each pair of parentheses represents the percentage increase of asymmetry index from $R_{1} / R_{0}$ to $R_{1-6} / R_{0}$

Table 2.7: (for Fig. 9 and 10) The asymmetry index of the composite shear-relative wavenumber 1 and wavenumber 1-6 asymmetry normalized by the azimuthal mean 
$\left(\mathrm{R}_{1} / \mathrm{R}_{0}\right.$ and $\left.\mathrm{R}_{1-6} / \mathrm{R}_{0}\right)$ of total precipitation for six basins. The percentage in each pair of parentheses represents the percentage increase of asymmetry index from $R_{1} / R_{0}$ to $\mathrm{R}_{1-6} / \mathrm{R}_{0}$.

Table 2.8 (for Fig. 11 and 12) The asymmetry index of the composite shear-relative $\mathrm{R}_{1} / \mathrm{R}_{0}$ and $\mathrm{R}_{1-6} / \mathrm{R}_{0}$ of total precipitation for (Row I) TS, (Row II) CAT 1-2, and (Row III) CAT 3-5 with [three columns] (a) low environment wind shear, (b) moderate environment wind shear, (c) high environment wind shear. The percentage in each pair of parentheses represents the percentage increase of asymmetry index from $R_{1} / R_{0}$ to $R_{1-6} / R_{0}$.

Table 2.9 (for Fig. 14 and 15) The asymmetry index of the composite shear-relative $R_{1} / R_{0}$ and $R_{1-6} / R_{0}$ of total precipitation with the shear direction (a) same as, (b) opposite to, (c) to the right of, and (d) to the left of the motion direction for (I) slow motion and low shear, (II) slow motion and moderate shear, (III) slow motion and high shear, (IV) fast motion and low shear, (V) fast motion and moderate shear, and (VI) fast motion and high shear conditions. The percentage in each pair of parentheses represents the percentage increase of asymmetry index from $R_{1} / R_{0}$ to $\mathrm{R}_{1-6} / \mathrm{R}_{0}$.

Table 3.1:The sample size of different intensity change categories 82

Table 3.2: The sample size in different TC intensity change categories for before RI, during RI and in the RI ending period.

Table 3.3: Mean values of maximum wind, Sea Surface Temperature (SST), Total Precipitable Water (TPW), vertical wind shear magnitude, storm motion speed, and the direction difference between motion and vertical wind shear in degree for different intensity change categories and total dataset.

Table 3.4: The asymmetry index of the composite shear-relative $R_{1-6} / R_{0}$ of total precipitation, light precipitation, moderate precipitation, and heavy precipitation of [four columns] weakening, neutral, slowly intensifying, and rapid intensifying TCs...... 91 
Figure 1.1: Location of initial genesis points of tropical cyclones for a 20-year period (Gray, 1979).

Figure 1.2 Latitude variations of sea surface temperatures (SST) in the various TC development regions during the warmest summer month (Gray, 1967). 5

Figure 1.3: (a-c) Superposition of sine and cosine waves that recreate (d) the original timeseries.

Figure 1.4: Hurricane Earl on Aug. 29 $9^{\text {th }}, 2010$ at 13:04 UTC. (a) the precipitation fields, (b) the mean precipitation, (c) wavenumber 1 asymmetry, (d) wavenumber 2 asymmetry, (e) wavenumber 3 asymmetry, and (f) wavenumber 4 asymmetry for precipitation fields. The black arrow is the vertical wind shear direction.

Figure 1.5: Schematic view of the scan geometries of the three TRMM primary rainfall sensors: TRMM Microwave Imager (TMI), Precipitation Radar (PR), and Visible and infrared scanner (VIRS) (Kummerow et al.1998).

Figure 2.1: The percentage of total perturbation energy contributed by wavenumber (WN) 1, 2, 3, 4, 5, 6, and 1-6 (1 through 6) as a function of radial distance for (a) total precipitation, (b) light precipitation, (c) moderate precipitation, and (d) heavy precipitation.

Figure 2.2: Mean rain rates $\left(\mathrm{R}_{0}\right)$ for (a) three different intensity stages (b) six oceanic basins, and (c) three precipitation types.

Figure 2.3: The composite motion-relative (Row I) wavenumber 1 asymmetry $\left(\mathrm{R}_{1}\right)$, (Row II) $\mathrm{R}_{1} / \mathrm{R}_{0}$, (Row III) $\mathrm{R}_{1-6}$, (Row IV) $\mathrm{R}_{1-6} / \mathrm{R}_{0}$ of [four columns]: (a) total precipitation, (b) light precipitation, (c) moderate precipitation, and (d) heavy precipitation. The white arrow (pointing upward) represents the orientation of motion vector. The color scale represents average perturbation of the sum of the wavenumber 1 or 1-6 asymmetry components. Dotted range rings represent the $50-, 100-, 150-$, and $200-\mathrm{km}$ radii.

Figure 2.4: The composite motion-relative wavenumber 1 asymmetry normalized by the azimuthal mean $\left(\mathrm{R}_{1} / \mathrm{R}_{0}\right)$ of total precipitation for six basins. The white arrow (pointing upward) represents the orientation of motion vector. The color scale represents average perturbation of the sum of the wavenumber 1 or 1-6 asymmetry components. Dotted range rings represent the 50-, 100-, 150-, and 200-km radii. 44

Figure 2.5: Same as FIG. 2.4, but for $\mathrm{R}_{1-6} / \mathrm{R}_{0}$. 46 
Figure 2.6: The composite motion-relative $\mathrm{R}_{1} / \mathrm{R}_{0}$ of total precipitation for (Row I) all TCs, (Row II) slow moving TCs, and (Row III) fast moving TCs of [three columns] (a) TS, (b) CAT1-2, (c) CAT3-5. The white arrow (pointing upward) represents the orientation of motion vector. The color scale represents average perturbation of the sum of the wavenumber 1 or 1-6 asymmetry components. Dotted range rings represent the 50-, 100-, 150-, and 200-km radii

Figure 2.7: Same as FIG. 2.6, but for $\mathrm{R}_{1-6} / \mathrm{R}_{0}$.

Figure 2.8: The motion-relative wavenumber-based Asymmetry Index (integrated from 0 to $200 \mathrm{~km}$ radius) of total precipitation as a function of motion speed for (a) three different intensity stages with solid line for WN 1-6 and dot line for WN 1, and (b) six different basins with solid line for WN1-6 and dot line for WN 1 .

Figure 2.9: The composite shear-relative wavenumber 1 asymmetry normalized by the azimuthal mean $\left(\mathrm{R}_{1} / \mathrm{R}_{0}\right)$ of total precipitation for six basins. The black arrow (pointing upward) represents the orientation of motion vector. The color scale represents average perturbation of the sum of the wavenumber 1 asymmetry components. Dotted range rings represent the 50-, 100-, 150-, and 200-km radii. 56

Figure 2.10: Same as FIG. 2.9, but for $\mathrm{R}_{1-6} / \mathrm{R}_{0}$. 57

Figure 2.11: The composite shear-relative $\mathrm{R}_{1} / \mathrm{R}_{0}$ of total precipitation for (Row I) TS, (Row II) CAT 1-2, and (Row III) CAT 3-5 with [three columns] (a) low environment wind shear, (b) moderate environment wind shear, (c) high environment wind shear. The black arrow (pointing upward) represents the orientation of motion vector. The color scale represents average perturbation of the sum of the wavenumber 1 or 1-6 asymmetry components. Dotted range rings represent the 50-, 100-, 150-, and $200-\mathrm{km}$ radii

Figure 2.12: Same as FIG. 2.11, but for $\mathrm{R}_{1-6} / \mathrm{R}_{0}$

Figure 2.13: Same as FIG. 2.8, but for shear-relative wavenumber-based Asymmetry Index (integrated from 0 to $200 \mathrm{~km}$ radius) of total precipitation as a function of shear magnitude.

Figure 2.14: The composite shear-relative $\mathrm{R}_{1} / \mathrm{R}_{0}$ of total precipitation with the shear direction (a) same as, (b) opposite to, (c) to the right of, and (d) to the left of the motion direction for (I) slow motion and low shear, (II) slow motion and moderate shear, (III) slow motion and high shear, (IV) fast motion and low shear, (V) fast motion and moderate shear, and (VI) fast motion and high shear conditions. The black arrow (pointing upward) represents the orientation of vertical wind shear vector. The white arrow represents the mean orientation of motion vector. The color scale represents average perturbation of the sum of the wavenumber 1-6 asymmetry components. Dotted range rings represent the 50-, 100-, 150-, and 200-km radii. 
Figure 2.15: Same as FIG. 2.14, but for the composite shear-relative $\mathrm{R}_{1-6} / \mathrm{R}_{0}$ of total precipitation.

Figure 2.16: The composite motion-relative sum of $\mathrm{R}_{1} / \mathrm{R}_{0}$ of total precipitation with the shear direction (a) same as, (b) opposite to, (c) to the right of, and (d) to the left of the motion direction for (I) slow motion and low shear, (II) slow motion and moderate shear, (III) slow motion and high shear, (IV) fast motion and low shear, (V) fast motion and moderate shear, and (VI) fast motion and high shear conditions. The black arrow represents the orientation of vertical wind shear vector. The white arrow (pointing upward) represents the mean orientation of motion vector. The color scale represents the average perturbation of the sum of the wavenumber 1-6 asymmetry components. Dotted range rings represent the 50-, 100-, 150-, and 200-km radii.....

Figure 2.17: Same as FIG. 2.16, but for the composite motion-relative $\mathrm{R}_{1-6} / \mathrm{R}_{0}$ of total precipitation.

Figure 3.1: Composite shear-relative $\mathrm{R}_{1-6} / \mathrm{R}_{0}$ for total precipitation for (a) W, (b) $\mathrm{N}$, (c) SI, (d) RI

Figure 3.2: Composite shear-relative $\mathrm{R}_{1-6} / \mathrm{R}_{0}$ for light precipitation for (a) $\mathrm{W}$, (b) $\mathrm{N}$, (c) SI, (d) RI.

Figure 3.3: Composite shear-relative $\mathrm{R}_{1-6} / \mathrm{R}_{0}$ for moderate precipitation for (a) $\mathrm{W}$, (b) N, (c) SI, (d) RI

Figure 3.4: Composite shear-relative $\mathrm{R}_{1-6} / \mathrm{R}_{0}$ for heavy precipitation for (a) $\mathrm{W}$, (b) $\mathrm{N}$, (c) SI, (d) RI

Figure 3.5: Composite shear-relative $\mathrm{R}_{1-6} / \mathrm{R}_{0}$ for (I) total precipitation, (II) light precipitation, (III) moderate precipitation, and (IV) heavy precipitation. (a) 12-24 h before RI onset, (b) 0-12 h before RI onset, (c) RI initial, (d) RI continuing, and (e) 12-24 h before RI ends. Dotted range rings represent the 25-, 50-, 75-, and 100-km radii.

Figure 3.6: Hovmöller diagrams of azimuthal wavenumber-based Asymmetry Index from 0 to $200 \mathrm{~km}$ for (a) total precipitation, (b) light precipitation, (c) moderate precipitation, and (d) heavy precipitation. Dashed lines from left to right represent RI onset and $24 \mathrm{~h}$ before RI ends. 
List of Abbreviations and Acronyms

AI

ATL

CAT 1-2

CAT 3-5

EPA

ENSO

GFDL

MJO

$\mathrm{NH}$

NIO

NWP

PR

PF

R-CLIPER

RI

RI CON

RI INI

RMW

$\mathrm{SH}$

SI

$\mathrm{SIO}$
Asymmetry Index

Atlantic

Category 1-2 hurricanes

Category 3-5 hurricanes

East central Pacific

El Niño-Southern Oscillation

Geophysical Fluid Dynamics Laboratory

Madden-Julian Oscillation

Northern Hemisphere

North Indian Ocean

Northwest Pacific

Precipitation Radar

Precipitation Feature

refined Rainfall Climatology and Persistence

Rapidly Intensification

Rapidly Intensification continuing

Rapidly Intensification initial

Radius of maximum wind

Southern Hemisphere

Slowly Intensification

South Indian Ocean 
SPA

TC

TCPF

TMI

TRMM

TS

WN
South Pacific

Tropical Cyclone

Tropical Cyclone Precipitation Feature

TRMM Microwave Imager

Tropical Rainfall Measuring Mission

Tropical Storm

Wavenumber 


\section{INTRODUCTION}

\subsection{Background and Motivation}

Severe tropical cyclones (TCs) are among the most destructive of all natural disasters. Its capability of obliterating coastal regions threatens life. As defined by the National Hurricane Center, "A tropical cyclone is a rotating, organized system of clouds and thunderstorms that originate over tropical or subtropical water and has a low-level circulation." A cause of death directly attributable to Atlantic TCs between 1963 and 2012 for 2,325 people in the United States were identified in Rappaport (2014). Roughly 90\% of the deaths occurred in water-related incidents. Storm surge was the number one cause (49\%) of the deaths. Rain-related incidents induced by TCs accounted for $27 \%$ of the deaths. Although the total number of fatalities from storm surge is about double the total attributed to rain, about $50 \%$ of the deadly U.S. TCs had a death in flooding from rain. Only about $10 \%$ of the deadly U.S. TCs had a death in storm surge. Rain-related incidents causing death are the most frequent catastrophe. The quantitative measurement of rainfall in TCs is crucial in depicting TC rainfall structure and understanding the risk from TC rainfall.

For the past few decades, despite large improvement in the track forecast of tropical cyclones (TCs), there are few improvements in the intensity forecast (Cangialosi and Franklin 2012). Intensity forecasting is challenging because the processes important in intensity change occur within and between scales of many orders of magnitude, including environmental, vortex, convective, turbulent, and microscales (Marks and Shay 1998). Other than intensity forecast, predictability limit for TC precipitation also has long been 
recognized (Elsberry 2002). It has been challenging to improve 72-h quantitative precipitation forecasts (QPF) for TCs, consequently day-3 forecasts for inland flooding. By comparing the skills of Geophysical Fluid Dynamics Laboratory (GFDL) model and a refined Rainfall Climatology and Persistence (R-CLIPER) model (Marks et al. 2002) rainfall forecasts, Tuleya et al. (2007) found the GFDL model exhibited a higher pattern of correlation versus daily rain gauge data than did R-CLIPER, but still only explained $\sim 30 \%$ of the spatial variance. Lonfat et al. (2007) introduced Parametric Hurricane Rainfall Model (PHRaM) integrating spatial rainfall asymmetries from shear effects to R-CLIPER model. The PHRaM fails at producing strong rainfall asymmetries caused by vertical shear. It is because the model uses the average statistical satellite observation. However, it is also likely a result of only incorporating first and second order (without higher orders) rainfall asymmetry using Fourier decomposition in PHRaM. The detailed quantitative information of TC precipitation derived from long-term TC rainfall statistics with refined method is still needed to validate and enhance the forecast accuracy of numerical models and statistical techniques.

Radar and satellite images of TCs have clearly shown that during the lifetime of a $\mathrm{TC}$, the precipitation pattern is usually asymmetric, dominated by low-wavenumber components. It has been demonstrated that the asymmetric distribution of TC precipitation is influenced by the TC motion and environmental vertical wind shear (Chan et al. 2004; Lonfat et al 2004; Chen et al. 2006). The rainfall asymmetry was quantified by the firstorder Fourier decomposition relative to storm motion (Lonfat et a. 2004) and relative to environmental vertical shear (Chen et al. 2006). Furthermore Chen et al. (2006) showed the juxtaposition of storm motion and relative magnitude of environmental shear vectors 
on TC rainfall asymmetry. However, when considering the juxtaposition effects on rainfall asymmetry, the relative magnitude of storm motion was not considered. The clear collective effects of motion and shear need a further study. On the other hand, the degree of precipitation asymmetries is also related to the storm Rapid Intensification (RI), as RI storm usually exhibits symmetric precipitation and convection pattern (Kieper and Jiang 2012; Zagrodnik and Jiang 2014; Tao and Jiang 2015).

Therefore, this dissertation explains the combined effects on TC rainfall asymmetry, and provide the relationship between rainfall asymmetry and storm Rapid Intensification. Before discussing the results of the study, the current knowledge of TC rainfall structure, intensification, and technique used in this study is described in the following sections including 1) TC formation and intensification, 2) TC rainfall structure 3) TC rainfall asymmetry quantification 4) Fourier Transform, and 5) Rainfall retrieval algorithm.

\subsection{Tropical Cyclone Precipitation}

\subsubsection{Tropical Cyclone Life Cycle}

Many theoretical works (Ooyama 1969, 1982; Montgomery 1993; Gray 1998) have debated the different mechanisms of cyclone genesis. The complex processes of cyclone genesis are also involved with Monsoon, Madden-Julian Oscillation (MJO), and El NiñoSouthern Oscillation (ENSO) (Simpson 1997; Camargo et al. 2007; Camargo et al. 2009). However, several necessary conditions for cyclone-genesis are widely accepted. Tropical Cyclones generally form in moist unstable environments with tropical disturbances and warm sea surface temperatures presenting. Gray $(1968 ; 1979)$ investigated the 
climatological aspects of the season frequency of TC formation, which are closely related to the following factors:

1. The Coriolis parameter $(f)$

2. Ocean thermal energy, manifest as ocean temperature greater than $26.5^{\circ} \mathrm{C}$ to a depth of 60 meters

3. The difference in equivalent potential temperature between the surface and 500 $\mathrm{mb}\left(\partial \theta_{e} / \partial p\right)$

4. Middle tropospheric relative humidity

5. Inverse of the tropospheric vertical wind shear

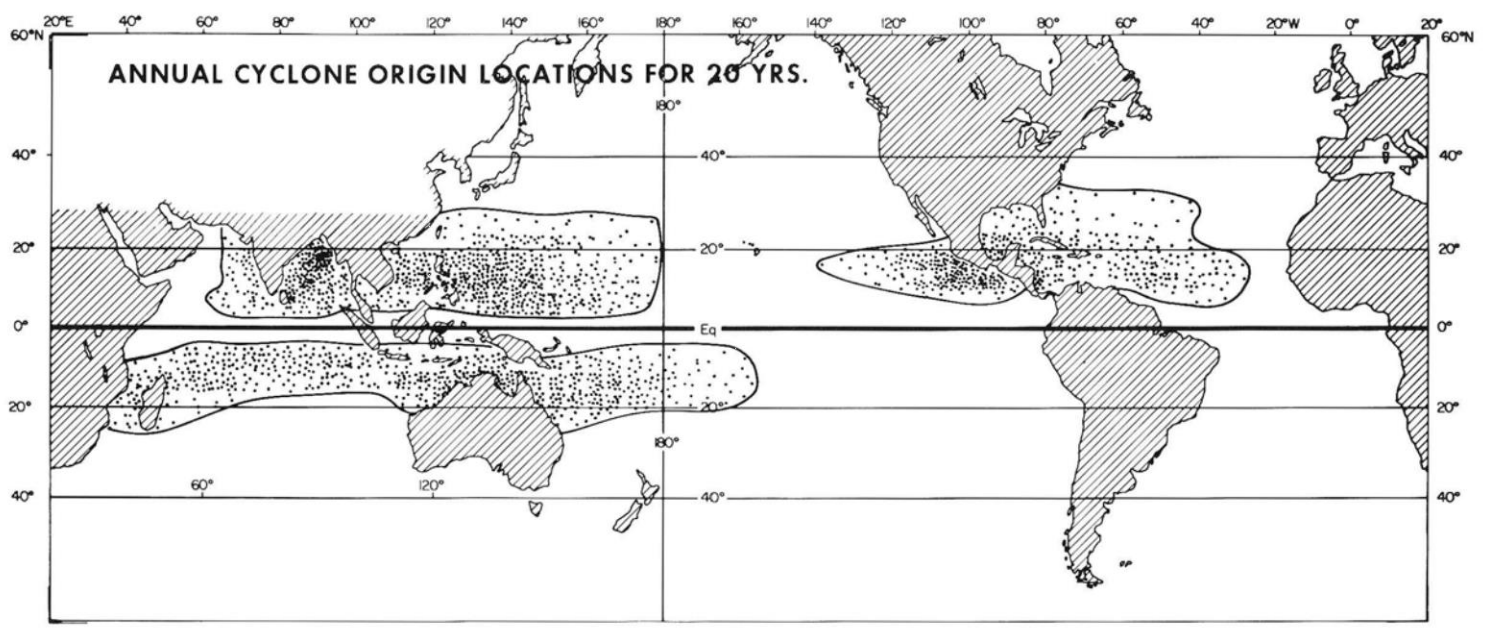

Figure 1.1: Location of initial genesis points of tropical cyclones for a 20 -year period (Gray, 1979).

The Coriolis parameter is one of the factors that provide necessary conditions for forming TCs that produces planetary vorticity. The absolute vorticity in a hurricane has a value $\sim 2 \times 10^{-4} s^{-1}$ (Gray and Shea, 1973). So TCs barely form between $-5-5^{\circ}$ of the Equator. The majority forms in the zone between $\pm 10^{\circ}- \pm 20^{\circ}$ (Fig. 1.1). Palmén (1948) mentioned that hurricanes can be formed only in the oceanic regions outside the vicinity of 
the Equator where the sea surface temperature is above the critical temperature of 26-27 ${ }^{\circ} \mathrm{C}$.

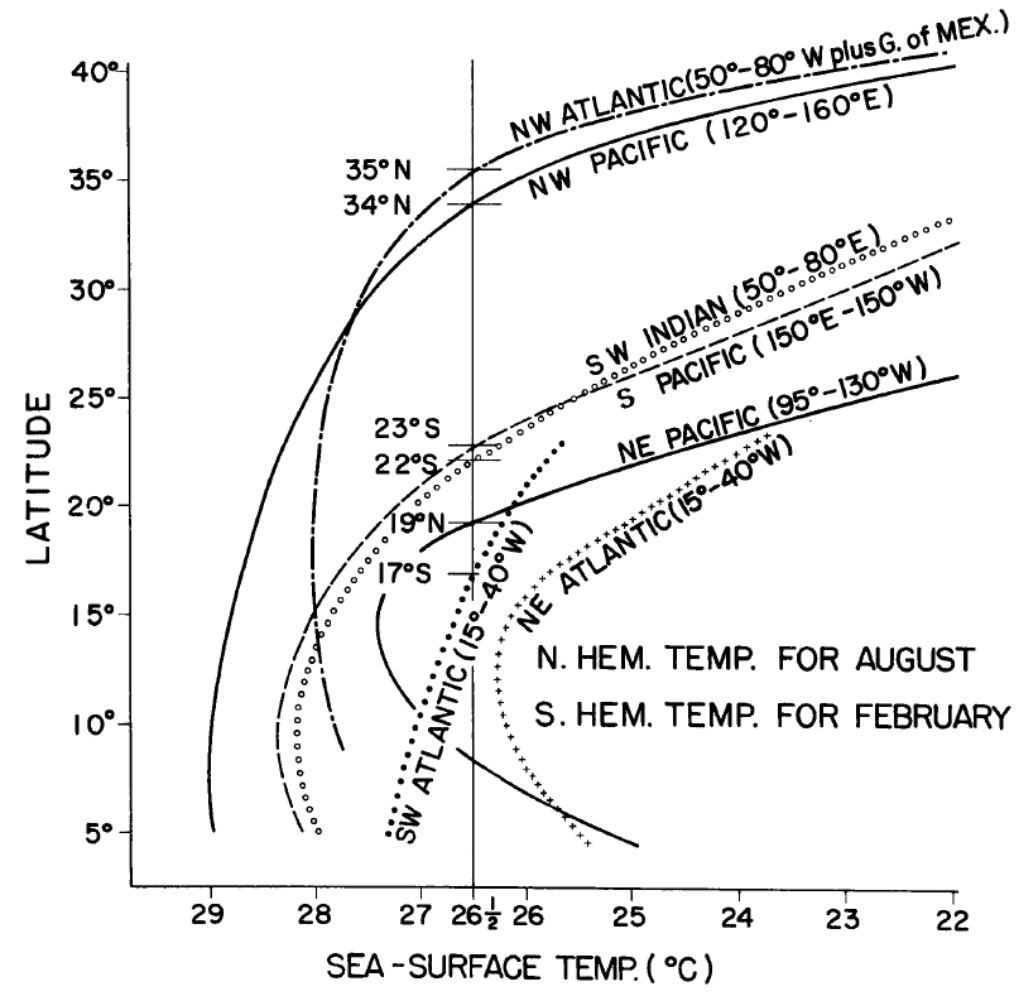

Figure 1.2 Latitude variations of sea surface temperatures (SST) in the various TC development regions during the warmest summer month (Gray, 1967).

Figure 1.2 shows the sea-surface temperatures regional distributions during the warmest summer month. The favorable regions are mostly in lower latitudes of the Atlantic, northwest Pacific, southwest Indian Ocean, Southern Pacific, and a small region in the northeast Pacific Ocean. Conditional instability must exist as a necessary condition for the formation of cumulus convection. As convective clouds originate in the boundary layer are mixed with the mid-troposphere air, the air must have high humidity to maintain the convection. In a tropical cyclone, the mid-troposphere is usually a convergence zone, so the humidity is normally high. Disturbance would develop when the vertical wind shear is 
small. Under low wind shear, the advection of heat and moisture is small, which may increase the moisture and temperature throughout the disturbance. When the shear is large, the high temperature and moisture are advected away causing unfavorable conditions. Tropical cyclones will develop under these favorable environmental and dynamical conditions. However, they begin to lose their tropical characteristics when they move out of the warm environment, moist air, move over land, or move to high shear regions. Especially when TCs move to higher latitudes, as the release of latent heat is diminished, the upper-level divergence weakens, the center pressure will rise so that TCs will decay. If a TC moves over land, it weakens. As the storm leaves the ocean, the evaporation will be reduced. The source of convection is terminated. The process of condensation and latent heating releasing are diminished. Also, the land is usually cooler and has higher roughness.

\subsubsection{Tropical Cyclone rainfall structure}

The dominant cloud type in $\mathrm{TC}$ is the cumulonimbus. The reason that there are more convective clouds in TCs than stable and layered cloud is because potential instability leads the low-level convergence. Convective clouds cover up to $50 \%$ of the area within the 100-km radius around storm center (Frank 1977). The strong updrafts in the clouds produce a cirrus overcast at an altitude of $10-15 \mathrm{~km}$. The eye of the storm would be clear with a strong subsidence that extends to low levels. With the combined effects of evaporation of sea water, moist air, and strong horizontal convergence, TCs usually produce heavy rainfall. 
Using a three-dimensional Tropical Rainfall Measurement Mission (TRMM) Precipitation Radar (PR) dataset, Hence and Houze (2011) found that the TC eyewall has a deep and intense structure, which is related to the secondary circulation. In the eyewall region, extremely strong convection often presents, which are likely caused by buoyant convective towers superimposed upon the mean circulation. In the eyewall region, relative to vertical wind shear direction, it was shown that the primary precipitation area is located at the front-left region. The convective generation of precipitation is in the front-right region relative to wind shear. In the rainband area, the right-of-shear quadrants contain newer convection while in the left-of-shear quadrants there is mainly stratiform precipitation (Hence and Houze 2012). Jiang et al. (2012) demonstrated the prominent difference of characteristics of precipitation between eyewall and rainband is that strong convective signatures occur generally more often in the eyewall region, less in the rainband region.

\subsection{Tropical Cyclone Rainfall Asymmetry Quantification}

\subsubsection{General Quantification}

Many previous studies had attempted to use the quantitative information to describe the distribution of TC asymmetric rainfall structure. Cecil (2007) analyzed the rainfall asymmetry relative to environmental vertical wind shear with varying magnitudes using the microwave rain rates data from Remote Sensing Systems (RSS). The rain rate data were from Special Sensor Microwave Imager (SSM/I) and Tropical Rainfall Measuring Mission 
(TRMM) Microwave Imager (TMI) measurements. The TC rainfall asymmetry was represented into four quadrants $(\mathrm{NW}, \mathrm{NE}, \mathrm{SE}$, and $\mathrm{SW})$. It was shown that in the inner core (radius less than $100 \mathrm{~km}$ ), under strong shear downshear-left region had rain rates twice as large as other quadrants. In the consecutive study (Wingo and Cecil 2010), the version 4 RSS data were used to analyze the global rainfall distributions in TCs. The RSS rain rate data with a resolution of $0.25^{\circ} \times 0.25^{\circ}$ grid were degraded into a polar grid of $50-\mathrm{km}$ radial and $45^{\circ}$ azimuthal resolutions. Their results showed the relationships between the TC intensity, the geographical location, the shear magnitude, the rainfall magnitude and the rainfall asymmetry relative to the shear direction. It indicates the precipitation is displaced downshear to the left (right for Southern Hemisphere) of shear vector.

Not only in terms of rain rates, but using lightning data as a proxy of convection, Corbosiero and Molinari (2003) analyzed all named storms from 1985 to 1999 in the Atlantic basin observed by the National Lightning Detection Network (NLDN). The relative importance between storm motion and vertical wind shear were demonstrated. It was shown that the lightning-based convective asymmetries has a maximum convection in the front quadrant of the TC inner core. The maximum convection in the outer band region is right of motion. The maxima of convective asymmetries are mainly downshear left in the inner core and downshear right in the outer bands. With the same dataset, Corbosiero and Molinari (2002) evaluated the effects from vertical shear on convective asymmetries. It was shown that an asymmetric convection preference of downshear left occurring in the storm core and a strong preference of downshear right in the outer rain bands. In their study, convective asymmetries were examined by comparing the number of lightning counts in 
different shear-relative quadrants and showing the composite of flash locations. It was argued that the shear effect has been shown to have more importance over the storm motion on convection distribution (Corbosiero and Molinari 2003).

\subsubsection{Quantification through Fourier Decomposition}

To illustrate how storm motion affects TC rainfall asymmetry, using three years of Tropical Rainfall Measuring Mission (TRMM) microwave imager (TMI) surface rainfall estimates (Kummerow et al. 1996), using Fourier Transform, Lonfat et al. (2004) decomposed the rain rates of $2,121 \mathrm{TC}$ instantaneous overpasses to quantify the precipitation asymmetry relative to storm motion. They demonstrated that TC wavenumber-1 rainfall asymmetry varies among different basins, but in general the maximum asymmetry is located in the front quadrants relative to storm motion. Tropical storm (TS) shows more asymmetric precipitation than Category 1 to 2 (CAT 1-2) and Category 3 to 5 (CAT 3-5). Their results also indicate that for TS, the maximum asymmetry is located at front-left region, while the Category 3 to 5 storms have the maximum asymmetry located at front-right quadrant. By analyzing the same rainfall dataset from TRMM TMI as Lonfat et al. (2004), Chen et al. (2006) demonstrated the wavenumber-1 rainfall asymmetries of TC relative to environmental vertical shear and compared the shear-relative results to motion-relative results. Their results suggest that in the inner core region shear is dominant and downshear left region is favored by the precipitation asymmetries. When in the low shear environment, the storm translation speed plays an 
important role on the outer core rainfall asymmetry distribution; however, the inner core asymmetry is still controlled by shear. Ueno (2007) evaluated the rainfall asymmetry by showing the number of cases of the highest wavenumber-1 rain rate in each octant relative to vertical wind shear direction using Japan Meteorological Agency Radar network data and TMI and Advanced Microwave Scanning Radiometer - Earth Observing System (AMSR-E) data of Typhoon Chaba and Typhoon Tokage in 2004. The results showed that

maximum TC rainfall in the inner core tend to occur on the downshear to downshear-left side. In the low latitudes, the majority of storm motion directions are to the right of shear.

\section{$1.4 \quad$ Fourier Transform}

\subsubsection{Discrete Fourier Transform}

In Fourier analysis, any continuous function can be described by an infinite Fourier series, namely the sum of an infinite number of sine and cosine terms. In the case of a discrete timeseries with a finite number of points, it is required to have only a finite number of sine and cosine terms to fit our points exactly.

Using Euler's notation $[\exp (i x)=\cos (x)+\sin (x)$, where $i$ is the square root of -1$]$, the discrete Fourier series representation of $A(k)$ can be written as:

$$
\text { Inverse Transform: } \quad A(k)=\sum_{n=0}^{N-1} F_{A}(n) e^{i 2 \pi n k / N}
$$


where $n$ is the frequency, and $F_{A}(n)$ is the discrete Fourier transform. A time series with $N$ data points (indexed from $k=0$ through $N$-1) needs no more than $N$ different frequencies to describe it.

There are a number of ways to describe frequency:

$n=$ number of cycles (per time period $P$ ),

$\tilde{n}=$ cycles per second $=n / P$,

$f=$ radians per second $=2 \pi n / P=2 \pi n /(N \Delta t)$.

A frequency of zero $(n=0)$ denotes a mean value. The fundamental frequency, where $n=1$ means that exactly on wave fills the whole-time period, $P$. High frequencies correspond to harmonics of the fundamental frequency. For example, $n=5$ means that exactly 5 waves fill the period $P$.

The value of $F_{A}(n)$ is a complex number, where the real part represents the amplitude of the cosine waves and the imaginary part is the sine wave amplitude. It is a function of frequency because the waves of different frequencies must be multiplied by different amplitudes to reconstruct the original time series. If the original time series $A(k)$ is known, then these coefficients can be found from:

$$
\text { Forward Transform: } \quad F_{A}(n)=\sum_{=0}^{N-1}\left[\frac{A(k)}{N}\right] e^{-i 2 \pi n k / N}
$$


The equations of (1.1) and (1.2) are called Fourier transform pairs. The second equation performs the forward transform, creating a representation of the signal in phase space (or frequency). This process of (1.2) is also known as Fourier decomposition. The first equation performs the inverse transform, converting from frequencies back into physical space.

Given the following 8 data points of rain rate, $r$, as a function of time in Table 1.4.1, below is an example of discrete Fourier transform. In this timeseries, the number of data points $N=8$, and the time interval $\Delta t=15 \mathrm{~min}$. Thus, the total period is $P=N \Delta \mathrm{t}=2 \mathrm{~h}$.

Table 1.1: Rain rate measurements time series. For illustration purpose, the data are not from real observations.

\begin{tabular}{c|cccccccc}
\hline \hline $\begin{array}{c}\text { Index }(\mathrm{k}) \\
\text { Time }\end{array}$ & 0 & 1 & 2 & 3 & 4 & 5 & 6 & 7 \\
$(\mathrm{UTC})$ & 1800 & 1815 & 1830 & 1845 & 1900 & 1915 & 1930 & 1945 \\
$\mathrm{r}(\mathrm{mm} / \mathrm{hr})$ & 5 & 7 & 7 & 9 & 12 & 6 & 3 & 2 \\
\hline \hline
\end{tabular}

Using the Euler's formula, (1.4.1b) can be written as:

$$
F_{A}(n)=\frac{1}{N} \sum_{k=0}^{N-1} A(k) \cos \left(\frac{2 \pi n k}{N}\right)-\frac{i}{N} \sum_{k=0}^{N-1} A(k) \sin \left(\frac{2 \pi n k}{N}\right)
$$

As an example, for $n=0$, all of the cosines of zero are unity and all of the sines are zero. This leaves:

$$
{ }_{A}(0)=\frac{1}{N} \sum_{k=0}^{N-1} A(k)
$$


which is the mean of A. For the 8 data points: $F_{r}(0)=7.0-0.0 i$. For $n=1, F_{r}(1)=0.28$ 1.03i. The coefficients are shown in table 1.4.2. The timeseries have been transformed into frequency domain.

Table 1.2: Fourier coefficients for each frequency.

\begin{tabular}{c|cccccccc}
\hline \hline$n$ & 0 & 1 & 2 & 3 & 4 & 5 & 6 & 7 \\
\hline & & $0.28-$ & & $0.78-$ & & $0.78+$ & & $0.28+$ \\
$F_{q}(n)$ & 7 & $1.03 \mathrm{i}$ & 0.5 & $0.03 \mathrm{i}$ & 1 & $0.03 \mathrm{i}$ & 0.5 & $1.03 \mathrm{i}$ \\
\hline \hline
\end{tabular}

To perform the inverse transform using (1.4.1a), the data can be transformed from frequency domain into time domain. After applied Euler's formula, the equation is:

$$
A(k)=\sum_{k=0}^{N-1} F_{\text {real part }}(n) \cos \left(\frac{2 \pi n k}{N}\right)-\sum_{k=0}^{N-1} F_{\text {imag. part }}(n) \sin \left(\frac{2 \pi n k}{N}\right)
$$

Figure 1.3 shows each individual wave multiplied by its appropriate amplitude. The reconstructed time series fits the eight original data points. In between these points, however, the sum oscillates in a manner that is not necessarily realistic, but which is irrelevant because it occurs below the discretization resolution specified by the original data. 
(a)

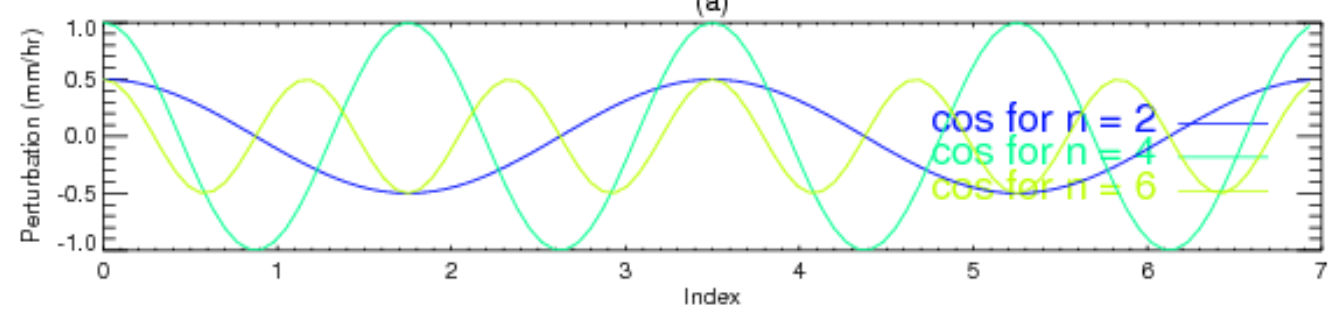

(b)

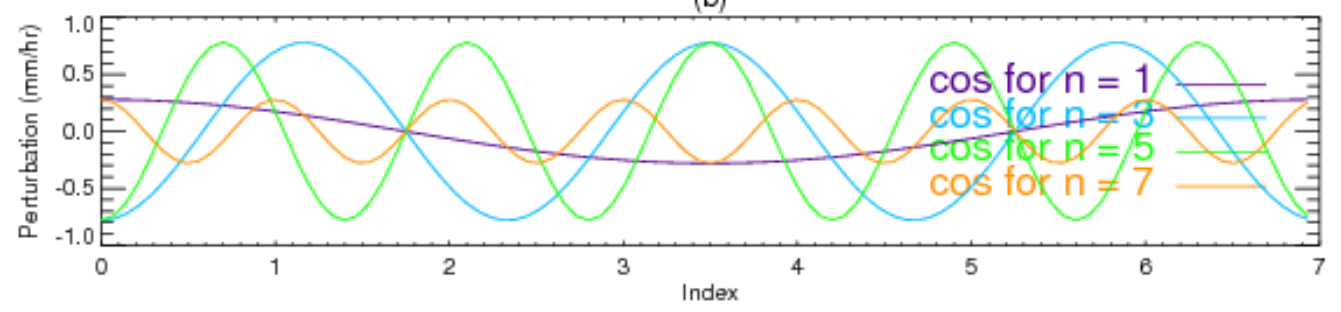

(c)

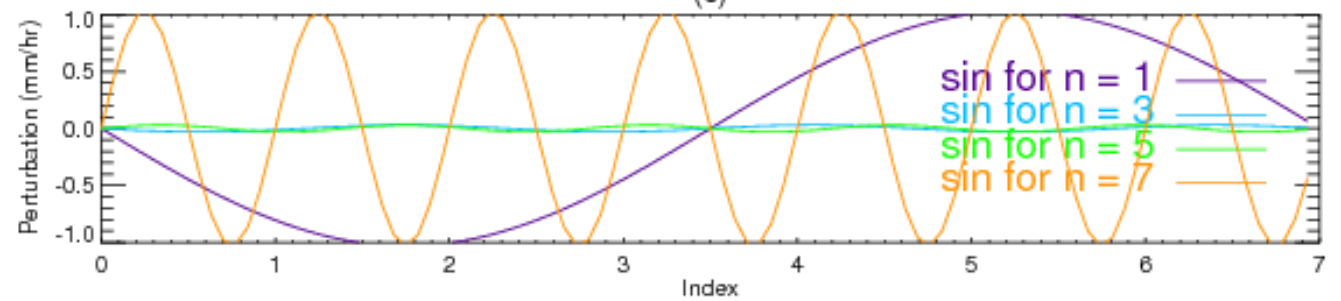

(d)

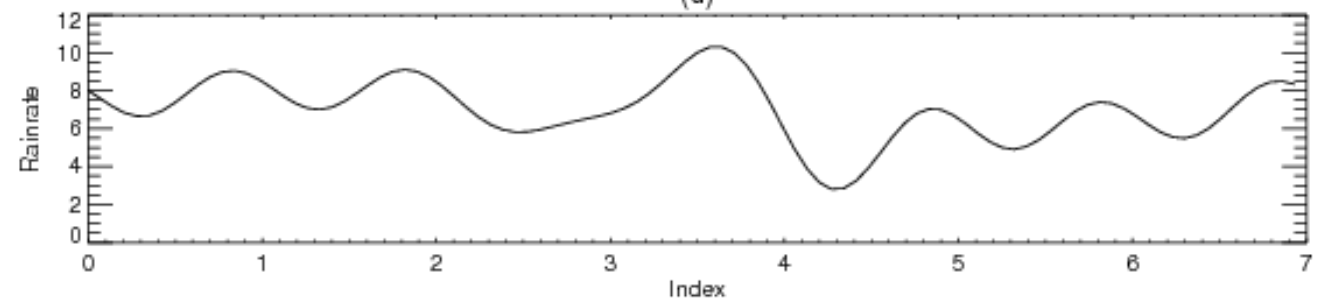

Figure 1.3: (a-c) Superposition of sine and cosine waves that recreate (d) the original timeseries.

A basic rule of discrete data analysis is that at least two data points are required per period or wavelength in order to resolve a wave. Since Fourier analysis involves splitting arbitrary signals into waves, the two data points requirement also holds for the arbitrary signals. For example, if there is a total of $\mathrm{N}$ data points, then the highest frequency that can be resolved in Fourier transform is $n_{f}=N / 2$, which is called Nyquist frequency. These requirements apply to measurements; namely, if a wave period as small as $0.1 \mathrm{~s}$ must be 
measured while flying in an aircraft, then the physical signal must be digitized at least once every $0.05 \mathrm{~s}$. Similarly, if a wavelength as small as $1 \mathrm{~m}$ must be measured, then the physical signal must be digitized at least once every $0.5 \mathrm{~m}$.

\subsubsection{Fast Fourier Transform}

The fast Fourier transform, or FFT, is a discrete Fourier transform that has been factored and restructured to take advantage of the binary computation processes of the digital computer. As a result, it produces the same output, and has the same limitations and requirements as the discrete transform. It can also be used for forward as well as inverse transforms. The FFT method is used throughout the current dissertation.

\subsubsection{Energy Spectrum}

In meteorological analysis, the contribution of the variance of a time series is associated with a particular frequency is often interesting, without regard to the precise phase of the waves. For turbulence, the original signal is not physically like waves at all, but it is still useful to break the signal into components of different frequencies that are associated with different eddy sizes. The square of the norm of the complex Fourier transform for a frequency $\mathrm{n}$ is:

$$
\left|F_{A}(n)\right|^{2}=\left[F_{\text {real part }}(n)\right]^{2}+\left[F_{\text {imag. } \operatorname{part}}(n)\right]^{2}
$$

When $\left|F_{A}(n)\right|^{2}$ is summed over frequencies $n=1$ to $N-1$, the result equals the total biased variance of the original time series: 


$$
\sigma_{A}^{2}=\frac{1}{N} \sum_{k=0}^{N-1}\left(A_{k}-\bar{A}\right)^{2}=\sum_{k=0}^{N-1}\left|F_{A}(n)\right|^{2}
$$

Thus, $\left|F_{A}(n)\right|^{2}$ can be interpreted as the portion of variance explained by waves of frequency $n$. Notice that the sum over frequencies does not include $n=0$, because $\left|F_{A}(0)\right|$ is the mean value and does not contribute any information about the variation of the signal about the mean.

\subsubsection{Fast Fourier Transform application on TC precipitation}

The analysis methods described in section 1.4 are illustrated using a TRMM observation of Hurricane Earl on Aug. $29^{\text {th }}, 2010$ at 13:04 UTC. Earl was located at $18^{\circ} \mathrm{N}$ and $58^{\circ} \mathrm{W}$. The minimum sea level pressure was near $983 \mathrm{mb}$ and the maximum sustained winds were about $65 \mathrm{~m} / \mathrm{s}$. Earl's rainfall displayed strong azimuthal variability in rain rates (Fig. 1.4a). Figure 1.4b-f shows the wavenumber 1 to 4 asymmetry spatial distribution within $200 \mathrm{~km}$ of Earl's center. To reconstruct all the wavenumber fields, the sum of all perturbations and mean will be equal to the original precipitation field. 

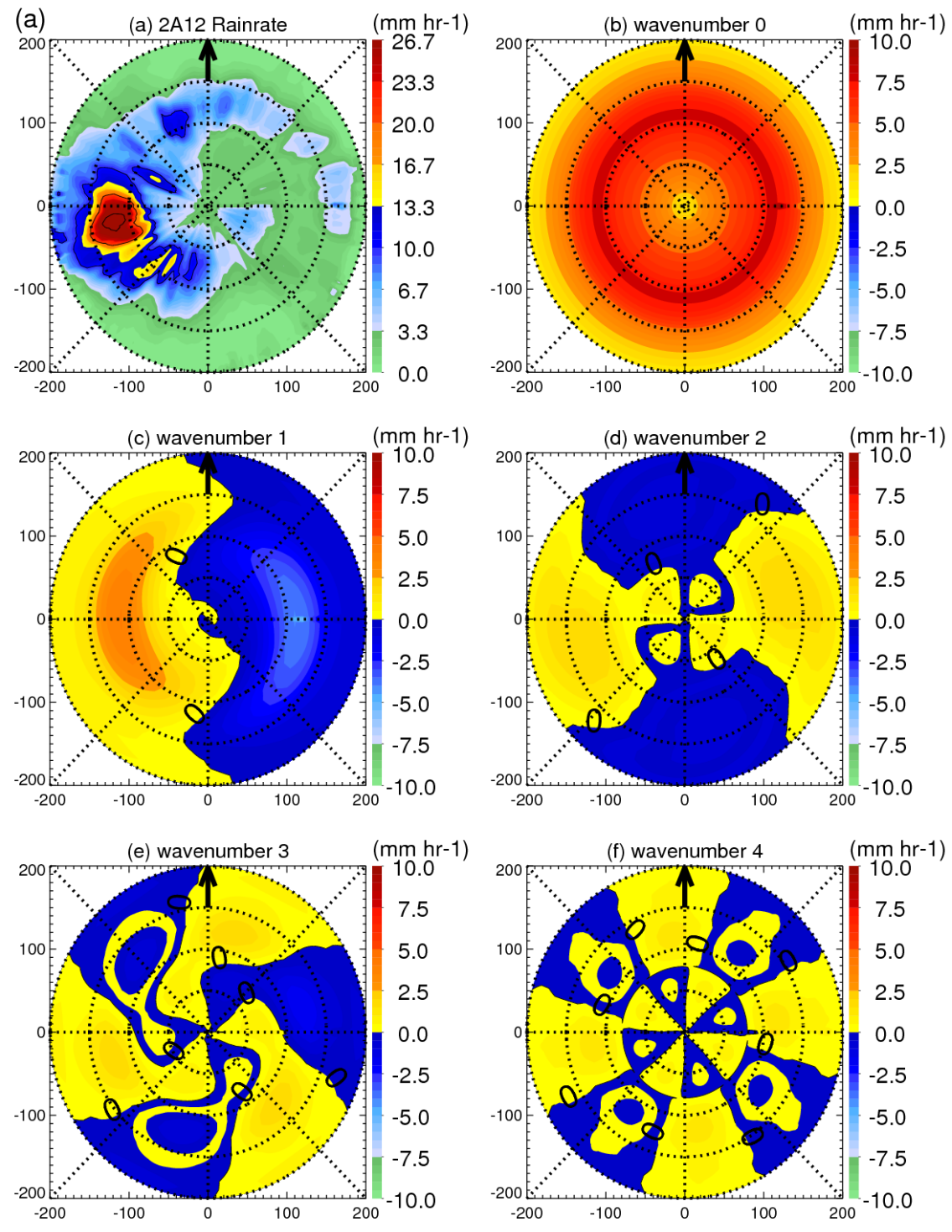

Figure 1.4: Hurricane Earl on Aug. 29 th 2010 at 13:04 UTC. (a) the precipitation fields, (b) the mean precipitation, (c) wavenumber 1 asymmetry, (d) wavenumber 2 asymmetry, (e) wavenumber 3 asymmetry, and (f) wavenumber 4 asymmetry for precipitation fields. The black arrow is the vertical wind shear direction. 


\subsection{Tropical Rainfall Measuring Mission}

\subsubsection{Microwave Imager}

The main observational data of this study are derived from the Tropical Rainfall Measurement Mission (TRMM) Microwave Imager (TMI) data. The TMI is a nine-channel passive microwave radiometer that uses the Special Sensor Microwave/Imager (SSM/I), which is the satellite of Defense Meteorological Satellite Program (DMSP). Tropical Rainfall Measuring Mission Imager added a pair of 10.7-GHz channels with horizontal and vertical polarizations and a frequency change of the water vapor channel from 22.235 to $21.3 \mathrm{GHz}$.

The TMI antenna is an offset parabola, with an aperture size of $61 \mathrm{~cm}$ and a focal length of $50.8 \mathrm{~cm}$. The incident angle is $52.8^{\circ}$ at the earth's surface. The TMI antenna rotates about a nadir axis at a constant speed of $31.6 \mathrm{rpm}$. The rotation draws a circle on the earth's surface. From the TRMM orbit, the $130^{\circ}$ scanned sector yields a swath width of $758.5 \mathrm{~km}$ shown in Fig. 1.5. During each scan period, the subsatellite point advances a distance d of $13.9 \mathrm{~km}$. 


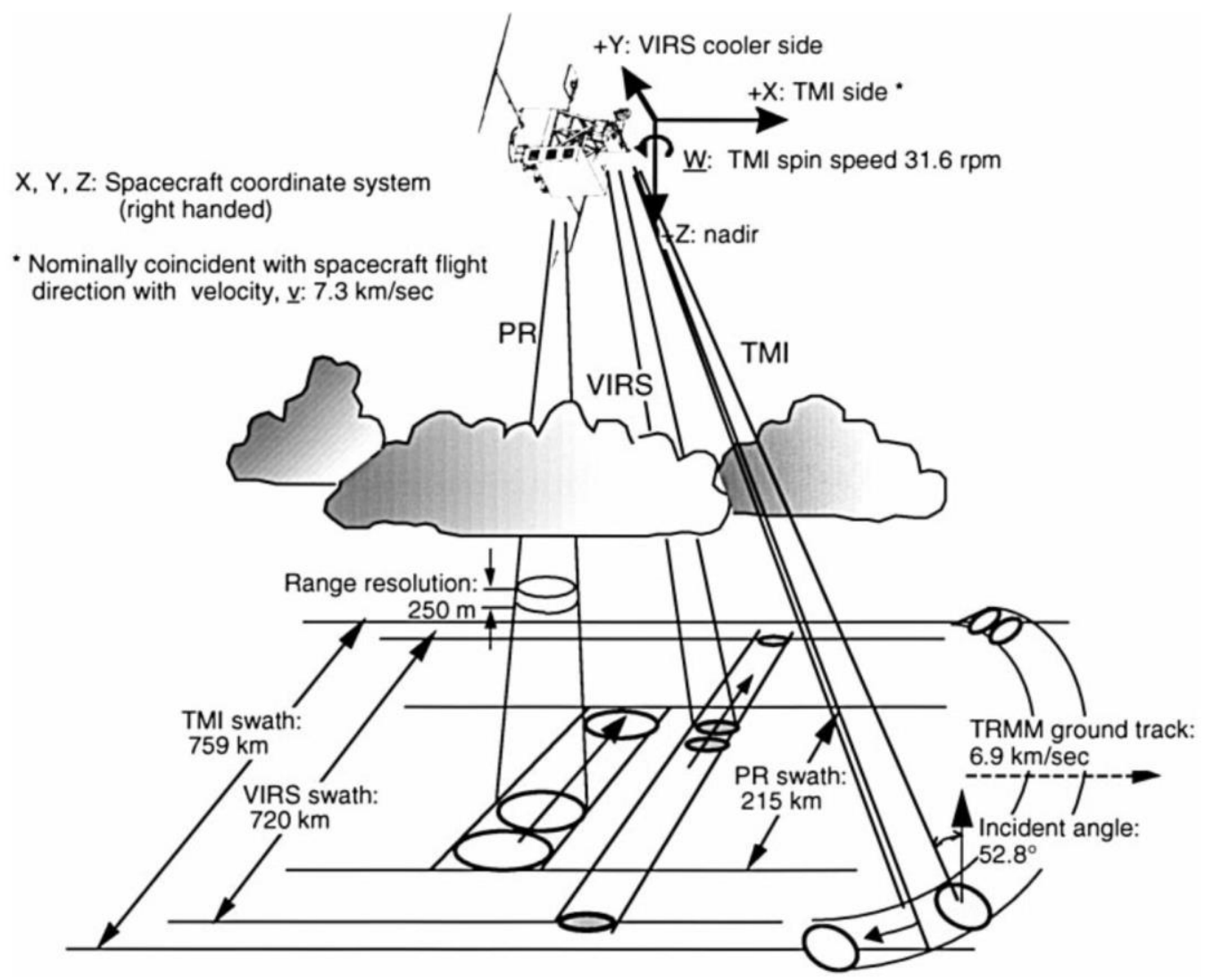

Figure 1.5: Schematic view of the scan geometries of the three TRMM primary rainfall sensors: TRMM Microwave Imager (TMI), Precipitation Radar (PR), and Visible and infrared scanner (VIRS) (Kummerow et al. 1998).

\subsubsection{A12 Retrieval Algorithm}

The TMI surface rainfall estimates of version 7 (V7) (Kummerow et al., 1996, 2001) are used in this study. The TMI 2A12 rainfall algorithm (Kummerow et al. 1996, 2001) retrieves surface rainfall using TMI frequencies $(10,19,21,37$, and $85 \mathrm{GHz})$ using the Goddard Profiling algorithm, which uses a Bayesian approach to represent surface rain. The horizontal resolution of TMI products is $5.1 \times 5.1 \mathrm{~km} 2(4.4 \times 4.4 \mathrm{~km} 2$ before August 2001 orbital boost). 


\section{QUANTIFICATION OF PRECIPITATION ASYMMETRY OF TROPICAL CYCLONES USING 16-YR TRMM OBSERVATIONS}

\subsection{Abstract}

Tropical Cyclone Inner core precipitation and convection asymmetries under the effects of vertical wind shear and storm motion was quantified using the Fourier wavenumber decomposition method upon the pixel level data of 3,542 TRMM TMI overpasses. Composites of wavenumber-1 and wavenumber-1-6 total precipitation asymmetries were constructed to show the distribution pattern under different storm motion speed, vertical wind shear and the combined effects of varying vertical wind shear and storm motion. Results indicate that motion-relative total precipitation asymmetry is generally located down-motion. The phase of motion-relative maximum asymmetry shifts cyclonically by adding the wavenumber-2-6 asymmetry to wavenumber- 1 . Shear is more dominant than motion on the distribution of precipitation asymmetry. Because of the stronger mean shear magnitude in Southern Hemisphere (SH), the asymmetric magnitude of shear-relative composite of $\mathrm{SH}$ is higher than that in Northern Hemisphere (NH). For the CAT 1-2 and CAT 3-5 storms, the light surface rainfall tends to distribute upshear. The moderate and heavy surface rainfall are downshear left. The analysis of combined effects of motion and shear shows when shear is weak, and shear is to the left of motion, the precipitation asymmetry is determined more by storm motion than vertical wind shear. The main contributor to the general asymmetry pattern is from the moderate and heavy precipitation. The maximum asymmetry location resembles the location of the maximum of heavy precipitation. The wavenumber-2-6 energy localizes the maximum heavy 
precipitation asymmetry, and the location changes slightly from the original location of wavenumber-1 maximum asymmetry.

\subsection{Introduction}

Several studies have investigated the influence of storm motion (Miller 1958; Frank 1977; Markes 1985; Burpee and Black 1989; Rodgers et al. 1994; and Lonfat et al. 2004) and vertical wind shear (Corbosiero and Molinari 2002; Cecil 2007; Wingo and Cecil 2010) on the asymmetries present in the rainfall distribution in tropical cyclones (TCs). While studies have most often analyzed these effects separately, only a few studies have addressed the combined effect of storm motion and vertical wind shear on TC precipitation asymmetries (Corbosiero and Molinari 2003; Rogers et al. 2003; Chen et al. 2006; Ueno 2007). Likewise, these studies have typically focused on only the low wavenumber (wavenumber-1) contribution to rainfall asymmetry.

As a consequence of the lack of quantitative measurements of TC rainfall and environmental wind shear over the open ocean, earlier studies focused on the effect of storm motion on TC rainfall asymmetry from either individual case studies (e.g. Miller 1958; Willoughby et al. 1984; Markes 1985; Burpee and Black 1989; Reasor et al. 2000), statistical studies using a small sample size (Frank 1977; Rodgers et al. 1994), or numerical studies (Shapiro 1983; Frank and Ritchie 1999). These studies agree that the rainfall maximum in the inner core region is generally observed in down-motion quadrants, which indicates the importance of frictional convergence in the boundary layer, particularly to the right of motion (Shapiro 1983; Frank and Ritchie 1999). Whereas the approach prior typically focused on case studies, Lonfat et al. (2004, hereafter LMC04) were among the 
first to verify the down motion and right of motion rainfall maxima using a larger sample of storms (260). Their methodology consisted of analyzing motion-relative composites of the Fourier decomposed wavenumber-1 distribution of passive-microwave-derived surface rain rate estimates (2121 instantaneous precipitation observations from 1998 to 2000) from the Tropical Rainfall Measuring Mission (TRMM) microwave imager (TMI).

Similar to the storm-motion relative rainfall asymmetry, studies examining the shear-relative rainfall distribution agree that the wavenumber- 1 asymmetry consists of a downshear rainfall maximum. Many studies arrived at a similar conclusion despite using a variety of datasets. For example, Corbosiero and Molinari (2002) analyzed lightning data from the National Lightning Detection Network (NLDN) to investigate the asymmetry of convection relative to the vertical wind shear at varying radii from the center. They showed that more than $90 \%$ of flashes occurred in the downshear left quadrant within a radius of $300 \mathrm{~km}$. When differentiated by only those within $100 \mathrm{~km}$ the preferred location is slightly downshear left, while between 100 and $300 \mathrm{~km}$ the preference shifted to the downshear right. Cecil (2007) similarly found a downshear left maximum using multi-passive microwave sensors derived rain rates from the Remote Sensing Systems (RSS) retrievals for a large sample of Atlantic storms (1998-2004). Using an improved version of the RSS retrieval, with degraded resolution, Wingo and Cecil (2010) extended Cecil (2007) globally and concluded that the majority of the asymmetry in mean rain rates is accounted for by the heaviest rain rates.

Considering that the storm motion and vertical wind shear both create rainfall asymmetries in TCs, one of the most important questions is which effect is more dominant? Corbosiero and Molinari (2003) investigated the combined effect of shear and motion by 
using the same NLDN dataset as Corbosiero and Molinari (2002). They observed that the front-right quadrant favored by storm motion and the downshear-left quadrant favored by shear often overlap in their sample. By examining subsets where motion and shear favor opposing regions, they found that the environmental shear is the dominant factor modulating the convective activity in hurricanes. In a high-resolution, cloud-resolving numerical model simulation of Hurricane Bonnie (1998), Rogers et al. (2003) also investigated the combined contributions of vertical wind shear and motion to rainfall asymmetry. They found that an along-track shear resulted in a symmetric rainfall accumulation, whereas a cross-track shear created a right-side asymmetry in the accumulated rainfall.

Using the same TRMM dataset and Fourier decomposition method as LMC04, Chen et al. (2006, hereafter CKM06) verified the conclusion of Corbosiero and Molinari (2003) that the vertical wind shear dominates the amplitude of the wavenumber-1 rainfall asymmetries relative to the effect of motion, particularly in a moderate to highly sheared environment (where the asymmetry is predominately downshear left). In a low shear environment, the asymmetry from storm motion had a larger contribution, especially in the outer rainband regions. Similar to CKM06, Ueno (2007) found that the magnitude of asymmetry was greatest when the shear vector and storm motion vector were oriented in the same direction or when the shear vector was right to the motion vector.

Although the wavenumber- 1 asymmetry appears to be dominant, a few studies (CMK06; Reasor et al. 2009; Zhu et al. 2013; Yu et al. 2015) have also suggested that perturbations of higher wavenumbers (greater than one) may also have potentially significant contributions. For example, in a numerical modeling study Zhu et al. (2013) 
showed that the energy from wavenumber 1-6 contributed a large amount of energy to the total perturbation field of potential vorticity, while CKM06 also found that, though the wavenumber-1 is dominant, the wavenumber- 2 rainfall asymmetry still contributes half of the amplitude of wavenumber- 1 asymmetry.

The present study will complement the previous studies by utilizing the Fourier decomposition method on a large sample of satellite-derived rain rates to quantify TC precipitation asymmetries with respect to both storm motion and vertical wind shear. Given the relative lack of studies examining the contributions of higher wavenumbers, in this study, we focus on the rainfall asymmetry component not only from wavenumber-1, but higher wavenumbers (up to 6). In addition to the total precipitation, we will also uniquely analyze the asymmetry of three categorized rain rates: light, moderate, and heavy precipitation. The 16 years of TRMM observations of TC inner cores utilized in the current study is likely, to date, the most robust dataset utilized to examine the TC precipitation distribution. Using this long-term dataset, we will attempt to answer the following questions:

In the energy spectrum:

- How much do higher wavenumbers contribute to the total perturbation energy of TC precipitation compared to the wavenumber-1?

In both a motion-relative and shear-relative framework:

- How much do higher wavenumbers contribute to the amplitude of asymmetry compared to the wavenumber-1?

- Do higher wavenumber asymmetry components change the location of maximum precipitation? 
- How is the maximum asymmetry amplitude and location sensitive to varying storm motion speeds and vertical wind shear magnitudes?

Under the combined effects of shear and motion:

- When does vertical wind shear dominate the TC inner core precipitation distribution over motion, and vice versa?

\subsection{Data and Method}

\subsubsection{Date Sources}

The dataset used in this study is 16-yr (1998-2013) TRMM TMI data from the TRMM Tropical Cyclone Precipitation Feature (TCPF) database (Jiang et al., 2011). The TRMM TCPF database includes TRMM overpasses of all global TCs that reached at least tropical storm intensity during their lifetimes. Tropical Cyclones were separated into six oceanic basins, including Atlantic (ATL), east Pacific (EPA), northwest Pacific (NWP), north Indian Ocean (NIO), south Indian Ocean (SIO), and south Pacific Ocean (SPA). The storm center, intensity, and storm motion speed and direction of each overpass were interpolated from the best-track data. The best-track data of ATL and EPA basins were obtained from the National Hurricane Center hurricane database (HURDAT2). For the rest of basins, the best-track data were from the U.S. Navy's Joint Typhoon Warning Center. On the basis of the maximum sustained wind speed $\left(V_{\max }\right)$ at the TRMM observation time, all TC overpasses were classified into tropical depressions (TDs; $V_{\max }<34 \mathrm{kt}$ ), tropical storms (TSs; $34 \mathrm{kt} \leq V_{\max }<64 \mathrm{kt}$ ), category 1-2 hurricanes (CAT1-2; $64 \mathrm{kt} \leq V_{\max } \leq 95 \mathrm{kt}$ ), and category 3-5 hurricanes (CAT3-5; $V_{\max }>95 \mathrm{kt}$ ) according to the Saffir-Simpson Hurricane Scale. 
The best track interpolated storm centers for TS, CAT1-2, and CAT3-5 overpasses were manually adjusted by Zagrodnik and Jiang (2014) and Tao et al. (2017) in order to better align with the representation of the TRMM Precipitation Radar and/or TMI 37-GHz channel. Sometime the best-track interpolated center could be off the real center by a certain distance ranging from a few kilometers to tens of kilometers. Therefore, the manually re-centered dataset is critical to the success of the Fourier transfer wavenumber decomposition performed in this study. Tropical Depression overpasses are not included in this study due to difficulties of center fixing for these low intensity storms.

Table 2.1: Numbers of selected TMI overpasses for different intensity stages in different basins.

\begin{tabular}{c|cccccc|c}
\hline \hline & ATL & EPA & NWP & NIO & SIO & SPA & TOTAL \\
\hline \multirow{2}{*}{ TS } & 519 & 372 & 511 & 79 & 412 & 160 & \multirow{2}{*}{$2053(58.0)$} \\
& $(62.0)$ & $(65.6)$ & $(45.9)$ & $(79.8)$ & $(62.5)$ & $(59.9)$ & \\
CAT 1-2 & 235 & 122 & 360 & 13 & 161 & 57 & \multirow{2}{*}{$948(26.8)$} \\
& $(28.1)$ & $(21.5)$ & $(32.4)$ & $(13.1)$ & $(24.4)$ & $(21.4)$ & \\
CAT 3-5 & 83 & 73 & 242 & 7 & 86 & 50 & $541(15.2)$ \\
& $(9.9)$ & $(12.9)$ & $(21.7)$ & $(7.1)$ & $(13.1)$ & $(18.7)$ & \\
\hline \multirow{2}{*}{ TOTAL } & 837 & 567 & 1113 & 99 & 659 & 267 & \multirow{2}{*}{3542} \\
\hline \hline
\end{tabular}

The TMI has an $878-\mathrm{km}$ swath $(760 \mathrm{~km}$ before the TRMM orbital boost in August 2001), which is much wider than the TRMM radar's swath width. But still, not all TMI overpasses provided a good coverage for the TC observation. In our study, we select overpasses by using the criterion of capturing the entire inner $200 \mathrm{~km}$ radius from the storm center. We also require that the TC had to remain over water and did not undergo extratropical transition within a future 24-hour period. Finally, 3542 qualified TC overpasses are selected including 2,053 TS, 948 CAT1-2, and 541 CAT3-5 overpasses. Table 2.1 shows the sample sizes for six different basins and 3 different intensity stages. 
The TMI surface rainfall estimates from version 7 (V7) of the TMI 2A12 algorithm (Olson et al., 1996; Kummerow et al., 2001] are used in our study. The 2A12 rainfall algorithm retrieves surface rainfall using TMI frequencies $(10,19,21,37$, and $85 \mathrm{GHz})$ using the Goddard Profiling algorithm (GPROF; Kummerow et al., 2001), which used a Bayesian approach to retrieve surface rain. Using a numerical cloud model, a large dataset of precipitation profiles was generated. Brightness temperatures associated with each simulated profile were calculated using a radiative transfer model. By comparing the observed and model calculated brightness temperatures, the probability that if a simulated profile is likely to be as realistic as an observed profile was calculated. The model profiles were then weighted and summed to composite the best estimate profile of the observation, which was used to determine the rainfall estimate of each observed brightness temperature sets from all frequencies. The algorithm produces rain rates at a horizontal resolution of $5.1 \times 5.1 \mathrm{~km}^{2}\left(4.4 \times 4.4 \mathrm{~km}^{2}\right.$ before August 2001 orbital boost $)$.

In the present study, the TMI $2 \mathrm{~A} 12$ rain rate $(\mathrm{R})$ is categorized into four precipitation types: total precipitation $\left(\mathrm{R} \geq 0.5 \mathrm{~mm} \mathrm{hr}^{-1}\right)$, heavy precipitation $(\mathrm{R} \geq 10 \mathrm{~mm}$ $\left.\mathrm{hr}^{-1}\right)$, moderate precipitation $\left(10 \mathrm{~mm} \mathrm{hr}^{-1}>\mathrm{R} \geq 5 \mathrm{~mm} \mathrm{hr}^{-1}\right)$, and light precipitation (5 mm $\mathrm{hr}^{-1}>\mathrm{R} \geq 0.5 \mathrm{~mm} \mathrm{hr}^{-1}$ ). Since the TMI V7 oceanic algorithm gives a high number of false positive rain rates, we use a threshold rain rate of $0.5 \mathrm{~mm} \mathrm{~h}^{-1}$ with a probability $95 \%$ of rain as the minimum criterion of raining (Zagrodnik and Jiang, 2014).

One additional dataset that supplements the TRMM TCPF database is included to calculate the magnitude and direction of the vertical wind shear. The wind field data are derived from the $0.75^{\circ}$-resolution European Centre for Medium-Range Weather Forecasts (ECMWF) interim reanalysis dataset (Simmons et al. 2007). The vertical wind shear is 
calculated as the difference between the averaged wind vectors at the 200- and 850-hPa. Following Zagrodnik and Jiang (2014) and Tao et al. (2017), the wind vectors are averaged within a ring of 500-750 km from the TC center to eliminate the influence of the storm's circulation as much as possible. As tested by Wingo and Cecil (2010), the rainfall distribution does not appear to be sensitive to the depth of the vertical wind shear calculation, i.e., over the deep layer (200-850 hPa) or shallow layer (500-850, or 700-925 $\mathrm{hPa})$. Therefore, we will only use the deep-layer shear calculation.

Table 2.2: Mean values of current TC intensity $\mathrm{V}_{\max }$, vertical wind shear magnitude, storm motion speed, and numbers (percentages) of selected TMI overpasses with shear same as, opposite to, to the left of, and to the right of motion for the total dataset, different oceanic basins, and different intensity stages.

\begin{tabular}{c|ccccccc}
\hline \hline & Vmax & Shear & Motion & same & opposite & left & right \\
\hline ATL & 61.4 & 10.6 & 5.2 & $104(12.4)$ & $106(12.7)$ & $155(18.5)$ & $472(56.4)$ \\
EPA & 60.6 & 6.1 & 4.6 & $80(14.1)$ & $52(9.2)$ & $246(43.4)$ & $189(33.3)$ \\
NWP & 72.2 & 7.9 & 5.2 & $166(14.9)$ & $95(8.5)$ & $398(35.8)$ & $454(40.8)$ \\
NIO & 51.1 & 10.3 & 3.2 & $26(26.3)$ & $3(3.0)$ & $40(40.4)$ & $30(30.3)$ \\
SIO & 60.9 & 8.9 & 3.9 & $120(18.2)$ & $43(6.5)$ & $358(54.4)$ & $138(20.9)$ \\
SPA & 65.2 & 10.1 & 4.6 & $56(21.0)$ & $22(8.2)$ & $142(53.2)$ & $47(17.6)$ \\
\hline TS & 45.6 & 9.1 & 4.6 & $282(13.7)$ & $206(10.0)$ & $619(30.2)$ & $946(46.1)$ \\
CAT1_2 & 77.5 & 8.6 & 4.9 & $184(19.4)$ & $65(6.9)$ & $244(25.7)$ & $455(48.0)$ \\
CAT3_5 & 114 & 6.9 & 5 & $86(15.9)$ & $50(9.2)$ & $161(29.8)$ & $244(45.1)$ \\
Total & 64.5 & 8.7 & 4.8 & $552(15.6)$ & $321(9.1)$ & $1024(28.9)$ & $1645(46.4)$ \\
\hline \hline
\end{tabular}

In Table 2.2 the mean values of TC intensity, motion speed, and vertical wind shear magnitudes are shown for the six basins and the three intensity groups, respectively. Among the six basins, the average intensity of NWP TC overpasses is the highest. The average intensity of NIO is the lowest. The overall average intensities of the six basins are about $60 \mathrm{kt}$. The motion speeds of all basins are in the range from 3 to $5 \mathrm{~m} / \mathrm{s}$. NIO 
overpasses have the lowest motion speed. The average shear magnitudes of ATL, NIO, and SPA overpasses are about $10 \mathrm{~m} / \mathrm{s}$, which are higher than those of EPA, NWP, and SIO. Among the three intensity groups, it is noteworthy, that TS overpasses have the highest average wind shear magnitude and lowest motion speed. CAT 3-5 overpasses have the lowest average wind shear magnitude and highest motion speed.

\subsubsection{Analysis method}

Similar to LMC04, CKM06, and Yu et al. (2015), the TC rainfall asymmetry is quantified by using Fourier decomposition. Within a polar coordinate using the TC center as the origin, the spatial asymmetries of rainfall are computed by binning TMI rain rates in 10-km-wide annuli from $30-\mathrm{km}$ radius to $200-\mathrm{km}$ radius from the TC center. In each annulus, the Fourier coefficients are computed using the following equations (Stull 1987):

$$
\begin{aligned}
& a_{n}=\frac{1}{N} \sum_{k=0}^{N-1} R(k) \cos (2 \pi n k / N) \\
& b_{n}=-\frac{1}{N} \sum_{k=0}^{N-1} R(k) \sin (2 \pi n k / N)
\end{aligned}
$$

where $R(k)$ is each of the individual rain rate estimates and $n$ is the wavenumber. $N$ is equal to 360 , which is the total number of points being analyzed in each annulus. The variable $\mathrm{N}$ is also the highest wavenumber that we can resolve in this Fourier decomposition. The variable $k$ is the index of each point. The complex formula of Fourier coefficient $c_{n}$ is:

$$
c_{n}=a_{n}+i b_{n}
$$


According to Zhu et al. (2013), the total rainfall perturbation energy through all wavenumbers can be represented as:

$$
\sigma_{\eta}^{2}=\sum_{n=1}^{N-1}|c(n)|^{2}
$$

Then the fractional contribution of each wavenumber $n$ to the total perturbation energy $(E(n))$ is:

$$
E(n)=\frac{\left|c_{n}\right|^{2}}{\sigma_{\eta}^{2}}
$$

We can also call $E(n)$ as the normalized perturbation energy spectrum. Clearly, $E(n)$ provides a way to quantify the contribution of a certain wavenumber to the total perturbation energy as a percentage. By definition, summing $E(n)$ through all wavenumbers will reach unity:

$$
\sum_{n=1}^{N-1} E(n)=1
$$

The rainfall asymmetric component of wavenumber n, $R_{n}$, can be represented by

$$
R_{n}=a_{n} \sin \left(\frac{2 \pi n k}{N}\right)+b_{n} \cos \left(\frac{2 \pi n k}{N}\right)
$$

Note that $R_{n}$ is not divided by the azimuthal mean rain rate (wavenumber $0, R_{0}$ ) as used in LMC04. Instead, we will compare the non-normalized and normalized amplitudes of wavenumber 1 and the sum of wavenumber 1 through wavenumber 6 rainfall components in $\mathrm{mm} \mathrm{hr}^{-1}\left(R_{1}\right.$ and $\left.R_{1-6}\right)$ and percentages $\left(R_{l} / R_{0}\right.$ and $\left.R_{1-6} / R_{0}\right)$ in the composite 2-D distribution Fig. 3. In figures (Fig. 4-7, 9-12, and 14-17) following Fig. 3 the asymmetry components $R_{l}$ or $R_{l-6}$ will be normalized by the azimuthal mean rain rate $\left(R_{0}\right)$ of each annulus in each overpass showing in percentages $\left(R_{1} / R_{0}\right.$ or $\left.R_{1-6} / R_{0}\right)$. 
When the motion-relative rainfall asymmetries are concerned, the rainfall asymmetry is composited relative to the motion vector over each TMI overpass. We stratify the motion speed into two categories: slow motion (motion speed $<5 \mathrm{~m} \mathrm{~s}^{-1}$ ) and fast motion (motion speed $\geq 5 \mathrm{~m} \mathrm{~s}^{-1}$ ). When the shear-relative rainfall asymmetries are concerned, the rainfall asymmetry is composited relative to the shear vector over each TMI overpass. We stratify the shear magnitude into three categories: low shear $\left(0-5 \mathrm{~m} \mathrm{~s}^{-1}\right)$, moderate shear (5$\left.10 \mathrm{~m} \mathrm{~s}^{-1}\right)$, and high shear $\left(>10 \mathrm{~m} \mathrm{~s}^{-1}\right)$. When the combined effect of both motion and shear is concerned, we follow CKM06's methodology to stratify the shear and motion vector angle difference $(d A=$ shear angle - motion angle) into four categories: the shear vectors are in the same $\left(d A \leq 22.5^{\circ}\right)$, opposite $\left(\mathrm{dA} \geq 157.5^{\circ}\right)$, right $\left(22.5^{\circ}<d A<157.5^{\circ}\right)$, or left $\left(-157.5^{\circ}<d A<-22.5^{\circ}\right)$ of the storm motion directions. Table 2.2 shows the number (percentage) of overpasses of the four categories. For each basin, 10 20\% of the total overpasses are categorized as the group of "same." The group of "opposite" has the least number of overpasses, which is $\sim 10 \%$. About $50 \%$ of the overpasses have the shear direction to the right (left in the southern hemisphere) of motion direction and $\sim 20 \%$ of the overpasses have the shear direction to the left (right in the southern hemisphere) of motion direction for ATL, SIO, and SPA. For the basins of EPA and NIO in the northern hemisphere, there are $\sim 40 \%$ overpasses categorized as "left" and $\sim 30 \%$ categorized as "right". For NWP, there are $\sim 35 \%$ in the "left" category and $\sim 40 \%$ in the "right" category. For the three intensity groups, similarly, 10 20\% of the overpasses have the wind shear direction same as the motion direction and $\sim 10 \%$ are categorized as "opposite". When deciding an overpass is categorized as "left" or "right" for the three intensity groups, all the overpasses in the southern hemisphere are mirrored. For all three intensity groups, there 
are $\sim 30 \%$ of the overpasses categorized as "left" and $\sim 45 \%$ categorized as "right". After mirroring the overpasses in southern hemisphere, shear direction to the right of motion direction is the most populated category. When we composite overpasses over all basins (in Figs. 3, 6, 7, 11, 12, 14, 15, 16, and 17), the Southern Hemisphere (SH) overpasses are mirrored relative to either the shear or the motion direction to a Northern Hemisphere $(\mathrm{NH})$ frame of reference to account for the Coriolis effect as in CKM06. For both the motionrelative and shear-relative framework, the phase maximum represents the phase angle of the largest rainfall asymmetry. Also, as pointed by CKM06, "the larger the asymmetry amplitude, the more variability in the spatial distribution of TC rainfall may be explained by the reference system (either the environmental shear relative or the TC motion relative)."

\subsection{Results}

\subsubsection{Rainfall Perturbation Energy Spectrum}

Table 2.3 shows the normalized energy spectrum of rainfall perturbations averaged within $0-200 \mathrm{~km}$ from the storm center and over the annulus from 30 to $80 \mathrm{~km}$ from the storm center. For the entire $0-200 \mathrm{~km}$ region, wavenumber 1 contributes $37 \%$ of the total perturbation energy of total precipitation, but only 19,11 , and $13 \%$ of the total perturbation energy of light, moderate, and heavy precipitation, respectively. It suggests that separating total precipitation into sub-categories breaks perturbations down by producing larger higher wavenumber components. Comparing the contribution of wavenumber 1 with higher wavenumbers, it is found that the energy contribution decreases quickly as the wavenumber gets higher for all 4 precipitation categories. For example, the energy of 
wavenumber 1 is about the double of that of wavenumber 2 , which is about the double of wavenumber 3, so on and so forth. However, the sum of energies from wavenumber 2 through wavenumber 6 is similar to the energy of wavenumber 1 for the total precipitation,

Table 2.3: The percentage of total perturbation energy contributed by wavenumber (WN) $1,2,3,4,5,6,2-6$ (2 through 6 ) and $>6$ perturbations averaged over the annulus from 0 to $200 \mathrm{~km}$ and from 30 to $80 \mathrm{~km}$ from the TC center for total precipitation, light precipitation, moderate precipitation, and heavy precipitation, respectively.

\begin{tabular}{|c|c|c|c|c|c|c|c|c|}
\hline $0-200 \mathrm{~km}$ & WN1 & WN2 & WN3 & WN4 & WN5 & WN6 & WN2-6 & $\mathrm{WN}>6$ \\
\hline $\begin{array}{c}\text { total } \\
\text { precipitation } \\
\text { lioht }\end{array}$ & 37.1 & 14.6 & 7.8 & 4.9 & 3.5 & 2.6 & 33.4 & 29.5 \\
\hline $\begin{array}{l}\text { precipitation } \\
\text { moderate }\end{array}$ & 19.4 & 10.9 & 7.6 & 5.5 & 4.2 & 3.4 & 31.6 & 49.0 \\
\hline $\begin{array}{c}\text { precipitation } \\
\text { heavy }\end{array}$ & 11.3 & 6.7 & 4.9 & 3.9 & 3.3 & 2.8 & 21.6 & 67.1 \\
\hline precipitation & 13.3 & 7.1 & 4.5 & 3.2 & 2.5 & 2.0 & 19.3 & 67.4 \\
\hline $30-80 \mathrm{~km}$ & WN1 & WN2 & WN3 & WN4 & WN5 & WN6 & WN2-6 & $\mathrm{WN}>6$ \\
\hline $\begin{array}{c}\text { total } \\
\text { precipitation } \\
\text { light }\end{array}$ & 46.9 & 15 & 7.1 & 4.1 & 2.7 & 2.0 & 30.9 & 22.2 \\
\hline $\begin{array}{l}\text { precipitation } \\
\text { moderate }\end{array}$ & 22.3 & 13.2 & 8.6 & 5.9 & 4.5 & 3.6 & 35.8 & 41.9 \\
\hline $\begin{array}{c}\text { precipitation } \\
\text { heavy }\end{array}$ & 14.8 & 8.8 & 6.2 & 4.9 & 4.0 & 3.4 & 27.3 & 57.9 \\
\hline precipitation & 19.7 & 8.9 & 5.1 & 3.5 & 2.7 & 2.2 & 22.4 & 57.9 \\
\hline
\end{tabular}

and is about $30 \%$ to $50 \%$ higher than the energy of wavenumber 1 for the light, moderate, and heavy precipitation. Therefore, by analyzing both $R_{l}$ and $R_{1-6}$ in this study, at least $70 \%$ of the total perturbation energy of total precipitation is covered. For the approximate eyewall region (from 30 to $80 \mathrm{~km}$ from the TC center), the wavenumber 1 contributes a 
much higher percentage to the total perturbation energy than for the entire $0-200 \mathrm{~km}$ region for all precipitation categories, especially for the total precipitation. As a result, the energy from wavenumbers higher than 6 is lower than that for the entire $0-200 \mathrm{~km}$ region.

As the energy spectrum can be different at various radii from the TC center, Fig. 2.1 shows the percentage of total perturbation energy contributed by wavenumbers from 1 to 6 , respectively, and the sum of wavenumber 1 through wavenumber 6 (wavenumber 16) as a function of the radial distance. The wavenumber 1 energy is higher in the inner core region (within 0-100 km) than in the outer region for all four precipitation categories. The perturbation energies from wavenumber 2 and higher do not change much with the radial distance. As a result, the sum of energy from wavenumber 1-6 is less in the outer region than in the inner region. For different precipitation types, the stronger the precipitation rate, the less the outer region perturbation energy is contributed by the wavenumber 1-6 energy. 

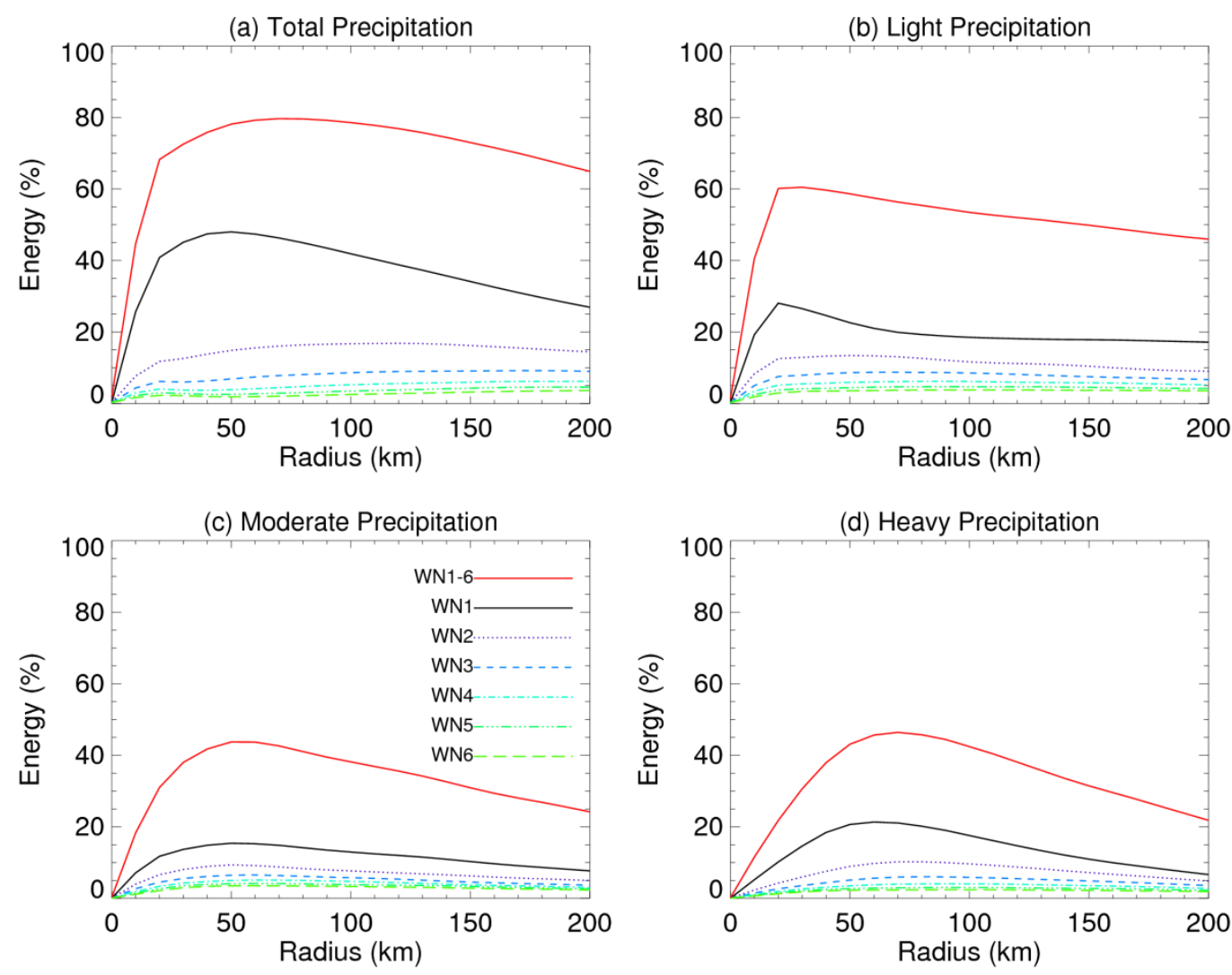

Figure 2.1: The percentage of total perturbation energy contributed by wavenumber (WN) $1,2,3,4,5,6$, and 1-6 (1 through 6) as a function of radial distance for (a) total precipitation, (b) light precipitation, (c) moderate precipitation, and (d) heavy precipitation.

Moderate and heavy precipitation types have more high-wavenumber (higher than wavenumber 6) energy relative to light precipitation. As shown Table 2.3, the perturbation energy from wavenumbers $>6$ contributes about $49 \%$ of the total perturbation energy of light precipitation within $0-200 \mathrm{~km}$ radius, but the contribution from wavenumbers $>6$ increases to $67 \%$ for moderate and heavy precipitation. From both Table 2.2 and Fig. 2.1, we can see that the total precipitation has the highest energy contribution from wavenumber 1-6, followed by the light precipitation. Both moderate and heavy precipitations have lower energy contribution from wavenumber 1-6 than the light precipitation. Stronger convection with moderate or heavy rain rates is usually small in size and distributed randomly 
distributed around the TC center (Tao and Jiang, 2015), which is the characteristic of higher wavenumbers. Tao and Jiang (2015) showed that the shallow precipitation is more symmetrically distributed around the TC center, which is the characteristic of lower wavenumbers.

\subsubsection{Azimuthal mean rain rate}

Tropical Cyclone rainfall asymmetry can be represented by the decomposed field of rain rates either normalized by the azimuthal mean rain rate (LMC04 and CKM06) or not $\mathrm{Yu}$ et al. (2014). Before comparing the two representations of asymmetry, to understand the relative amplitude of the composited field to the mean rain rate, it is important to show how the azimuthal mean rain rates distribute radially among intensity groups, basins, and precipitation groups. For total precipitation, the radial distributions of azimuthally averaged rain rates for each storm intensity groups are shown in Fig. 2.2a. For all three intensity groups (Fig. 2.2a) and six basins (Fig. 2.2b), the unconditional mean rain rate, which takes the azimuthal average counting in the non-raining data point, is higher than 
(a) Intensity Groups

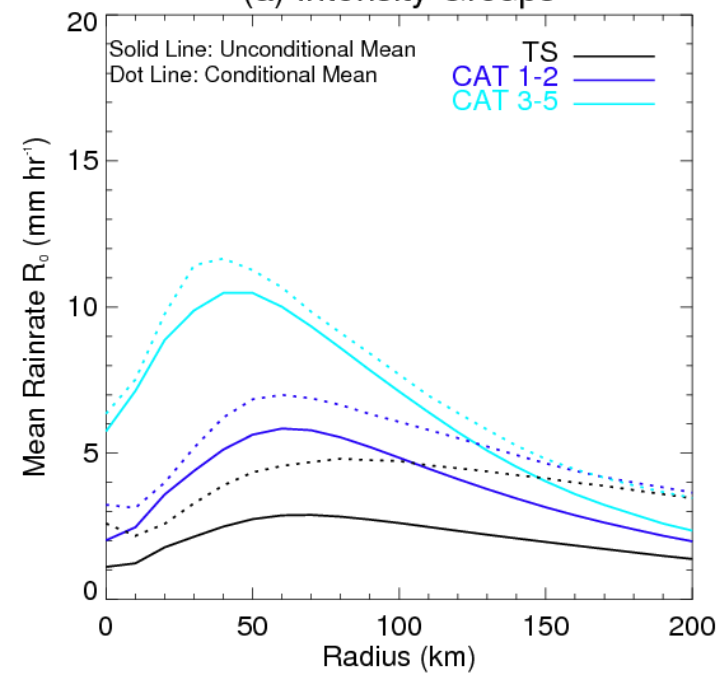

(c) Precipitation Types

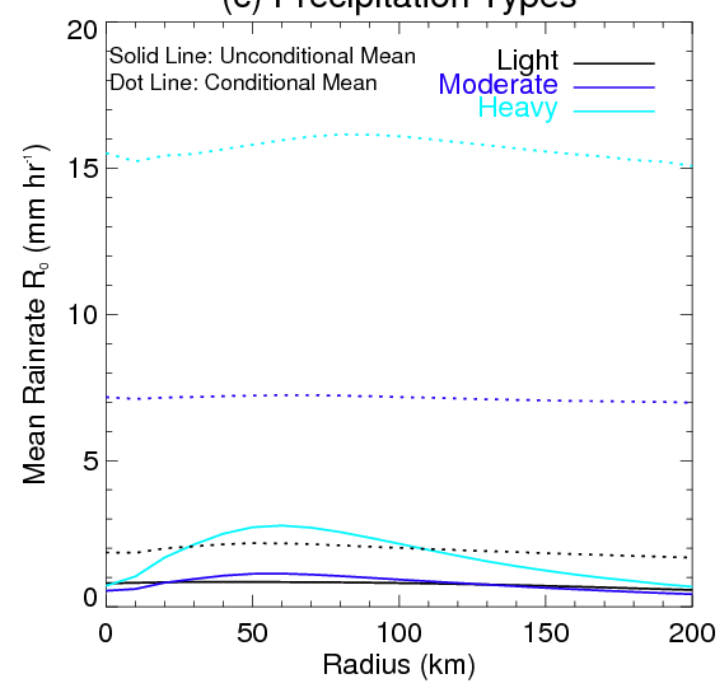

(b) Basins

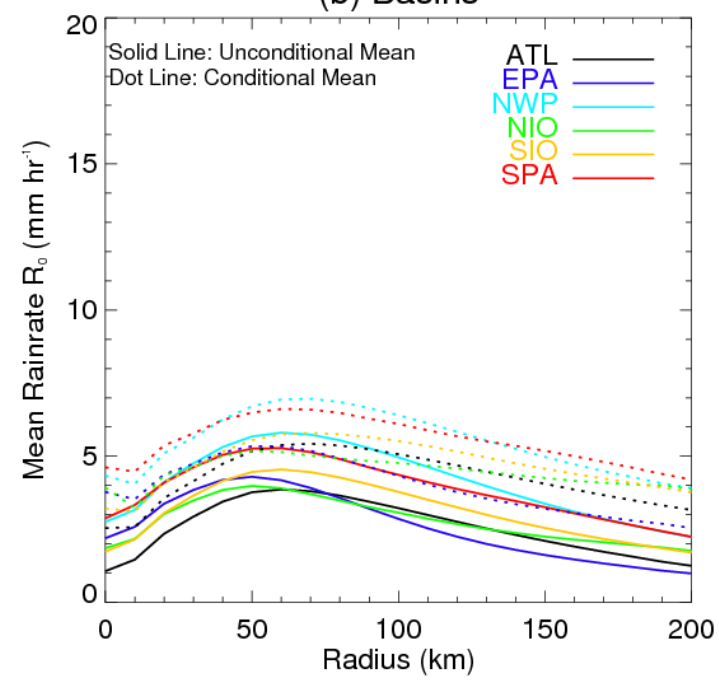

Figure 2.2: Mean rain rates $\left(\mathrm{R}_{0}\right)$ for (a) three different intensity stages (b) six oceanic basins, and (c) three precipitation types.

the conditional mean rain rate (without non-raining data points). However, when normalizing wavenumber 1 or 1-6 perturbation to the mean rain rate, the azimuthal average is the unconditional mean. From the radial distribution of the unconditional mean, it is shown that the peak mean rain rates are 3,6 , and $11 \mathrm{~mm} \mathrm{hr}^{-1}$. The average rain rate increases with radius until it reaches the peak and decreases outward. The location of the peak rain 
rate for TS, CAT 1-2, and CAT 3-5 intensity groups are at about 60, 60, and $40 \mathrm{~km}$ from the storm center, respectively. The radial rain rate distribution is consistent with LMC04 and Marks (1985). Figure 2.2b shows the azimuthal TC mean rain rates in six different basins. By comparing the unconditional mean rain rates, NWP generally has the highest rain rates at all radii. Atlantic tropical cyclone has the lowest mean rain rate in the inner 50 $\mathrm{km}$ region and EPA TC has the lowest mean rain rate in the region from 50 to $200 \mathrm{~km}$ region from the storm center. The mean rain rate variability of each different basins has a similar radial distribution, that the mean rain rate increases from 0 to $50 \mathrm{~km}$ radius and decreases from 50 to $200 \mathrm{~km}$ radius from the storm center. Except for total precipitation, the other three types of precipitation including light, moderate, and heavy precipitation are defined based on certain ranges of rain rate. In Fig. 2.2c, the unconditional and conditional mean rain rate of the three types of precipitation are shown. It shows that the unconditional azimuthal average rain rate reaches its peak around $50-60 \mathrm{~km}$ for all three types of precipitation. The magnitudes of unconditional mean rain rate for light, moderate, and heavy precipitation are smaller than the mean rain rate of their corresponding conditional mean rain rate. The conditional mean rain rates of light, moderate, and heavy precipitation are around 2, 7, and $15 \mathrm{~mm} \mathrm{hr}^{-1}$, respectively. The mean rain rates fall between their corresponding defined ranges for the three types of precipitation.

\subsubsection{Motion effect}

The 2-D motion-relative composites of Fourier decomposed rainfall perturbation and that normalized by azimuthal mean rain rate are shown in Fig. 2.3. The composites (Fig. 2.3Ia,b,c,d) show that the $R_{l}$ maximum is located in down-motion at the radius of 100 
$\mathrm{km}$ for total, moderate, and heavy precipitation. For light precipitation, the $R_{I}$ maximum is located right of motion in the storm inner core with a radius less than $100 \mathrm{~km}$, and located down-motion in the storm outer region. For the wavenumber-1 perturbation normalized by the azimuthal mean rain rates $\left(R_{l} / R_{0}\right)$, the maximum is in down-motion for total, moderate, and heavy precipitation. For light precipitation, the $R_{I} / R_{0}$ maximum is at down-motion right. In LMC04 and CKM06, the rainfall asymmetry is represented by the wavenumber-1 perturbation normalized by the azimuthal mean TMI 2A12 rain rate (corresponding to $R_{I} / R_{0}$ in this paper). The 2 -D rainfall asymmetry is represented by only using the wavenumber-1 perturbation (corresponding to $R_{l}$ in this paper) of 3B42 rain rates in the Fig. 3, 9, and 13 of Yu et al. (2014). Comparing these two ways of representation of rainfall asymmetry, it would be interesting to know the difference between the composite of $R_{1} / R_{0}$ and $R_{1}$ of TMI $2 \mathrm{~A} 12$ data. LMC04's Fig. 17 shows that the maximum wavenumber-1 asymmetry $\left(R_{l} / R_{0}\right.$ in our paper) is in the outer region, which is similar to Fig. 2.3II, while our results in Fig. 2.3I clearly show that the maximum asymmetry is located in the inner core region. LMC04's wavenumber-1 asymmetry was the fraction of the wavenumber-1 asymmetry component normalized by the azimuthal mean. As the radial distance increases from the storm center, the azimuthal mean decreases from $3-13 \mathrm{~mm} / \mathrm{hr}$ in the eyewall region to about $1-3 \mathrm{~mm} / \mathrm{hr}$ in the $200-\mathrm{km}$ radius region (Fig. 2.2a). Therefore, their signal and ours in Fig. 3II and IV were not from the absolute wavenumber-1 asymmetry component alone, but from the wavenumber-1 perturbation relative to the wavenumber 0 . In Fig. 2.3Ia for the total precipitation, the phase maxima are generally down-motion in the front of TC center. The 2-D composites of total (Fig. 2.3Ia) and heavy (Fig. 2.3Id) precipitation both show the maximum $R_{l}$ are located down motion at $100-\mathrm{km}$ radius in 
front of the TC center. The $R_{l}$ composite of light (Fig. 2.3Ib) precipitation has its maximum right of motion from 0-100 $\mathrm{km}$ and down motion from 100-200 $\mathrm{km}$ radius. The phase maximum for $R_{l}$ of moderate precipitation is generally down motion, but the amplitude is small. The maximum perturbation is $0.47 \mathrm{~mm} \mathrm{hr}^{-1}$ for $R_{l}$ of total perturbation, $0.4 \mathrm{~mm} \mathrm{hr}^{-1}$ for $R_{l}$ of heavy precipitation (Table 2.4). However, the maximum perturbations are less than $0.1 \mathrm{~mm} \mathrm{hr}^{-1}$ for $R_{l}$ of both light and moderate precipitation. It implies that most of the precipitation asymmetric perturbations are contributed by the heavy precipitation. The composites of $R_{1-6}$ confirms the dominant contribution from heavy precipitation. As we wonder if this conclusion applies to the composites of $R_{1} / R_{0}$ and $R_{1-6} / R_{0}$, Row II and III shows the asymmetry distribution of the four types of precipitation. In row II, the maximum $R_{I} / R_{0}$ of total precipitation is located down motion. The area of $R_{I} / R_{0}$ higher than $10 \%$ extends from the radius of 50 to $200 \mathrm{~km}$ for total precipitation (Fig. 2.3IIa). Similarly, the area of $R_{l} / R_{0}$ higher than $10 \%$ of heavy precipitation is also located down motion from the radius of 50 to $200 \mathrm{~km}$ (Fig. 2.3IId), though the area is smaller than that of total precipitation. The maximum of $R_{I} / R_{0}$ for light precipitation is located at the $200-\mathrm{km}$ radius (Fig. 2.3IIb) and for moderate precipitation is located down motion from 

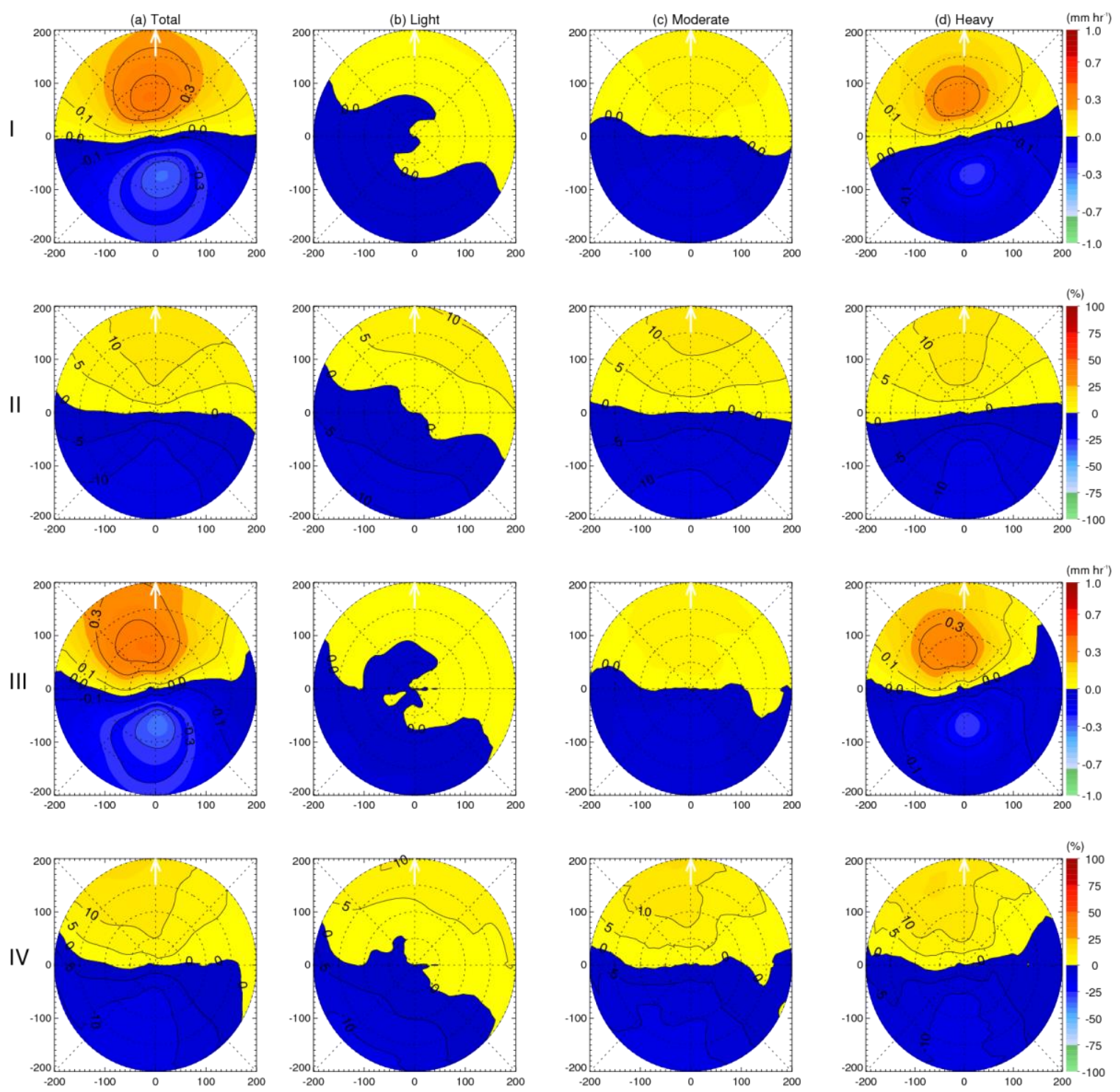

Figure 2.3: The composite motion-relative (Row I) wavenumber 1 asymmetry $\left(\mathrm{R}_{1}\right)$, (Row II) $\mathrm{R}_{1} / \mathrm{R}_{0}$, (Row III) $\mathrm{R}_{1-6}$, (Row IV) $\mathrm{R}_{1-6} / \mathrm{R}_{0}$ of [four columns]: (a) total precipitation, (b) light precipitation, (c) moderate precipitation, and (d) heavy precipitation. The white arrow (pointing upward) represents the orientation of motion vector. The color scale represents average perturbation of the sum of the wavenumber 1 or 1-6 asymmetry components. Dotted range rings represent the 50-, 100-, 150-, and 200-km radii.

100 to $200 \mathrm{~km}$ (Fig. $2.3 \mathrm{IIc}$ ). The composite of $R_{l} / R_{0}$ and $R_{1-6} / R_{0}$ also confirms the prominent contribution from heavy precipitation. It can be noticed for both $R_{1-6} / R_{0}$ and $R_{1-6}$ the location 
of phase maximum changes slightly but generally aligns with the composite of $R_{l} / R_{0}$ and $R_{1}$.

Table 2.4: (for Fig. 3) The asymmetry index (AI) of the composite motion-relative (Row I) wavenumber 1 asymmetry ( $\mathrm{R}_{1}$ ), (Row II) $\mathrm{R}_{1} / \mathrm{R}_{0}$, (Row III) $\mathrm{R}_{1-6}$, (Row IV) $\mathrm{R}_{1-6} / \mathrm{R}_{0}$ of [four columns]: (a) total precipitation, (b) light precipitation, (c) moderate precipitation, and (d) heavy precipitation. The percentage in each pair of parentheses represents the percentage increase of the asymmetry index from $R_{1}$ to $R_{1-6}$ or from $R_{1} / R_{0}$ to $R_{1-6} / R_{0}$.

\begin{tabular}{l|cccc}
\hline \hline AI & (a) Total & (b) Light & (c) Moderate & (d) Heavy \\
\hline$R_{1}\left(\mathrm{~mm} \mathrm{hr}^{-1}\right)$ & 0.47 & 0.05 & 0.09 & 0.4 \\
$R_{1} / R_{0}(\%)$ & 12.9 & 10.6 & 12.5 & 12 \\
$R_{1-6}\left(\mathrm{~mm} \mathrm{hr}^{-1}\right)$ & $0.48(2 \%)$ & $0.04(-20 \%)$ & $0.11(22 \%)$ & $0.41(2 \%)$ \\
$R_{1-6} / R_{0}(\%)$ & $16.4(27 \%)$ & $10.1(-5 \%)$ & $16.4(31 \%)$ & $17(42 \%)$ \\
\hline
\end{tabular}

Apart from the phase maximum changes, it is also interesting to notice the changes in the asymmetry amplitude when adding wavenumber $2-6$. In Table 2.4 , the maxima of $R_{1}, R_{1} / R_{0}, R_{1-6}$, and $R_{1-6} / R_{0}$ corresponding to Fig. 2.3 are shown in I, II, III, and III rows, respectively. For each TC rainfall field, the most noticeable character is the maximum rain rate. The maximum of the composite $R_{1}$ and $R_{1-6}$ shows small perturbation magnitude (Table 2.4). For total and heavy precipitation, the perturbation amplitudes are $\sim 1 \mathrm{~mm} \mathrm{hr}^{-1}$. For light and moderate the perturbation amplitudes are $\sim 0.1 \mathrm{~mm} \mathrm{hr}^{-1}$. There are two possible explanations for the small perturbation amplitudes. Firstly, it could be explained by the motion reference system. The organization of rainfall may not be regularly located at a particular position relative to TC moving direction. When compositing the rain rate perturbation, the maximum perturbations could not often be added up. Instead, the positive maximum perturbations could be diminished by the negative perturbations. Secondly, it 
could also be a result of that the perturbation $\left(R_{l}\right)$ is not normalized by the azimuthal mean $\left(R_{1}\right)$. When compositing, TCs with light rain rate will lower down the composited maximum values. By adding wavenumber 2-6 perturbation to wavenumber 1 , the maximum $R_{l}$ of total, light, moderate, and heavy precipitation increases $2 \%,-20 \%, 22 \%$, $2 \%$, respectively, to $R_{1-6}$. Similarly, the values of the maximum $R_{1-6}$ are as small as $R_{1}$. And the increases (in the time domain) are not consistent with the energy increase in frequency domain by wavenumber 2-6 as mentioned in 2.4.1.

When the perturbation normalized by the azimuthal mean rain rate, the maximum $R_{I} / R_{0}$ of total, light, moderate, and heavy precipitation increase $27 \%,-5 \%, 31 \%$, and $42 \%$ to $R_{1-6} / R_{0}$. After normalization, the increase is more reasonable and consistent with the energy increase in the frequency domain. The maximum $R_{l} / R_{0}$ increase of light precipitation is not consistent with the energy increase. As Tao and Jiang (2015) showed that the shallow precipitation is more symmetrically distributed around the TC center, light precipitation is not necessarily perpetually located at one particular position relative to storm motion. So, when compositing the perturbation of light precipitation, the maximum perturbation of each TC does not necessarily add up. It illustrates that to normalize the perturbation by the azimuthal mean rain rate will better represent the weighted rain rate values of each individual TC and signify the maximum perturbation and location. As quantifying the relative impact of motion on the rainfall asymmetry is a necessary next step to the results in LMC04 and CKM06, in this paper, a wavenumber-based asymmetry index 
(a) ATL Total Precipitation WN 1
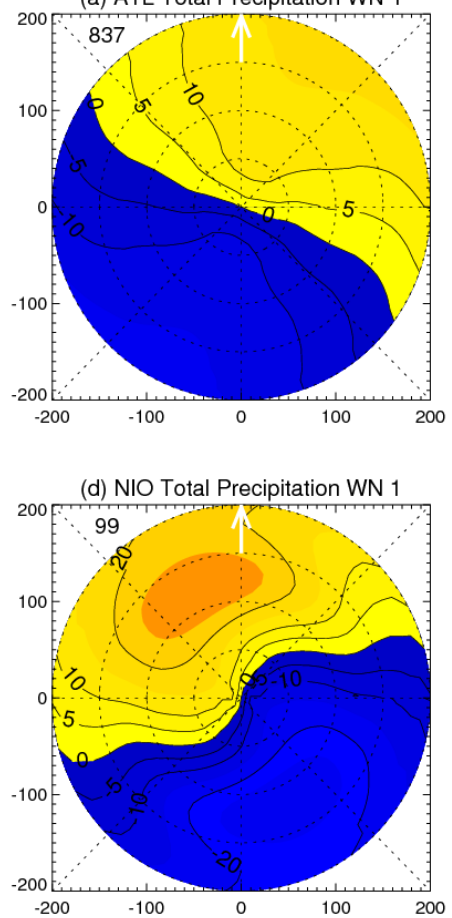

(b) EPA Total Precipitation WN 1
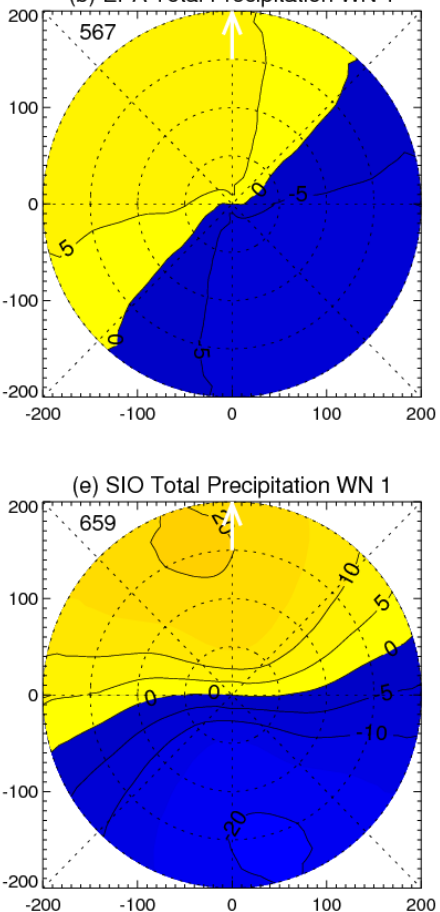

(c) NWP Total Precipitation WN 1
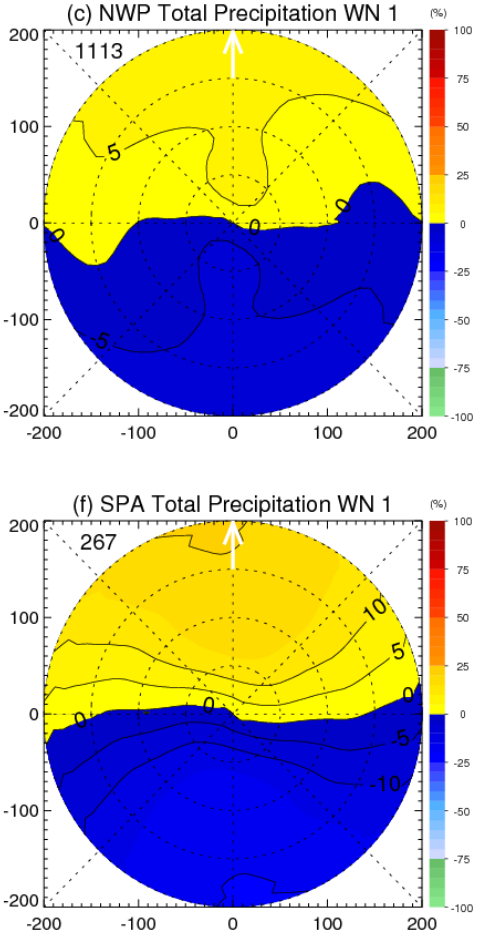

Figure 2.4: The composite motion-relative wavenumber 1 asymmetry normalized by the azimuthal mean $\left(\mathrm{R}_{1} / \mathrm{R}_{0}\right)$ of total precipitation for six basins. The white arrow (pointing upward) represents the orientation of motion vector. The color scale represents average perturbation of the sum of the wavenumber 1 or 1-6 asymmetry components. Dotted range rings represent the 50-, 100-, 150-, and 200-km radii.

is defined as the maximum of the $R_{l} / R_{0}$ or $R_{1-6} / R_{0}$ composite. Alvey et al. (2015) and Tao et al. (2017) defined an asymmetry index through a summation of four relevant absolute quadrant differences of polarized corrected brightness temperature or rainfall frequency in shear-relative coordinate. However, the wavenumber-based asymmetry index in the current paper is based on the Fourier decomposed perturbations of rain rate. Since the decomposed perturbations explicitly represent the precipitation asymmetry, there is no need to calculate the degree of rainfall asymmetry through quadrant differences. A large asymmetry index value signifies a high degree of asymmetry. 
Figure 2.4 presents the composite motion-relative wavenumber-1 asymmetry normalized by the azimuthal mean $\left(R_{1} / R_{0}\right)$ of total precipitation over six oceanic basins. The location of maximum $R_{l} / R_{0}$ of total precipitation is clearly down-motion, especially in NWP and SPA (Figs. 2.4c, e, f). It is consistent with earlier observational studies showing that the rainfall maximum in the inner core region is mainly located at down-motion quadrants (Miller 1958; Willoughby et al. 1984; Markes 1985; Burpee and Black 1989; Reasor et al. 2000) and theoretical modeling studies showing that the boundary layer convergence and upward motion are in down-motion quadrants as well (Shapiro 1983; Frank and Ritchie 1999). In ATL (Fig. 2.4a), there is a right of motion component; while in EPA (Fig. 2.4b), SIO (Fig. 2.4e) and NIO (Fig. 2.4d), there is a left of motion component. The results in the ATL, EPA, NWP, NIO, and SIO are generally consistent with the results shown in LMC04's Fig. 18 for the inner core region (within 100-km radius). The ATL result of down-motion right preference is also similar to Corbosiero and Molinari (2003), which showed a down-motion right maximum of lightning distributions in ATL hurricanes. However, our result in SPA basin is different from that of LMC04. In the SPA, LMC04 showed a down-motion left preference, which is different with the down-motion preference as shown here in Figs. 2.4f. It is noted that LMC04's sample includes quite a lot overpasses in which the TC was not fully captured by the TMI. In LMC04, the criterion of overpass selection was that at least $20 \%$ of the total area within $500-\mathrm{km}$ radius is covered by the TMI. In contrast, we require $100 \%$ coverage by the TMI for the area within $200-\mathrm{km}$ radius. The sample selection difference may be the main reason for the difference between our results and LMC04's. 
(a) ATL Total Precipitation WN 1-6
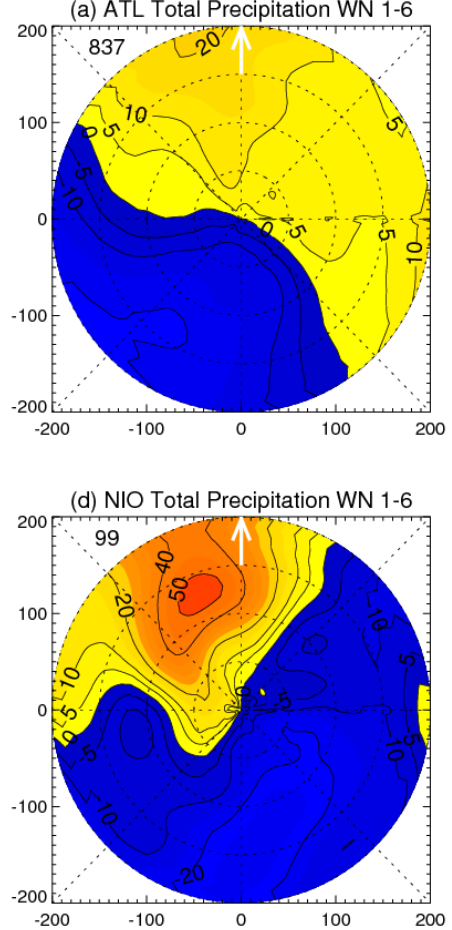

(b) EPA Total Precipitation WN 1-6
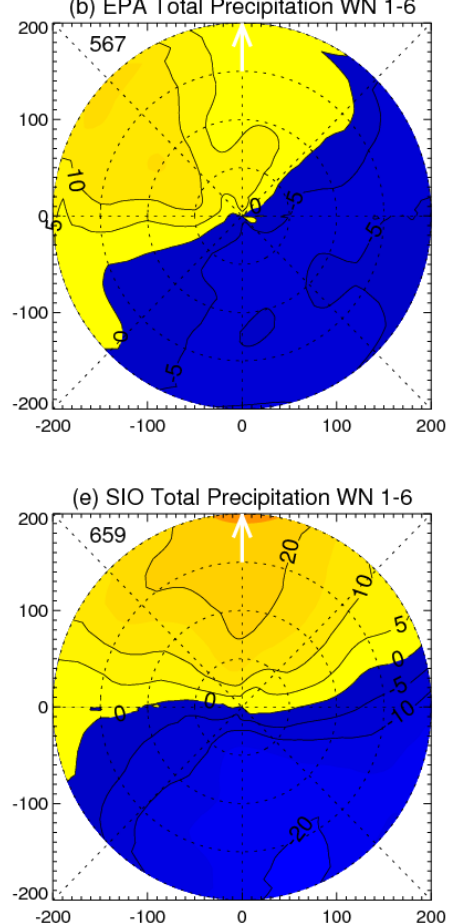
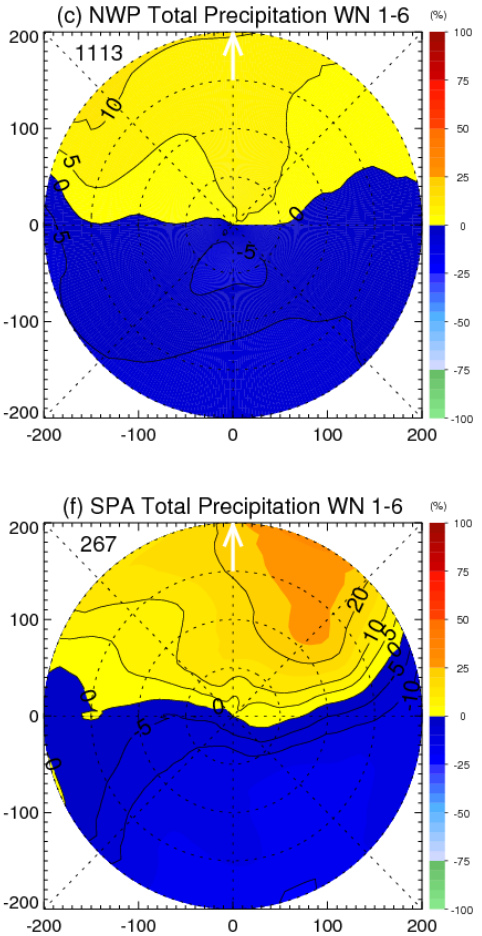

Figure 2.5: Same as FIG. 2.4, but for $\mathrm{R}_{1-6} / \mathrm{R}_{0}$.

Fig. 2.5 presents the composite motion-relative wavenumber 1-6 asymmetry normalized by the azimuthal mean $\left(R_{1-6} / R_{0}\right)$ of total precipitation over six oceanic basins. Compared with the normalized wavenumber 1 asymmetry shown in Fig. 2.4, the amplitude of the motion-relative wavenumber 1-6 asymmetry is higher. However, it is interesting to note that, by adding wavenumbers 2-6 to wavenumber 1 , the location of the maximum wavenumber 1-6 asymmetry shifts cyclonically around the TC center in all the basins.

For all the basins, the asymmetry index of $R_{l} / R_{0}$ in Table 2.5 ranks from the highest to the lowest as NIO, SIO, SPA, ATL, EPA, and NWP. That of $R_{1-6} / R_{0}$ ranks from the highest to the lowest as NIO, SPA, SIO, ATL, EPA, and NWP. NIO has both highest $R_{l} / R_{0}$ and $R_{1-6} / R_{0}$ asymmetry index, which means the precipitation field in NIO TCs are distributed at a similar position relative to storm motion direction. Adding wavenumber 26 to wavenumber 1 perturbation, the increase of the asymmetry index is highest for NIO 
(81\%) and is lowest for ATL (21\%). It indicates that NIO has more energy contributed to the positive perturbation than other basins from wavenumber 2-6 and ATL has the least energy from wavenumber 2-6 contributed to the positive perturbation.

Table 2.5: (for Fig. 2.4 and 2.5) The asymmetry index of the composite motion-relative wavenumber 1 and wavenumber 1-6 asymmetry normalized by the azimuthal mean $\left(R_{1} / R_{0}\right.$ and $R_{1-6} / R_{0}$ ) of total precipitation for six basins. The percentage in each pair of parentheses represents the percentage increase of asymmetry index from $R_{1} / R_{0}$ to $R_{1-6} / R_{0}$.

\begin{tabular}{l|cccccc}
\hline \hline $\mathrm{AI}$ & ATL & EPA & NWP & NIO & SIO & SPA \\
\hline$R_{l} / R_{0}(\%)$ & 17.6 & 10.2 & 9.2 & 30.3 & 21 & 20.6 \\
& 21.3 & 18 & 13 & 54.7 & 26.9 & 32.2 \\
$R_{l-6} / R_{0}(\%)$ & $(21 \%)$ & $(76 \%)$ & $(41 \%)$ & $(81 \%)$ & $(28 \%)$ & $(56 \%)$ \\
\hline
\end{tabular}

The motion-relative composite of $R_{l} / R_{0}$ is shown in Fig. 2.6I for the three intensity groups of TS, CAT 1-2, and CAT 3-5. The composites of both the TS and CAT 1-2 show that $R_{1} / R_{0}$ maximum is located down-motion. The $R_{l} / R_{0}$ maximum of CAT 3-5 is located down-motion right. LMC04 also shows the down-motion rainfall asymmetry that is correlated with the asymmetry in the tangential wind circulation. However, there are three noticeable differences in this paper from $\mathrm{LMC} 04$. The first one is that the $R_{l} / R_{0}$ maximum of CAT 3-5 reaches its maximum at the distance from 10 to $125 \mathrm{~km}$ to storm center. The second difference is that the motion relative composite $R_{l} / R_{0}$ of CAT 3-5 has up-motion positive perturbations (Fig. 2.6 column (c)). The third shown in Table 2.6 is that the 
(a) TS Total Precip. All Motion
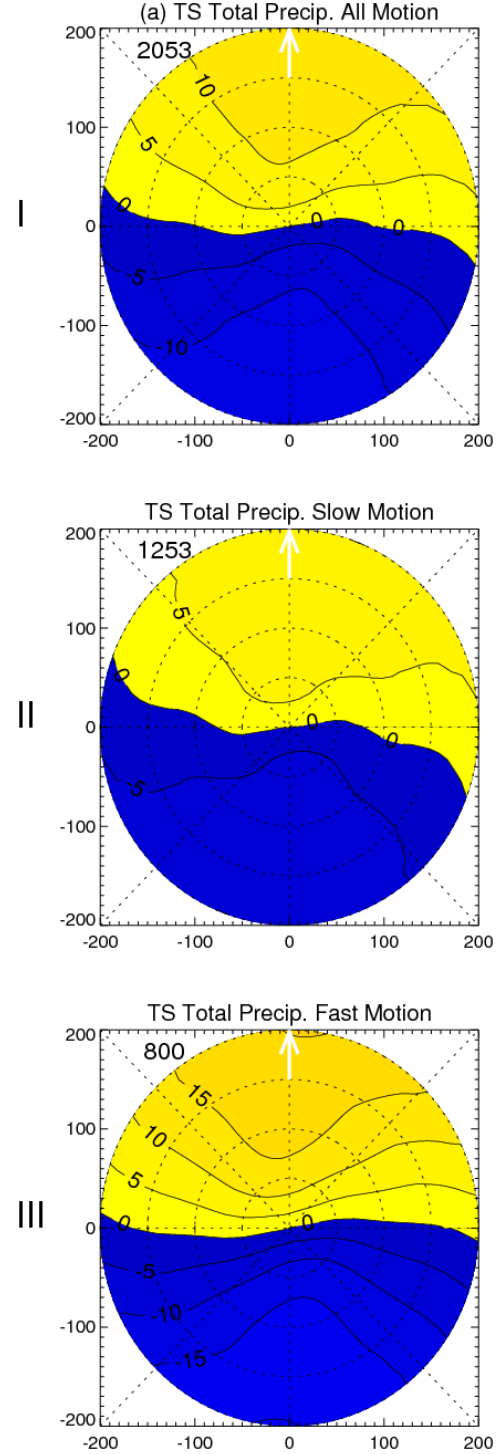
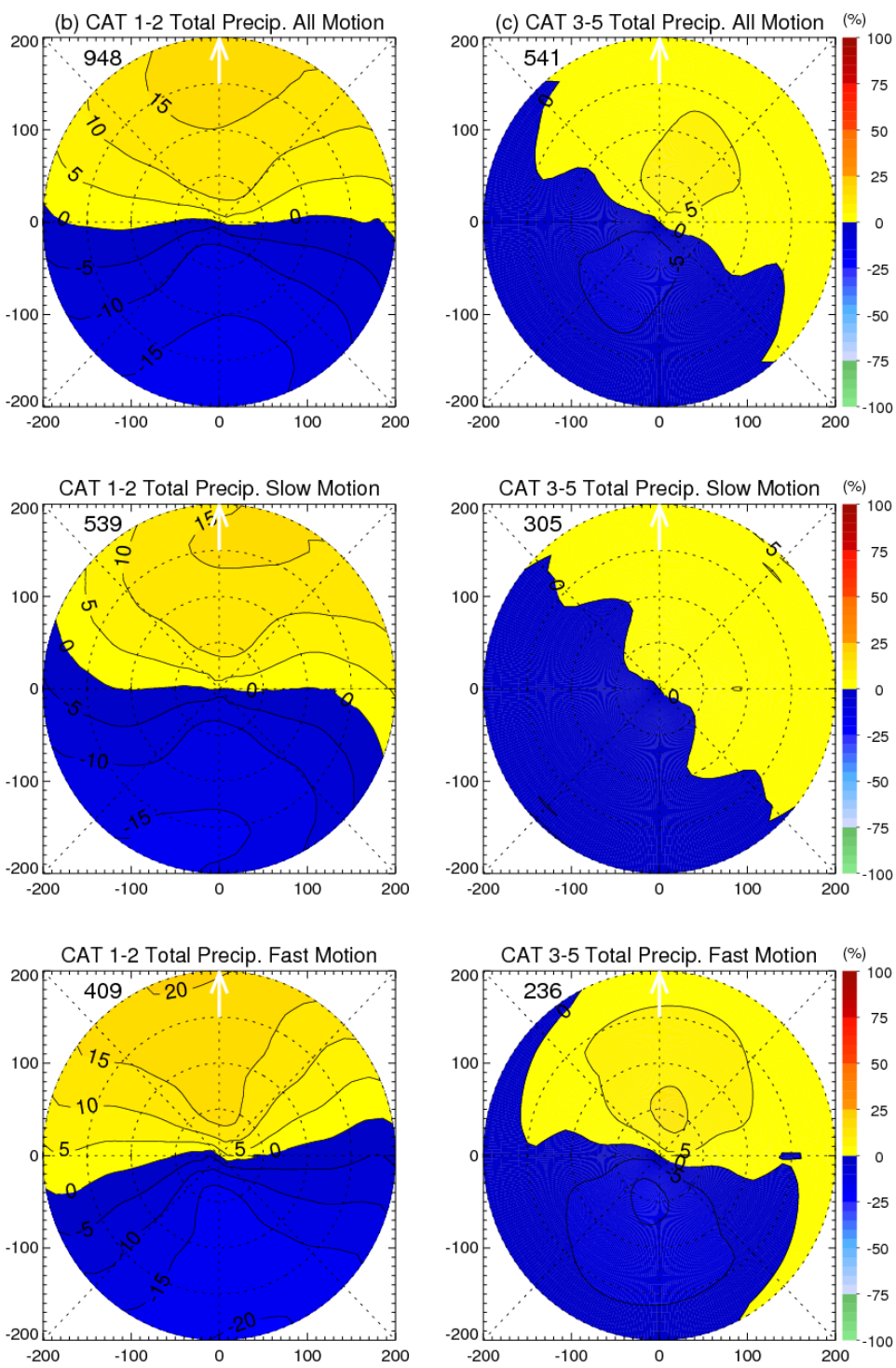

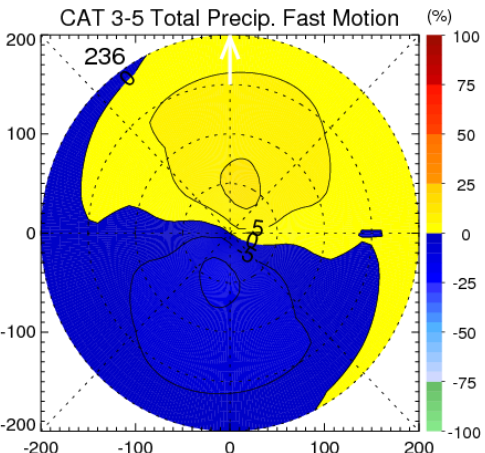

Figure 2.6: The composite motion-relative $\mathrm{R}_{1} / \mathrm{R}_{0}$ of total precipitation for (Row I) all TCs, (Row II) slow moving TCs, and (Row III) fast moving TCs of [three columns] (a) TS, (b) CAT1-2, (c) CAT3-5. The white arrow (pointing upward) represents the orientation of motion vector. The color scale represents average perturbation of the sum of the wavenumber 1 or 1-6 asymmetry components. Dotted range rings represent the 50-, 100-, $150-$, and 200-km radii.

asymmetry index of $R_{l} / R_{0}$ is highest for CAT $1-2(17.5 \%)$, which is higher than that of TS (13.8\%) and CAT 3-5 (8.9\%). Though as the storm intensity increases, the primary circulation should be stronger and more symmetric (e.g., LMC04; Marks and Houze 1987), Fig. 2.6I and Table 2.6 show that the composite of $R_{l} / R_{0}$ of CAT $1-2$ is the more asymmetric 
than TS. However, the composite of $R_{l} / R_{0}$ of CAT 3-5 is still the most symmetric. It indicates that for storm intensity under Category 3, faster-moving speed is not necessarily leading more asymmetric precipitation. As Klotz and Jiang (2017) the reason that the composite of CAT 1-2 is more asymmetric than that of TS can be explained that the lower asymmetry of TS relative to motion is more impacted by motion than vertical wind shear. The collective importance of the TC motion and intensity on rainfall asymmetries is illustrated by dividing the dataset into slow $\left(<5 \mathrm{~m} \mathrm{~s}^{-1}\right)$ and fast $\left(>5 \mathrm{~m} \mathrm{~s}^{-1}\right)$ moving TCs for the three intensity groups (Fig. 2.6). Table 2.6 corresponds to Fig. 2.6 shows that for TS the asymmetry index of $R_{1} / R_{0}$ composite of fast motion is $20.1 \%$, which is greater than that of all motion (13.8\%). Slow motion TS has the smallest asymmetry index of $R_{l} / R_{0}$ composite. As the motion speed increases, TS storms shows more asymmetric $R_{l} / R_{0}$ perturbation. For CAT 1-2 and 3-5 storms, it also shows more asymmetric precipitation perturbation field as the motion speed increases.

The phases of maximum $R_{l} / R_{0}$ of TS are down motion for all, slow-moving, and fast-moving TS (Fig. 2.6 (a) column). For CAT 1-2 (Fig. 2.6 (b) column), the $R_{l} / R_{0}$ maximum for all is located down motion. For slow-moving CAT $1-2$, the $R_{l} / R_{0}$ maximum is located down-motion right. For fast-moving CAT $1-2$, the $R_{l} / R_{0}$ maximum is located down-motion left. For CAT 3-5 (Fig. 2.6 (c) column), the $R_{l} / R_{0}$ maximum for all is located down-motion right. For slow-moving CAT 3-5, the $R_{1} / R_{0}$ maximum is located downmotion right to right of motion. For fast-moving CAT 3-5, the $R_{l} / R_{0}$ maximum is located down motion right. The reason that slow-moving CAT 3-5 has right-of-motion component compared with the fast-moving CAT 3-5 could be because motion has fewer effects than tangential circulation. 
The motion-relative composite of $R_{1-6} / R_{0}$ is shown in Fig. 2.7I for the three intensity groups of TS, CAT 1-2, and CAT 3-5. The composite of TS shows that $R_{1-6} / R_{0}$ maximum is located down-motion left. Compared with the $R_{1} / R_{0}$ composite of TS in Fig. 2.6I, the maximum shift from down motion to down-motion left. Similarly, the maximum $R_{1-6} / R_{0}$ composite of CAT 1-2 in Fig. 2.7Ib is also located down-motion left and the maximum $R_{I} / R_{0}$ composite of CAT 1-2 in Fig. 2.6Ib is located down motion. For CAT 3-5, the $R_{I-}$ ${ }_{6} / R_{0}$ composite has its largest area higher than $5 \%$ down motion right, which is similar to Fig. 2.6Ic. But after adding the perturbation of wavenumber 2-6 to wavenumber 1, in Fig. 2.7Ic, it shows that there are two positive $R_{1-6} / R_{0}$ components higher than $5 \%$. One of them is located at up motion right, and the other is located at down motion left. Perturbations of wavenumber 2-6 contributes more details. The asymmetry indexes of the composite motion-relative $R_{1-6} / R_{0}$ composites and their increases from $R_{1} / R_{0}$ are shown for TS, CAT 1-2, CAT 3-5 with all motion speed in Row I of both sub-tables of Table 2.6. After adding the perturbation of wavenumber 2-6, the asymmetry index of CAT 1-2 composite increases the most, which is higher than that of TS (increased 28\%). The asymmetry index of CAT 3-5 increases the least amount. 
(a) TS Total Precip. All Motion
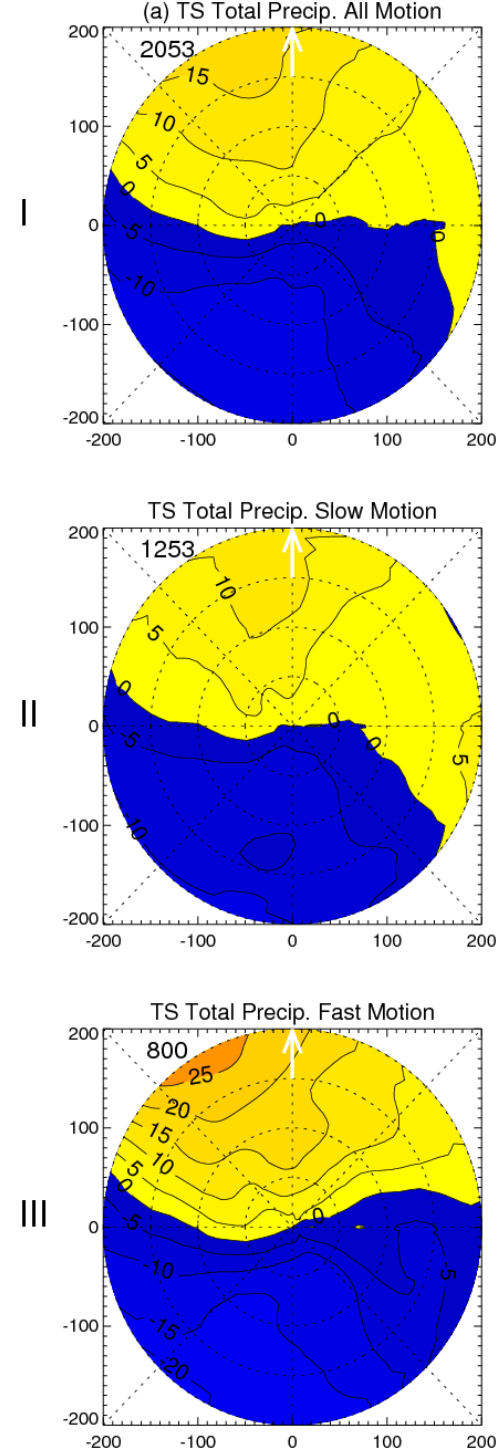

(b) CAT 1-2 Total Precip. All Motion
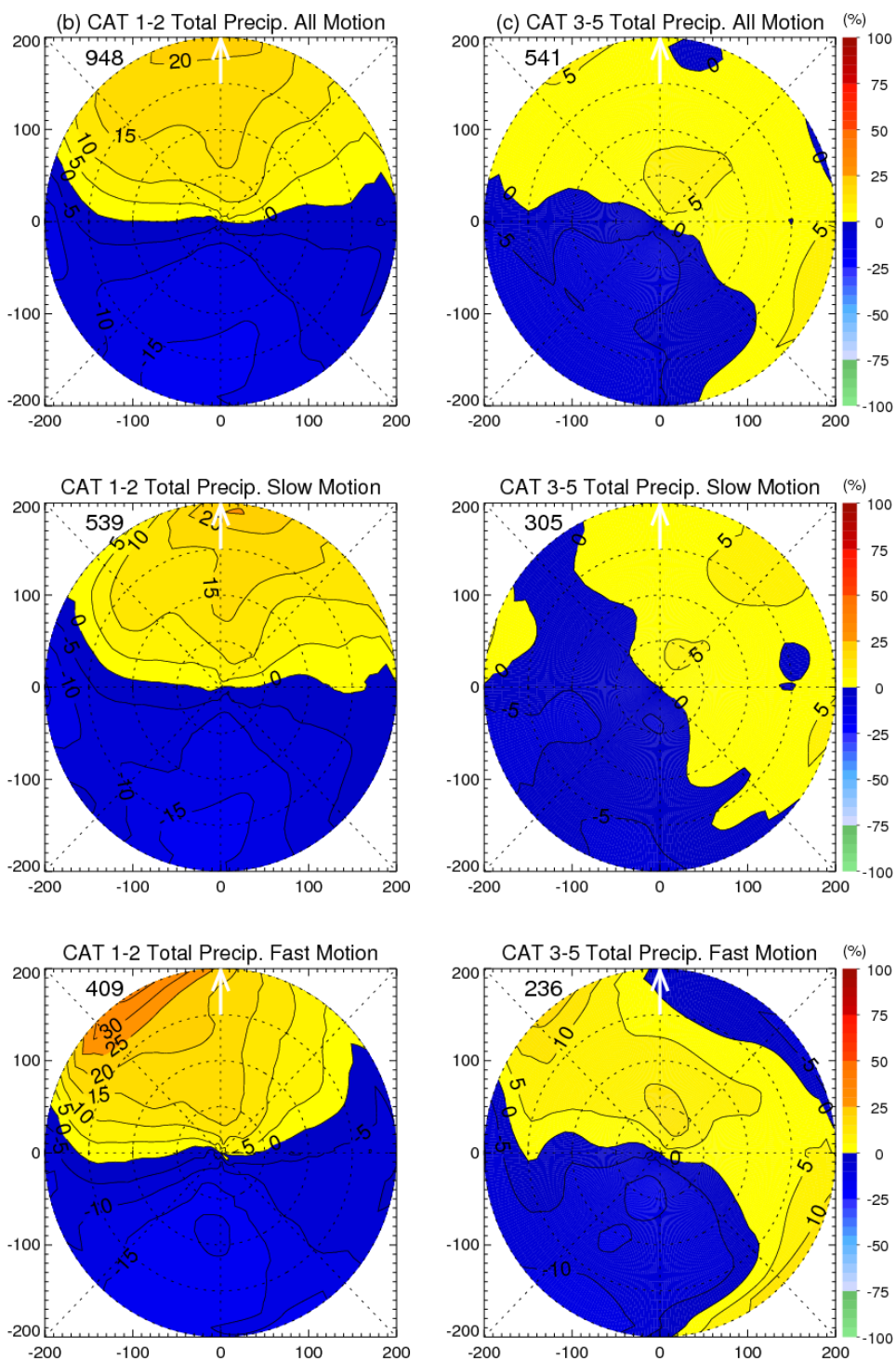

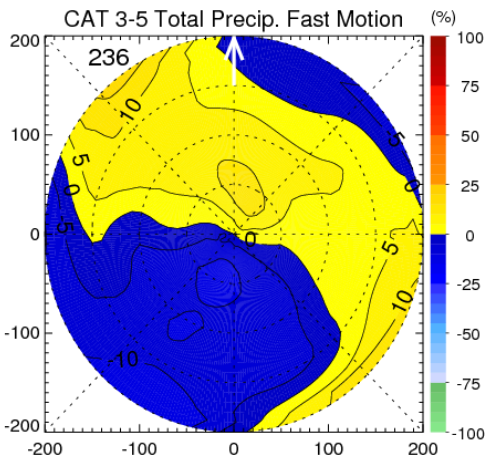

Figure 2.7: Same as FIG. 2.6, but for $\mathrm{R}_{1-6} / \mathrm{R}_{0}$.

The motion-relative composite of $R_{1-6} / R_{0}$ is shown in Fig. $2.7 \mathrm{II}$ for the three intensity groups with slow motion speed. The composite of TS with slow motion speed shows that $R_{1-6} / R_{0}$ maximum is located down-motion left. Compared with the $R_{l} / R_{0}$ composite of TS with slow motion speed in Fig. 2.6II, the maximum shift from down motion to down-motion left. Comparing Fig. 2.7 II with the $R_{l} / R_{0}$ composite of CAT 1-2 
with slow motion speed in Fig. 2.6II, the maximum of $R_{I-6} / R_{0}$ composite stays down motion as that of $R_{1} / R_{0}$. For CAT 3-5, the $R_{1-6} / R_{0}$ composite has its largest maximum area down motion right, which the largest maximum area higher than 5\% is larger than that area higher than 5\% shown in Fig. 2.6IIc. After adding the perturbation of wavenumber 2-6 to wavenumber 1, in Fig. 2.7IIc, it also shows that there are two more positive $R_{1-6} / R_{0}$ components higher than 5\%. One of them is located at down-motion right near the TC center, and the other is located at up-motion right at the radius of $200 \mathrm{~km}$. The asymmetry indexes of the composite motion-relative $R_{1-6} / R_{0}$ composites and their increases from $R_{1} / R_{0}$ are shown for TS, CAT 1-2, CAT 3-5 with slow motion speed in Row II of both sub-tables of Table 2.6. After adding the perturbation of wavenumber 2-6, the asymmetry index of slow-moving CAT 3-5 composite increases the most, which is higher than that of CAT 12 (increased 52\%). The asymmetry index of slow-moving TS increases the least amount $(35 \%)$.

The motion-relative composite of $R_{1-6} / R_{0}$ is shown in Fig. 2.7III for the three intensity groups with fast motion speed. The composite of TS with fast motion speed shows that $R_{I-6} / R_{0}$ maximum is located down-motion left. Compared with the $R_{I} / R_{0}$ composite of TS with fast motion speed in Fig. 2.6III, the maximum shift from down motion to downmotion left. Comparing Fig. 2.7 III with the $R_{I} / R_{0}$ composite of CAT 1-2 with fast motion speed in Fig. 2.6III, the maximum of $R_{1-6} / R_{0}$ composite also shift from down motion to down-motion left. For CAT 3-5, the $R_{1-6} / R_{0}$ composite has its largest maximum area down motion left at $200-\mathrm{km}$ radius. By adding wavenumbers $2-6$ to wavenumber 1, the location of the maximum $R_{1-6} / R_{0}$ shifts cyclonically around the TC center for all three intensity groups. There is also another area higher than 5\% located down-motion right in Fig. 7IIIc, 
which corresponds to the largest maximum area in Fig. 2.6IIIc. It indicates that the high wavenumber changes more of the outer region $(\sim 200 \mathrm{~km})$. The asymmetry indexes of the composite motion-relative $R_{1-6} / R_{0}$ composites and their increases from $R_{1} / R_{0}$ are shown for TS, CAT 1-2, CAT 3-5 with fast motion speed in Row III of both sub-tables of Table 2.6. After adding the perturbation of wavenumber 2-6, the asymmetry index of fast-moving CAT 1-2 composite increases the most, which is higher than that of TS (increased 40\%). The asymmetry index of fast-moving CAT 3-5 increases the least amount (33\%).

Table 2.6: (for Fig. 6 and 7) The asymmetry index of the composite motion-relative $R_{1} / R_{0}$ and $\mathrm{R}_{1-6} / \mathrm{R}_{0}$ of total precipitation for (Row I) all TCs, (Row II) slow moving TCs, and (Row III) fast moving TCs of [three columns] (a) TS, (b) CAT1-2, (c) CAT3-5. basins. The percentage in each pair of parentheses represents the percentage increase of asymmetry index from $\mathrm{R}_{1} / \mathrm{R}_{0}$ to $\mathrm{R}_{1-6} / \mathrm{R}_{0}$.

\begin{tabular}{l|ccc}
\hline \hline Max. $R_{1} / R_{0}(\%)$ & TS & CAT 1-2 & CAT 3-5 \\
\hline All Motion & 13.8 & 17.5 & 8.9 \\
Slow Motion & 10 & 16.6 & 5.8 \\
Heavy Motion & 20.1 & 20.6 & 12.9 \\
\hline & & & \\
\hline Max. $R_{1-6} / R_{0}(\%)$ & TS & CAT 1-2 & CAT 3-5 \\
\hline All Motion & $17.6(28 \%)$ & $23.5(34 \%)$ & $11.2(26 \%)$ \\
Slow Motion & $13.5(35 \%)$ & $25.3(52 \%)$ & $9.7(67 \%)$ \\
Heavy Motion & $28.2(40 \%)$ & $35.9(74 \%)$ & $17.1(33 \%)$ \\
\hline
\end{tabular}


(a) Category groups

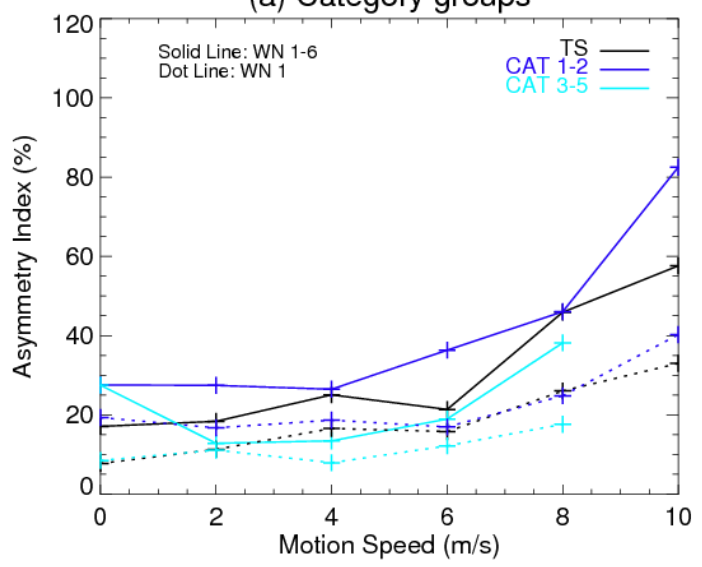

(b) Basins

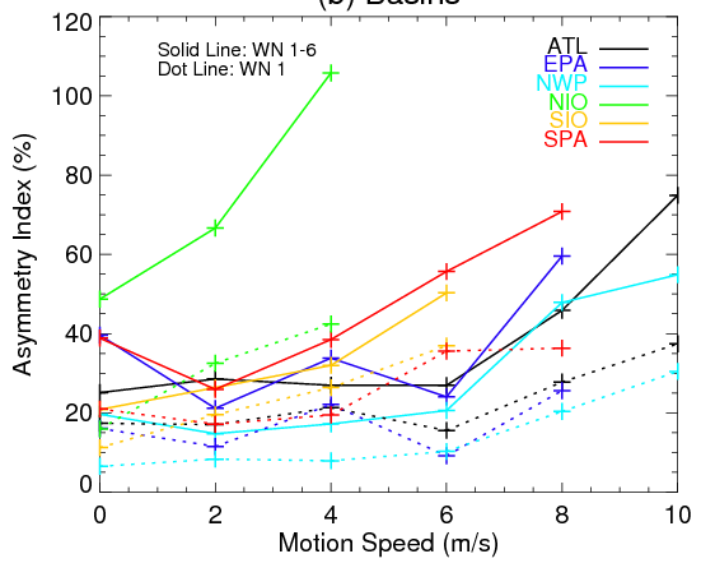

Figure 2.8: The motion-relative wavenumber-based Asymmetry Index (integrated from 0 to $200 \mathrm{~km}$ radius) of total precipitation as a function of motion speed for (a) three different intensity stages with solid line for WN 1-6 and dot line for WN 1, and (b) six different basins with solid line for WN1-6 and dot line for WN 1.

Using the 2-D composites of $R_{1-6} / R_{0}\left(R_{l} / R_{0}\right)$ of each TC overpasses binning in different motion speeds, the motion-relative wavenumber-based asymmetry index is shown in Fig. 2.8 for wavenumber 1 and 1-6. Figure 2.8 shows wavenumber 1 and wavenumber 1-6 asymmetry indices as a function of TC moving speed for the three intensity categories and six basins. Fig. 2.8a shows that as the motion speed increases, the asymmetry index of wavenumber 1-6 also increase accordingly. Among the three intensity categories, CAT 12 has the largest value of asymmetry index of wavenumber $1-6$. From 0 to $10 \mathrm{~m} / \mathrm{s} \mathrm{motion}$ speed, the asymmetry index of wavenumber 1-6 of CAT $1-2$ increases from $\sim 30 \%$ to $\sim 80 \%$. The asymmetry index of wavenumber $1-6$ of TS increases from $\sim 20 \%$ to $\sim 50 \%$. CAT $3-5$ has the smallest value of asymmetry index ranging from $10 \%$ to $40 \%$, which has a slight decrease from 0 to $2 \mathrm{~m} / \mathrm{s}$ and increase from 2 to $8 \mathrm{~m} / \mathrm{s}$. It is consistent with the 2-D composite that $R_{1-6} / R_{0}\left(R_{1} / R_{0}\right)$ of CAT $1-2$ is more impacted by motion. It also shows that the moving speed of most CAT 3-5 TC does not exceed $8 \mathrm{~m} / \mathrm{s}$. Among all the basins, the 
asymmetry index of NIO shows the largest. As TC motion speed increases from 0 to $4 \mathrm{~m} / \mathrm{s}$, the asymmetry index of $R_{1-6} / R_{0}$ for NIO increase from $50 \%$ to $110 \%$. The $R_{1-6} / R_{0}$ asymmetry indexes of SIO and SPA for motion speed from 0 to $8 \mathrm{~m} / \mathrm{s}$ are smaller than NIO but higher than the other basins. The magnitudes of $R_{1-6} / R_{0}$ asymmetry indexes of ATL and EPA are at a similar range for each motion speed bin. NWP has the smallest $R_{1-6} / R_{0}$ asymmetry index. The asymmetry index of wavenumber 1-6 is generally $1 \sim 1.5$ times larger than wavenumber 1. All differences in asymmetry described here are statistically significant at $95 \%$.

\subsubsection{Shear effect}

Figure 2.9 presents the composite shear-relative $R_{l} / R_{0}$ of total precipitation over six oceanic basins. The location of maximum $R_{l} / R_{0}$ is clearly downshear-left in the northern hemisphere $(\mathrm{NH})$ and downshear-right in the southern hemisphere $(\mathrm{SH})$. It is consistent with many previous studies (e.g., Corbosiero and Monlinari, 2002; CMK06; Cecil, 2007; Ueno, 2007; Wingo and Cecil, 2010). Comparing Table 2.5 and 2.7, the $R_{l} / R_{0}$ asymmetry index of each basin relative to vertical wind shear is almost twice as large as that relative to storm motion. In agreement with CKM06, our results strongly suggest that the vertical wind shear is a more important factor in producing a precipitation asymmetry. The asymmetry indexes of the composite $R_{l} / R_{0}$ relative to vertical wind shear of ATL, NIO, SIO, and SPA are higher than $40 \%$, which are higher than EPA and NWP. The $R_{l} / R_{0}$ composite of each basin (ATL, NIO, SIO, and SPA) with asymmetry index greater than 
$40 \%$ corresponds to higher vertical wind shear (Table 2.2) than EPA and NWP, for which the asymmetry index are less than $40 \%$.
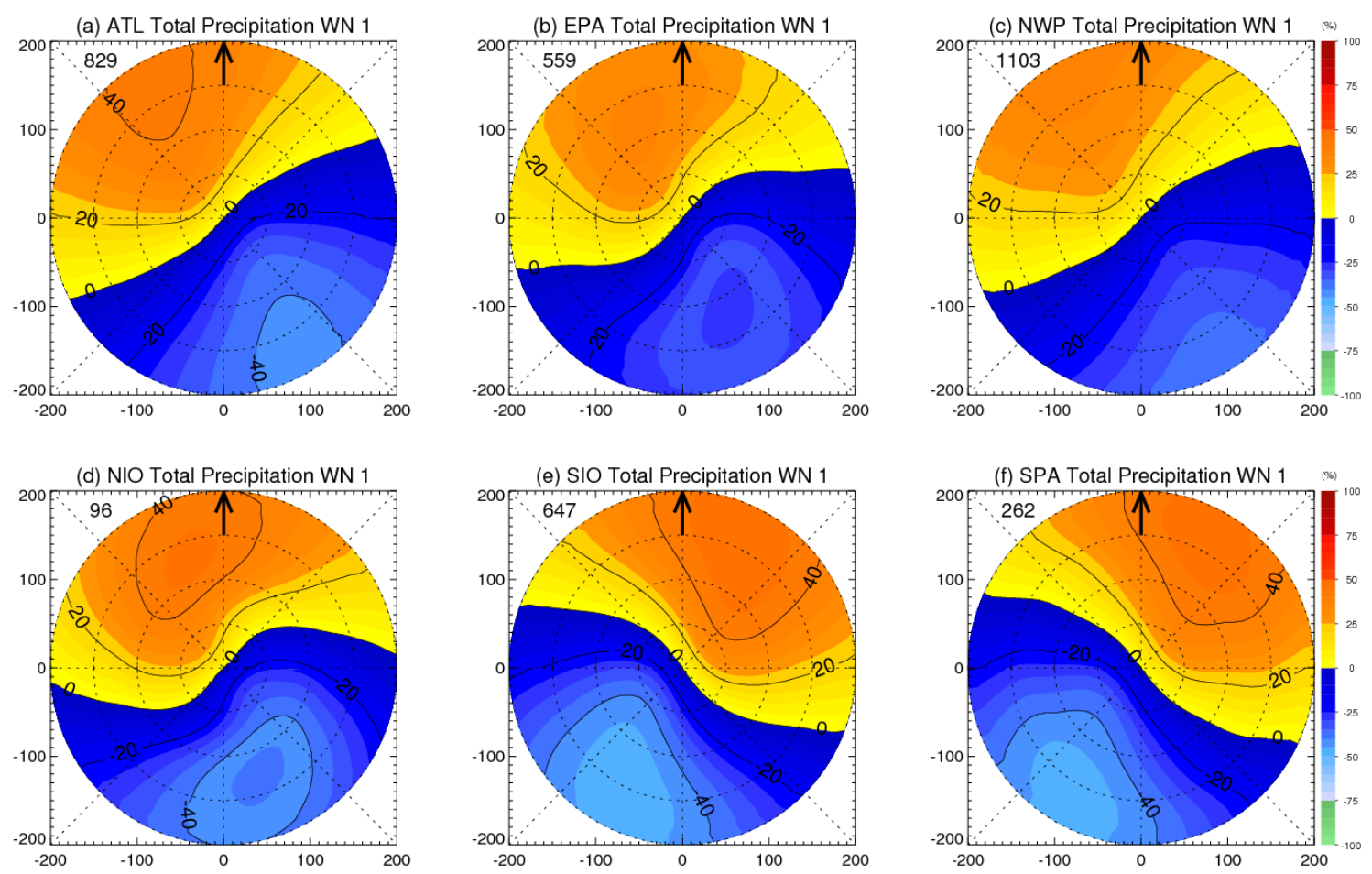

Figure 2.9: The composite shear-relative wavenumber 1 asymmetry normalized by the azimuthal mean $\left(\mathrm{R}_{1} / \mathrm{R}_{0}\right)$ of total precipitation for six basins. The black arrow (pointing upward) represents the orientation of motion vector. The color scale represents average perturbation of the sum of the wavenumber 1 asymmetry components. Dotted range rings represent the 50-, 100-, 150-, and 200-km radii.

The composite shear-relative $R_{1-6} / R_{0}$ of total precipitation over six oceanic basins is shown in Fig. 2.10. Compared Fig. 2.9, the locations of maximum in Fig. 2.10 are also at downshear-left for northern hemisphere and downshear-right for the southern hemisphere. The asymmetry index of the shear-relative $R_{1-6} / R_{0}$ is about $50 \%$ to $70 \%$ higher than $R_{1-6} / R_{0}$ for each basin as shown in Table 2.7. SPA has the largest increase (70\%) from $R_{1} / R_{0}$ to $R_{1-6} / R_{0}$ by adding wavenumber 2-6 to wavenumber 1 . South Indian Ocean has the smallest increase (44\%). For ATL, EPA, NWP, and NIO, the increase is $~ 50 \%$. By adding wavenumbers 2-6 to wavenumber 1 , there is not a cyclonical location shift of the maximum 
wavenumber 1-6 asymmetry around the TC center as in the motion-relative composite.

However, the location of maximum $R_{1-6} / R_{0}$ is more localized than $R_{l} / R_{0}$ which has a broader distribution of the positive perturbation.

(a) ATL Total Precipitation WN 1-6
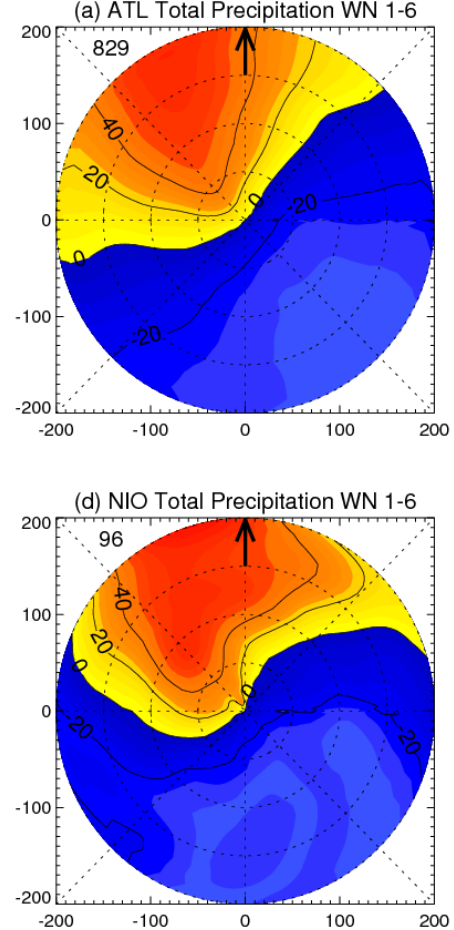

(b) EPA Total Precipitation WN 1-6
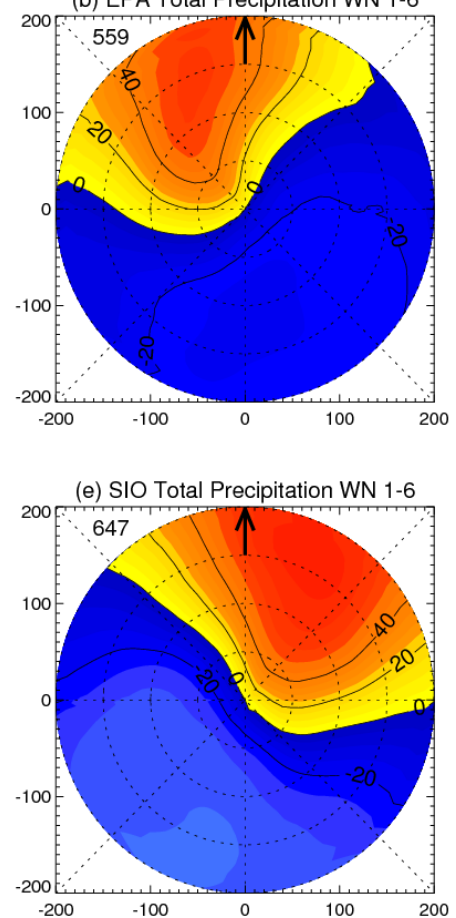

(c) NWP Total Precipitation WN 1-6
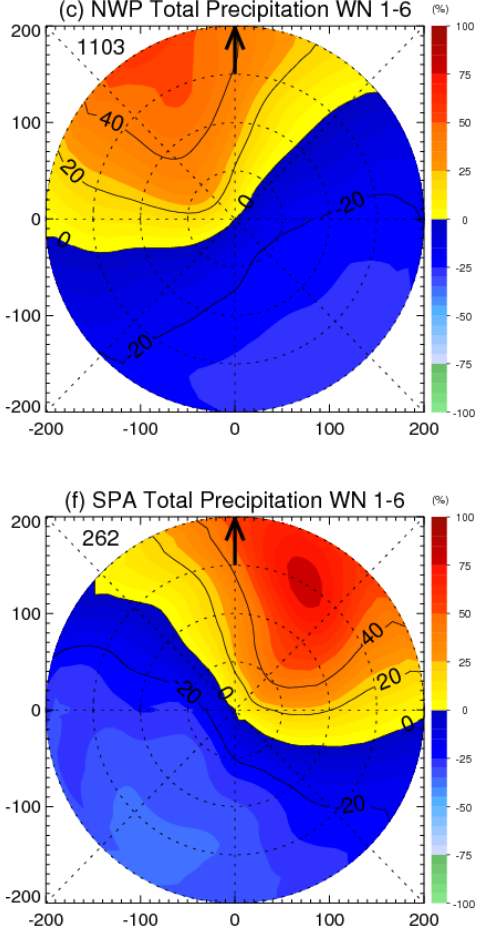

Figure 2.10: Same as FIG. 2.9, but for $\mathrm{R}_{1-6} / \mathrm{R}_{0}$. 
Table 2.7: (for Fig. 9 and 10) The asymmetry index of the composite shear-relative wavenumber 1 and wavenumber 1-6 asymmetry normalized by the azimuthal mean $\left(R_{1} / R_{0}\right.$ and $\mathrm{R}_{1-6} / \mathrm{R}_{0}$ ) of total precipitation for six basins. The percentage in each pair of parentheses represents the percentage increase of asymmetry index from $R_{1} / R_{0}$ to $R_{1-6} / R_{0}$.

\begin{tabular}{l|cccccc}
\hline \hline $\mathrm{AI}$ & ATL & EPA & NWP & NIO & SIO & SPA \\
\hline$R_{I} / R_{0}(\%)$ & 42.2 & 36.7 & 37.9 & 47.2 & 49 & 48.2 \\
& 65.4 & 58.4 & 57.4 & 75.7 & 70.5 & 81.7 \\
$R_{I-6} / R_{0}(\%)$ & $(55 \%)$ & $(59 \%)$ & $(51 \%)$ & $(60 \%)$ & $(44 \%)$ & $(70 \%)$ \\
\hline
\end{tabular}

The effect of shear strength on $R_{l} / R_{0}$ distribution are demonstrated by dividing the TC dataset into three subsets with shear values $<5$ (low shear), 5-10 (moderate shear), and $>10$ (high shear) $\mathrm{m} \mathrm{s}^{-1}$ for the three intensity groups (Fig. 2.11). For TCs in moderate and high shear, the maximum asymmetry is in downshear-left. However, when the shear magnitude is less than $5 \mathrm{~m} \mathrm{~s}^{-1}$, the asymmetry of CAT 3-5 is distributed more towards downshear at the radius of 150 to $200 \mathrm{~km}$. For TS and CAT $1-2$, the $R_{l} / R_{0}$ maximum is mainly downshear left. However, in CKM06, when the shear is less than $5 \mathrm{~m} / \mathrm{s}$, the asymmetry does not have a dominant downshear-left pattern, instead a downshear pattern. In Table 2.8, the asymmetry indexes corresponding to the groups in Fig. 2.11 are shown. For low shear, moderate, and high shear groups, the asymmetry indexes are $\sim 20 \%, \sim 40 \%$, and $60 \%$, respectively. Every $5 \mathrm{~m} / \mathrm{s}$ increment of shear magnitude, there is a $\sim 20 \%$ increment of asymmetry index for each intensity group. The asymmetry indexes of $R_{l} / R_{0}$ for TS with low shear and moderate shear are smaller than those of CAT 1-2 with low and moderate shear. The asymmetry indexes of $R_{1} / R_{0}$ for CAT $1-2$ with low, high, and moderate shear magnitude are all higher than those of CAT 3-5. As the storm intensity getting higher, the precipitation is more symmetric with the exception of CAT 1-2 with 
(a) TS Total Precip. Low Shear
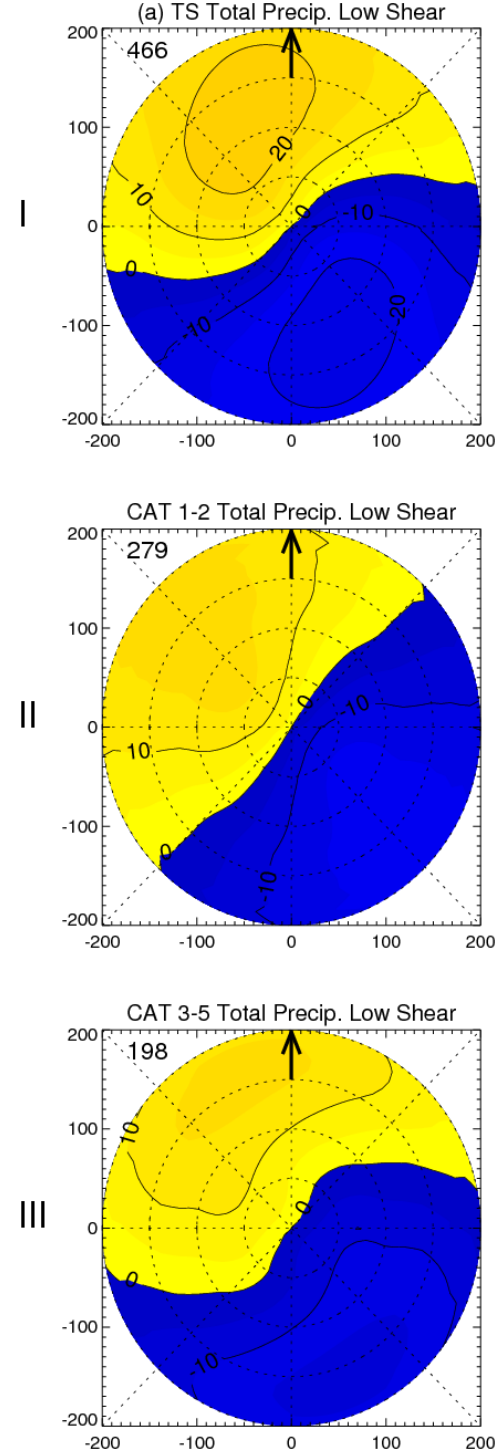
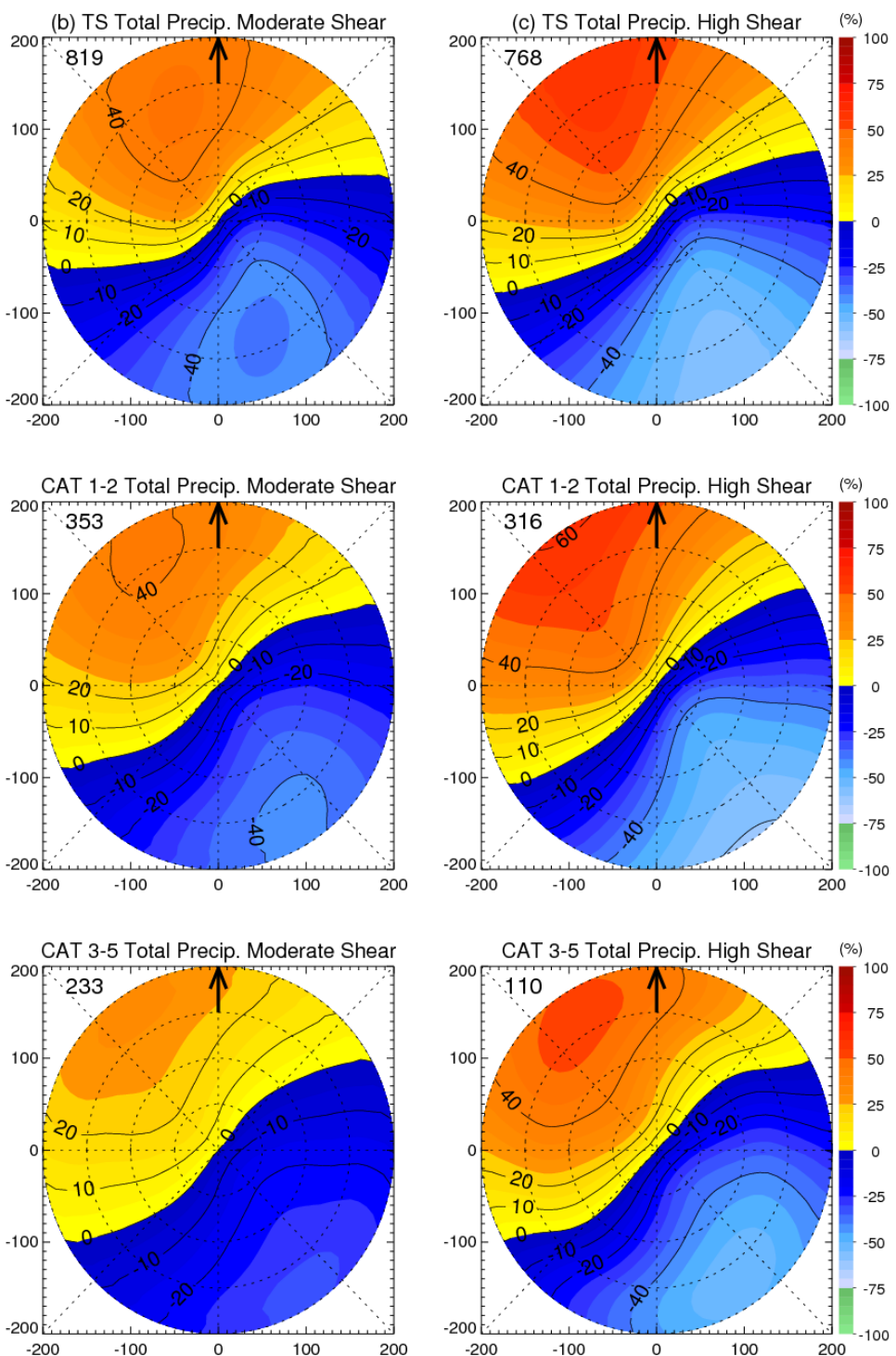

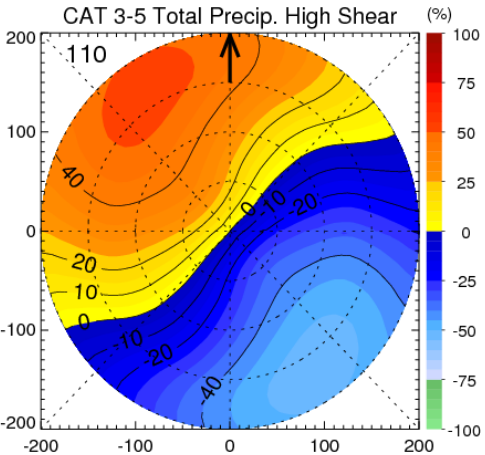

Figure 2.11: The composite shear-relative $\mathrm{R}_{1} / \mathrm{R}_{0}$ of total precipitation for (Row I) TS, (Row II) CAT 1-2, and (Row III) CAT 3-5 with [three columns] (a) low environment wind shear, (b) moderate environment wind shear, (c) high environment wind shear. The black arrow (pointing upward) represents the orientation of motion vector. The color scale represents average perturbation of the sum of the wavenumber 1 or 1-6 asymmetry components. Dotted range rings represent the 50-, 100-, 150-, and 200-km radii. 
high shear. The asymmetry index of $R_{1} / R_{0}$ for high-sheared CAT 1-2 is higher than that of high-sheared TS.

Table 2.8 (for Fig. 11 and 12) The asymmetry index of the composite shear-relative $\mathrm{R}_{1} / \mathrm{R}_{0}$ and $\mathrm{R}_{1-6} / \mathrm{R}_{0}$ of total precipitation for (Row I) TS, (Row II) CAT 1-2, and (Row III) CAT 3-5 with [three columns] (a) low environment wind shear, (b) moderate environment wind shear, (c) high environment wind shear. The percentage in each pair of parentheses represents the percentage increase of asymmetry index from $R_{1} / R_{0}$ to $R_{1-6} / R_{0}$.

\begin{tabular}{l|ccc}
\hline \hline Max. $R_{1} / R_{0}(\%)$ & Low & Moderate & High \\
\hline TS & 24.9 & 46.8 & 57.9 \\
CAT 1-2 & 18.2 & 42.5 & 61 \\
CAT 3-5 & 17.2 & 32.2 & 54 \\
\hline & \multicolumn{3}{|c}{} \\
\hline \hline Max. $R_{1-6} / R_{0}(\%)$ & Low & Moderate & High \\
\hline TS & $29.5(18 \%)$ & $79.5(70 \%)$ & $93.9(62 \%)$ \\
CAT 1-2 & $28.2(55 \%)$ & $60.5(42 \%)$ & $103.9(70 \%)$ \\
CAT 3-5 & $24.8(44 \%)$ & $42.2(31 \%)$ & $90.4(67 \%)$ \\
\hline
\end{tabular}

The shear-relative composite of $R_{1-6} / R_{0}$ is shown in Fig. 2.12. By adding the wavenumber 2-6 to wavenumber 1 , the increases of $R_{1-6} / R_{0}$ asymmetry indexes from $R_{1} / R_{0}$ of TS in Table 2.8 (corresponding to Row I of Fig. 2.12) ranks from low to high as for low (18\%), high (62\%), and moderate shear (70\%). The increases of $R_{1-6} / R_{0}$ asymmetry indexes from $R_{l} / R_{0}$ of CAT 1-2 in Table 2.8 (corresponding to Row II of Fig. 2.12) ranks from low to high as for moderate (55\%), low (42\%), and high shear (70\%). The same increases of CAT 3-5 in Table 2.8 (corresponding to Row III of Fig. 2.12) ranks from low to high as for moderate (31\%), low (44\%), and high shear (67\%). In general, the vertical wind shear magnitude higher than $10 \mathrm{~m} / \mathrm{s}$ generates more high-wavenumber energy. The phase maximum stays downshear-left after including the perturbations from wavenumber 2-6. 
However, the rainfall distribution is more localized and detailed. Lonfat et al. (2007) introduced Parametric Hurricane Rainfall Model (PHRaM), which fails at producing strong rainfall asymmetries caused by vertical shear. Possibly, it is because the model is derived from the average statistical satellite observation. However, it is also likely a result of only incorporating first and second order (without higher orders) rainfall asymmetry using Fourier decomposition in PHRaM. Adding the higher order perturbation localizes the precipitation area, which would possibly provide more accurate precipitation forecasts or simulations. 
(a) TS Total Precip. Low Shear
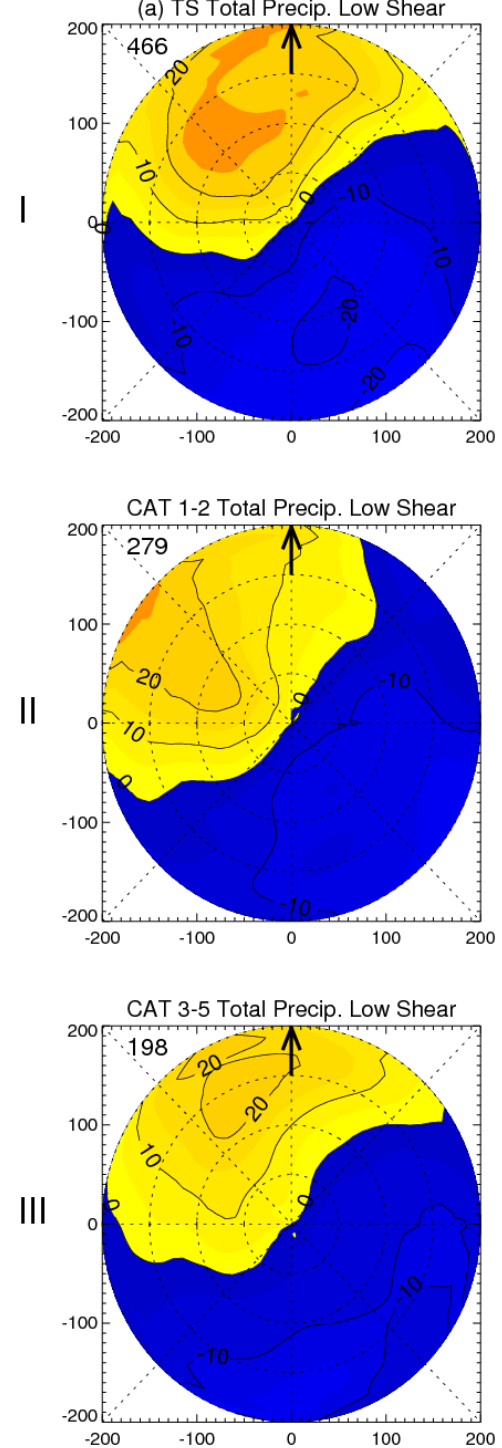
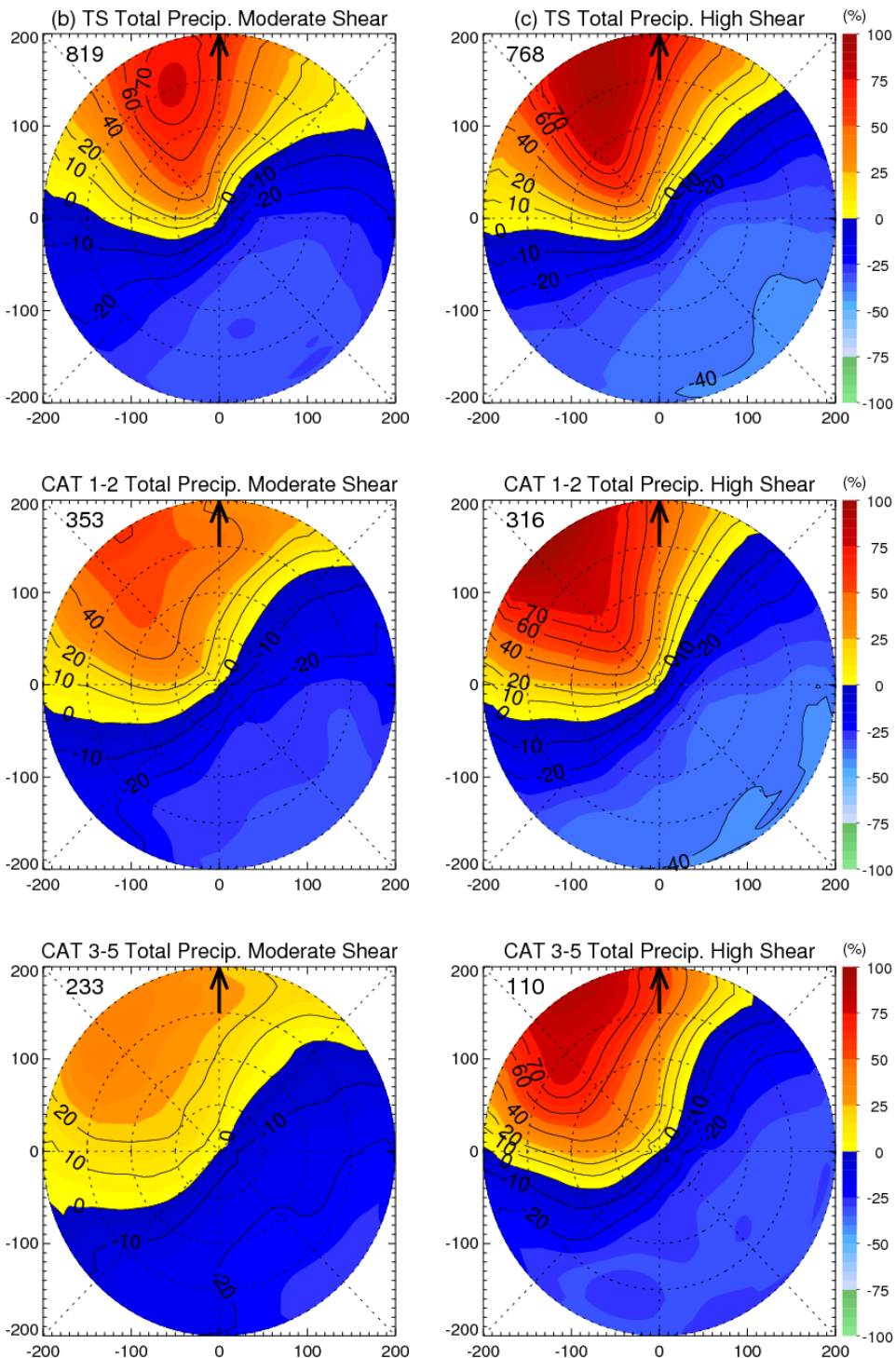

Figure 2.12: Same as FIG. 2.11, but for $\mathrm{R}_{1-6} / \mathrm{R}_{0}$

Using the 2-D composites of $R_{1-6} / R_{0}\left(R_{1} / R_{0}\right)$ of each TC overpasses binning in different vertical wind shear magnitudes, a shear-relative wavenumber-based asymmetry index is defined as the maximum $R_{1-6} / R_{0}\left(R_{1} / R_{0}\right)$ composite for wavenumber 1-6 (wavenumber 1). Figure 2.13 shows wavenumber 1 and wavenumber 1-6 asymmetry indices as a function of TC vertical wind shear magnitude for the three intensity categories 
and six basins. Figure 2.13a shows that as the shear magnitude increases, the asymmetry index of wavenumber 1-6 also increase accordingly. Among the three intensity categories, TS has the largest value of asymmetry index of wavenumber 1-6 when shear magnitude is lower than $11 \mathrm{~m} \mathrm{~s}^{-1}$. When the shear magnitude is greater than $11 \mathrm{~m} \mathrm{~s}^{-1}$, CAT 1-2 has the largest asymmetry index. The asymmetry index of CAT 3-5 exceeds that of TS after wind shear is higher than $14 \mathrm{~m} \mathrm{~s}^{-1}$. From 0 to $14 \mathrm{~m} \mathrm{~s}^{-1}$ shear magnitude, the asymmetry index of wavenumber 1-6 of the three intensity groups and six basins increases from $\sim 25 \%$ to $\sim 100 \%$. The increase caused by stronger shear magnitude is more significant than the increase caused by faster motion speed. The shear-relative asymmetry index of wavenumber 1-6 is generally 1.5 2 times higher than wavenumber 1 . As the wind shear getting stronger, the difference between asymmetry index of wavenumber 1-6 and wavenumber 1 gets larger. It confirms that higher shear generates more high-wavenumber energy. Among all the basins, in Fig. 2.13b the asymmetry index of NIO shows the largest when shear is lower than $6 \mathrm{~m} / \mathrm{s}$. the asymmetry index of $R_{1-6} / R_{0}$ for NIO does not correlate with shear magnitude well. Except for NIO, all other basins show an increase of asymmetry index for both wavenumber 1 and 1-6 as the shear magnitude increased. The wavenumber 1-6 asymmetry indexes of EPA are the highest among each shear magnitude bin from 4 to $15 \mathrm{~m} / \mathrm{s}$. However, in Table 7, the wavenumber 1-6 asymmetry index is not the largest among all the basins. It could be explained that the location of maximum TC rainfall in EPA is well impacted by wind shear; however, when adding up the perturbation field of all the composite in each shear magnitude bin, the maxima may not be at the same locations. Except for EPA and NIO, the wavenumber 1-6 asymmetry indexes of SIO and SPA are 
generally higher than those of ATL and NWP, which shows consistency with the asymmetry indexes in Table 2.7 .
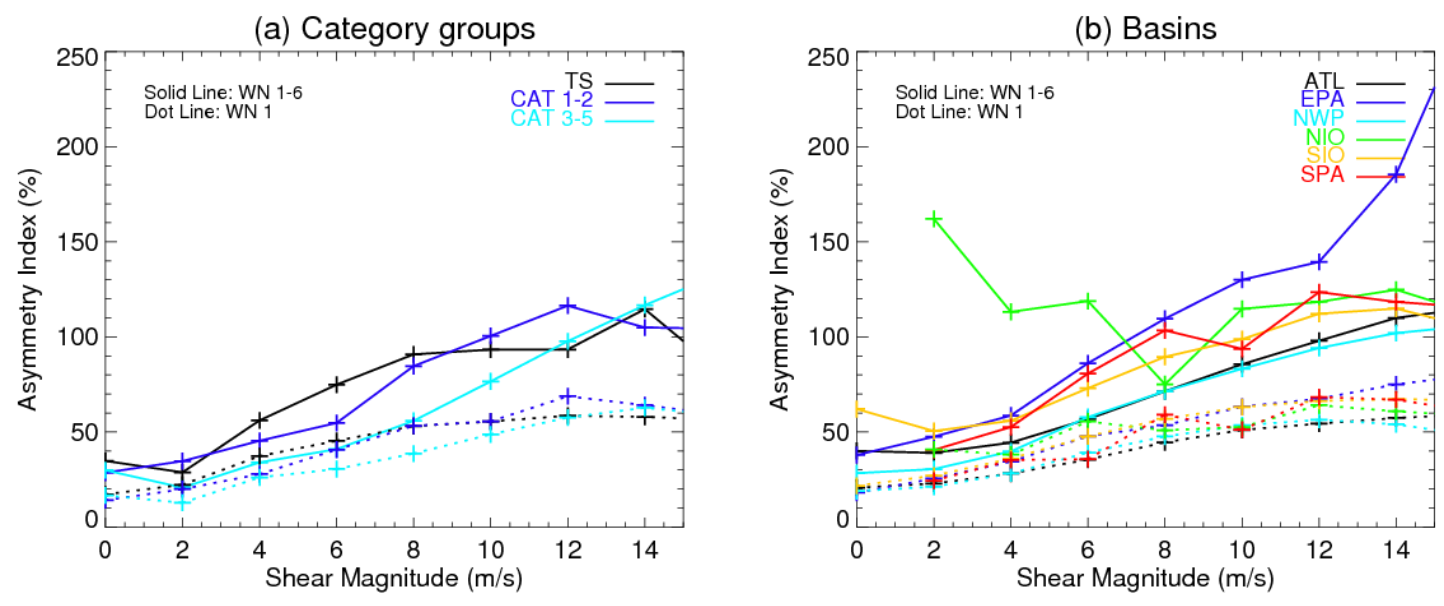

Figure 2.13: Same as FIG. 2.8, but for shear-relative wavenumber-based Asymmetry Index (integrated from 0 to $200 \mathrm{~km}$ radius) of total precipitation as a function of shear magnitude.

\subsubsection{Motion and shear combined effect}

As CKM06, the asymmetries of precipitation are examined for the combined effects of storm motion and vertical wind shear. Similar to their methodology, the samples are grouped for shear directions that are in the same, opposite, right, and left to the motion directions. However, since we now have a larger sample size than CKM06, it allows us to consider not only the shear and motion direction but the shear strength and motion speed combined effects by also stratifying the TC dataset into slow- and fast-moving speed, low, moderate, and higher shear subsets (24 subsets as shown in Fig. 2.14). Our results indicate that the shear-relative maximum $R_{l} / R_{0}$ is located in the downshear left (downshear right in $\mathrm{SH})$ 

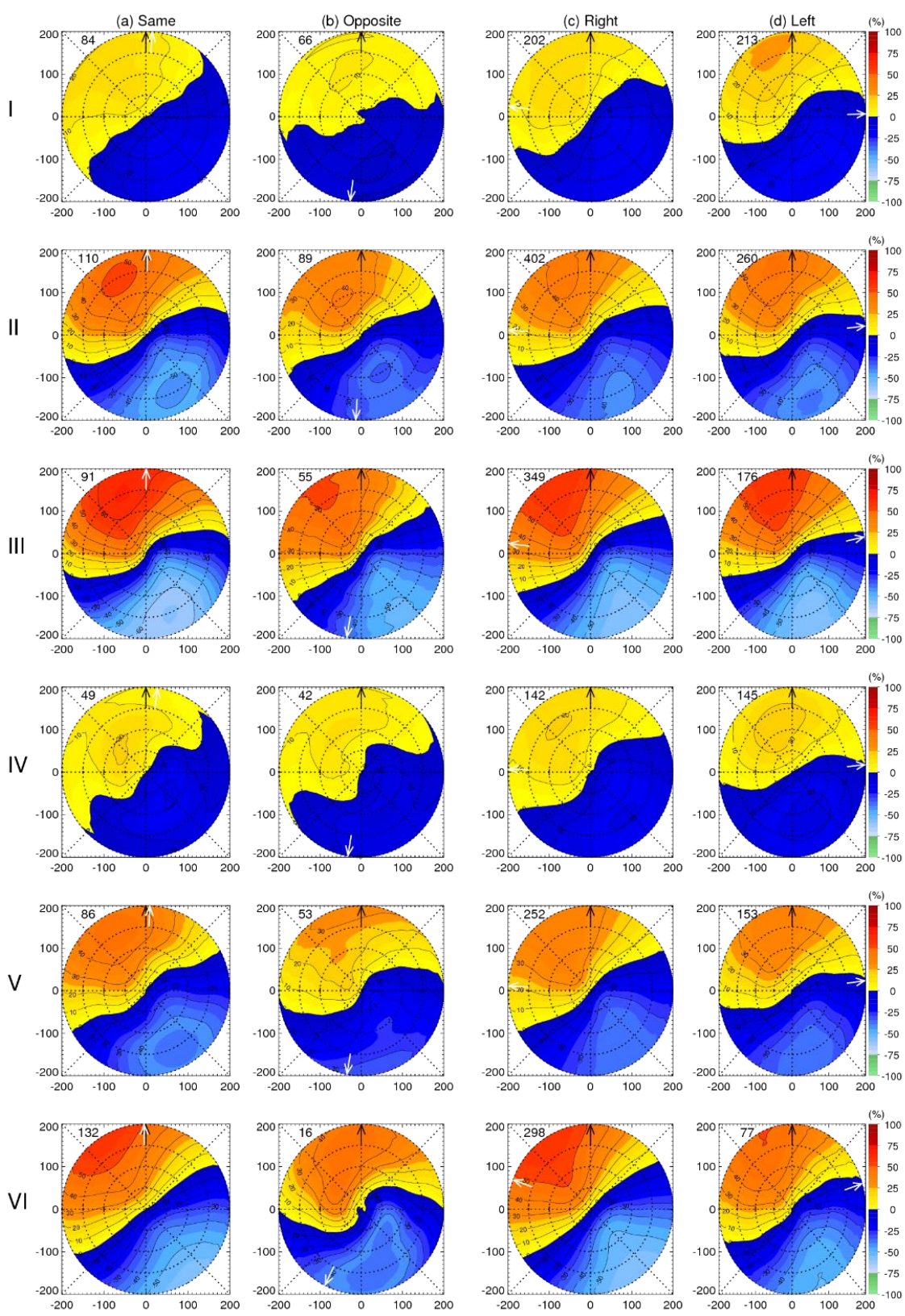

Figure 2.14: The composite shear-relative $R_{1} / R_{0}$ of total precipitation with the shear direction (a) same as, (b) opposite to, (c) to the right of, and (d) to the left of the motion direction for (I) slow motion and low shear, (II) slow motion and moderate shear, (III) slow motion and high shear, (IV) fast motion and low shear, (V) fast motion and moderate shear, and (VI) fast motion and high shear conditions. The black arrow (pointing upward) represents the orientation of vertical wind shear vector. The white arrow represents the mean orientation of motion vector. The color scale represents average perturbation of the sum of the wavenumber 1-6 asymmetry components. Dotted range rings represent the 50, 100-, 150-, and 200-km radii. 
quadrant, no matter which direction the motion vector points to (Fig. 2.14). Our results showing the TC rainfall perturbation composite with more ways of shear and motion combinations are consistent with Wingo and Cecil (2010), Corbosiero and Molinari (2002), and Corbosiero and Molinari (2003), which shows in both strong and weak shear environment, precipitation is favored downshear left. Table 2.9 shows that the asymmetry index of $R_{l} / R_{0}$ is generally the highest for the group with motion and shear to the same direction (Fig. 2.14 column (a)) and lowest for the group with motion and shear to the opposite direction (Fig. 2.14 column (b)). In Table 2.9, it also shows that the sub-category of slow motion and high shear with motion and shear to the same direction is the most asymmetric (the asymmetry index is $66.2 \%$ ). The sub-category of slow motion and low shear with motion and shear to the different direction is the least asymmetric (the asymmetry is $12.1 \%$ ). Figure 2.15 shows the same composite with adding wavenumber 26 components. The maximum $R_{1-6} / R_{0}$ is mostly downshear left except for low shear cases (Fig. $2.15 \mathrm{I}, \mathrm{IV}$ ). For the low shear composite, the positive perturbation is not evenly concentrated in downshear left region but also distributed in other quadrants. The most noticeable change is Fig. $2.15 \mathrm{IVd}$. By adding wavenumber $2-6$, the maximum $R_{1-6} / R_{0}$ is downshear right. It indicates when the shear magnitude is low, the effects from storm motion dominate more on the rainfall distribution. Table 2.9 also shows that after adding wavenumber 2-6 perturbation to wavenumber 1 , the percentage generally increases the most for the high shear cases in Row III and VI. It confirms with Fig. 2.12 with more subcategories that the vertical wind shear magnitude higher than $10 \mathrm{~m} / \mathrm{s}$ generates more highwavenumber energy. 

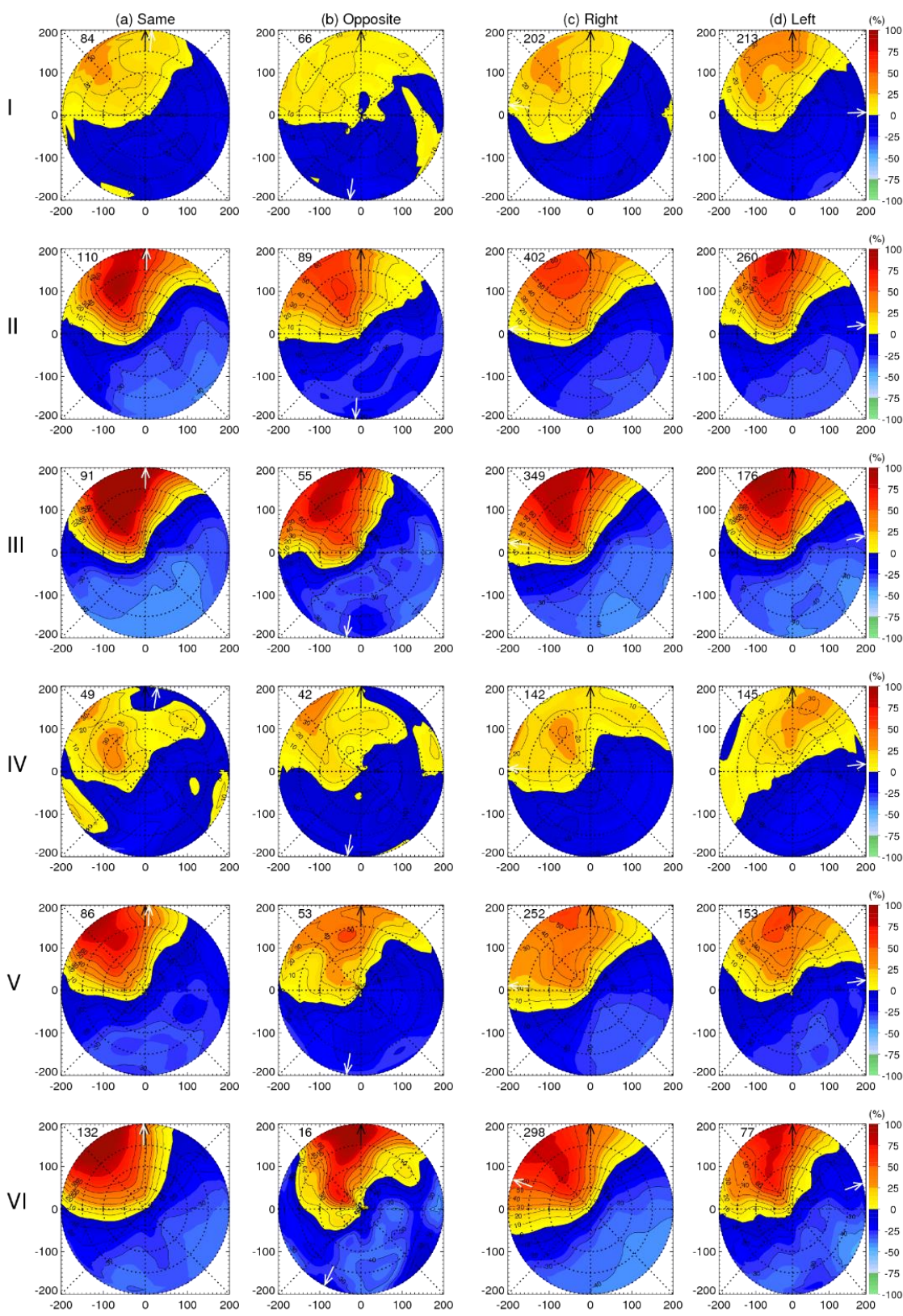

Figure 2.15: Same as FIG. 2.14, but for the composite shear-relative $R_{1-6} / R_{0}$ of total precipitation. 
Table 2.9 (for Fig. 14 and 15) The asymmetry index of the composite shear-relative $R_{1} / R_{0}$ and $\mathrm{R}_{1-6} / \mathrm{R}_{0}$ of total precipitation with the shear direction (a) same as, (b) opposite to, (c) to the right of, and (d) to the left of the motion direction for (I) slow motion and low shear, (II) slow motion and moderate shear, (III) slow motion and high shear, (IV) fast motion and low shear, (V) fast motion and moderate shear, and (VI) fast motion and high shear conditions. The percentage in each pair of parentheses represents the percentage increase of asymmetry index from $R_{1} / R_{0}$ to $R_{1-6} / R_{0}$.

\begin{tabular}{l|cccc}
\hline \hline Max. $R_{l} / R_{0}$ & (a) Same & (b) Opposite & (c) Right & (d) Left \\
\hline I & 21.1 & 12.1 & 20.5 & 28 \\
II & 54 & 43 & 41.9 & 46.7 \\
III & 66.2 & 55.2 & 59.3 & 59.2 \\
IV & 21.9 & 18.9 & 21.7 & 24.8 \\
V & 49.1 & 35.4 & 40 & 39.9 \\
VI & 58.1 & 52.1 & 59.2 & 50.5 \\
\hline & \multicolumn{4}{|c}{} \\
\hline \hline Max. $R_{I-6} / R_{0}$ & (a) Same & (b) Opposite & (c) Right & (d) Left \\
\hline I & $33.4(58 \%)$ & $21(74 \%)$ & $31.1(52 \%)$ & $39(39 \%)$ \\
II & $104(93 \%)$ & $64.9(51 \%)$ & $61.9(48 \%)$ & $80.9(73 \%)$ \\
III & $139.8(111 \%)$ & $98.8(79 \%)$ & $90.3(52 \%)$ & $104.6(77 \%)$ \\
IV & $41.8(91 \%)$ & $41.7(121 \%)$ & $34.2(58 \%)$ & $32.9(33 \%)$ \\
V & $96.6(97 \%)$ & $58.2(64 \%)$ & $56.2(41 \%)$ & $59.6(49 \%)$ \\
VI & $117.6(102 \%)$ & $113.3(117 \%)$ & $81.9(38 \%)$ & $87.8(74 \%)$ \\
\hline
\end{tabular}

Though the shear relative composite in Fig. 2.14 and 2.15 shows their significance relative to wind shear, it is still unclear how the maximum asymmetry would be distributed if it is composited relative to motion direction. In Fig. 2.16, the composites of the same sub-categories as in Fig. 2.14 and 2.15 are shown, but all the composites are rotated by motion direction. It shows that the maximum $R_{l} / R_{0}$ is most downshear left. It is worth noting that the shear direction is calculated by averaging the shear direction of all the 
samples, which is not necessary the wind shear direction of every TC in the sample. So, in column (a) of Fig. 2.16, the down-motion location is an approximate downshear. For column (b) of Fig. 2.16, the maximum $R_{I} / R_{0}$ is mainly up-motion right, which corresponds to an approximate downshear right. For column (c), the maximum $R_{I} / R_{0}$ is in down-motion right corresponding to an approximate downshear right. For column (d) the maximum $R_{l} / R$ is in the left of motion corresponding to an approximate downshear left except for Fig. 2.16IVd, which the maximum is mainly downshear. The location of motion-relative maximum $R_{l} / R_{0}$ (Fig. 2.16) is highly dependent on the direction of vertical wind shear direction. As it is seen that the distribution of positive $R_{l} / R_{0}$ is mostly downshear-left, except for the composite with fast motion and low shear (Fig. 2.16 IVd). After adding wavenumber 2-6, the composite of $R_{1-6} / R_{0}$ for both Fig. 2.17IVd and Fig. 2.17Id are not downshear left. Instead, the maxima are downshear (down-motion-left). It shows consistency with Fig. 2.15IVd and Fig 2.15Id, which in both panels the maximum $R_{1-6} / R_{0}$ is also downshear. 

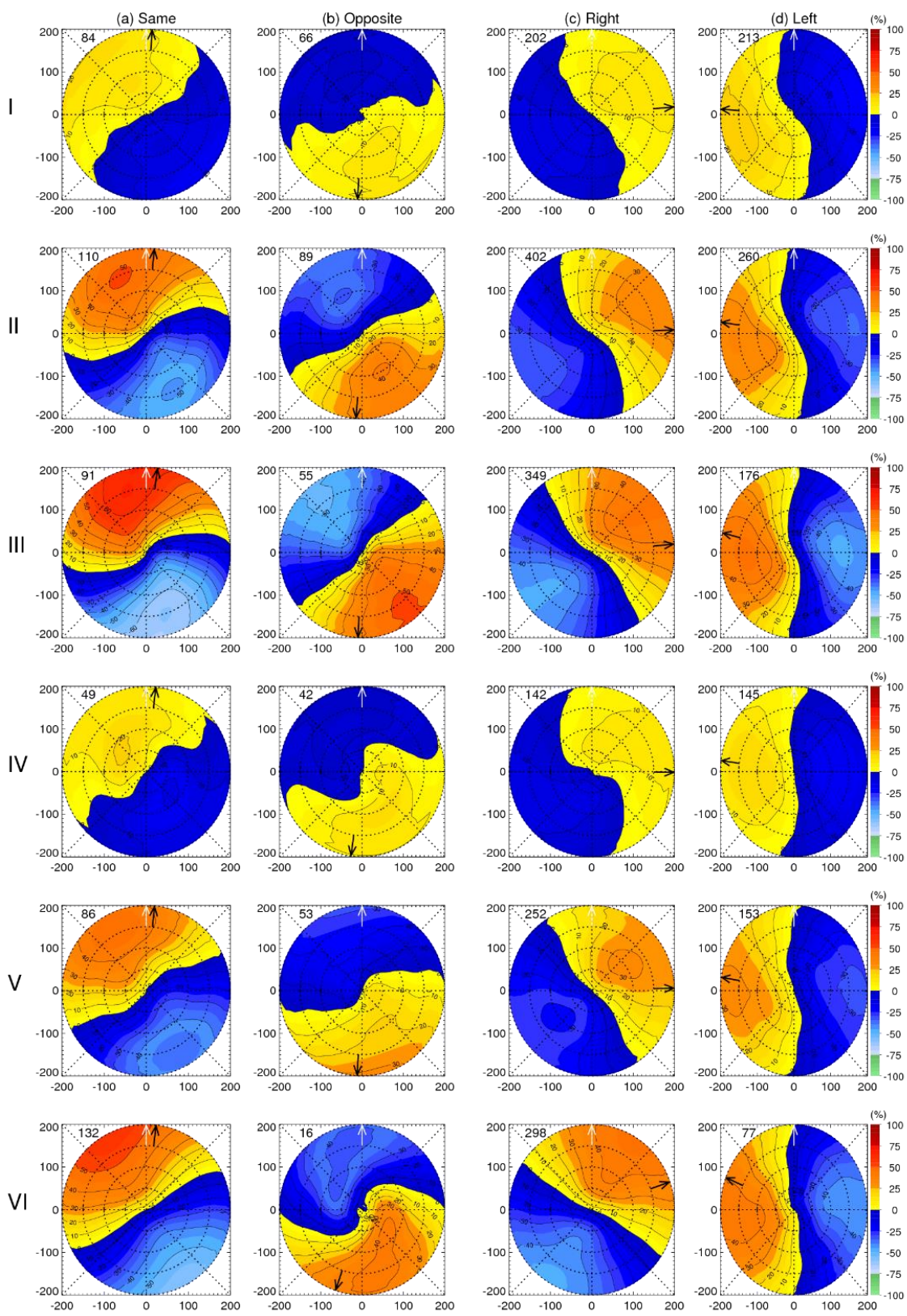

Figure 2.16: The composite motion-relative sum of $\mathrm{R}_{1} / \mathrm{R}_{0}$ of total precipitation with the shear direction (a) same as, (b) opposite to, (c) to the right of, and (d) to the left of the motion direction for (I) slow motion and low shear, (II) slow motion and moderate shear, (III) slow motion and high shear, (IV) fast motion and low shear, (V) fast motion and moderate shear, and (VI) fast motion and high shear conditions. The black arrow represents the orientation of vertical wind shear vector. The white arrow (pointing upward) represents the mean orientation of motion vector. The color scale represents the average perturbation of the sum of the wavenumber 1-6 asymmetry components. Dotted range rings represent the 50-, 100-, 150-, and 200-km radii. 

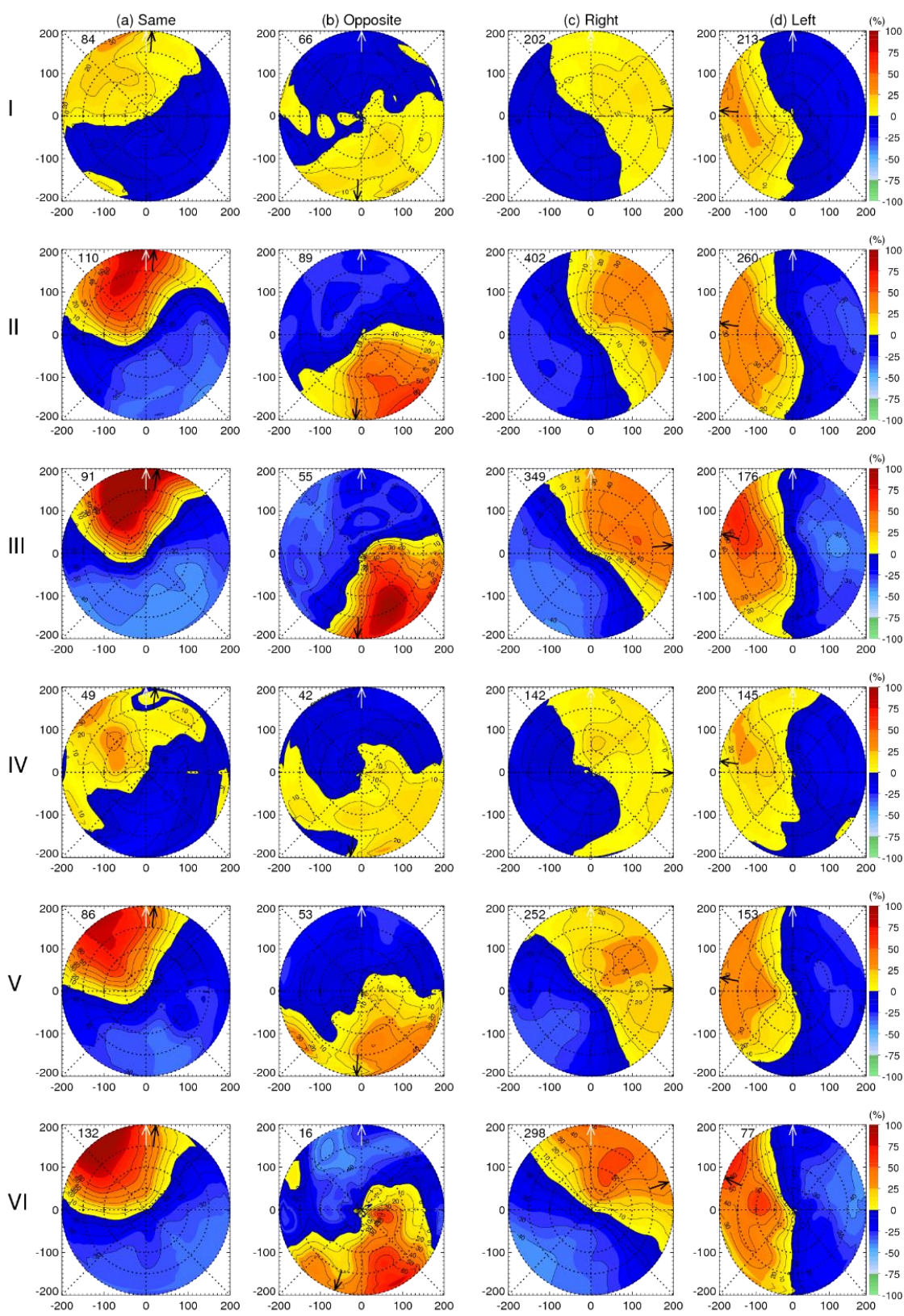

Figure 2.17: Same as FIG. 2.16, but for the composite motion-relative $\mathrm{R}_{1-6} / \mathrm{R}_{0}$ of total precipitation. 


\subsection{Conclusions}

Sixteen years of TRMM TMI retrieved rain rate data are employed to demonstrate the Fourier low- and high- wavenumber asymmetries of four types of precipitation. From the energy budget perspective, wavenumber 1 is dominant in all types of precipitation. Wavenumber 1-6 predominantly contributes to the total energy. Moderate and heavy precipitation have relatively less contribution to total energy from low-wavenumbers $(\leq 6)$ than light precipitation. Radially inside $100 \mathrm{~km}$ radius of the $\mathrm{TC}$ inner core region, moderate and heavy precipitation are more responsible for the precipitation asymmetry. Compared with light and moderate precipitation, the heavy rainfall contributes the most to the total wavenumber 1-6 asymmetry. The asymmetry in the precipitation distribution is mostly determined by the heaviest precipitation.

In the energy spectrum:

- Wavenumber 2-6 contributes about the same amount of energy as wavenumber 1 .

In both a motion-relative and shear-relative framework:

- Adding wavenumbers 2-6 perturbation to wavenumber 1, the amplitude increases more for fast-moving and high sheared TCs.

- By adding the higher wavenumber asymmetry component, the location of dominant precipitating region relative to motion shifts cyclonically. The precipitation region relative to shear does not change significantly.

- As the motion speed increases, the degree of asymmetry increases a small amount. As the shear magnitude increases, there is a noticeable large increase in the degree of asymmetry.

- As the speed of motion increases, the asymmetric precipitation shifts from down- 
motion to down-motion-left region. With shear magnitude increasing, the maximum asymmetry location converges at downshear left. When shear magnitude is low, the maximum asymmetry of TS is more towards downshear in TC outer region.

Under the combined effects from shear and motion:

- With an exception for when the vertical wind shear is weak, the precipitation distribution is predominantly affected by vertical wind shear rather than storm motion.

The maximum wavenumber 1-6 total precipitation asymmetries are in general displaced down-motion left (right) in $\mathrm{NH}(\mathrm{SH})$, which is consistent with the nonlinear model study of Kepert and Wang [2001]. The maximum wavenumber 1 precipitation asymmetry shifts from the down-motion left to right with increasing intensity. Wavenumbers 2-6 also contribute to the magnitude of precipitation asymmetry, and the location of the precipitation asymmetry in ATL, EPA, and SPA. In these three basins, when the wavenumbers 2-6 is added, the maximum asymmetry rotates from down-motion to down-motion-left. Compared with Shapiro [1983], result in this paper confirms Kepert and Wang [2001] that the nonlinear spectrum will bring a cyclonic rotation of the maximum precipitation asymmetry to the linear result.

The shear-relative precipitation asymmetry is generally displaced downshear-left (right) in the $\mathrm{NH}(\mathrm{SH})$. Vertical wind shear is generally more responsible for the precipitation distribution (and subsequent asymmetry) than the effects from storm motion. In different shear environments, the amplitude of rainfall asymmetry increases as the wind 
shear magnitude gets stronger. No matter how the magnitude of shear changes, the maximum asymmetry is located downshear-left.

The combined effects of both shear and motion show the magnitude of asymmetry is mainly determined by the vertical wind shear magnitude. But when shear is weak, the precipitation distribution (and subsequent asymmetry) is determined more by storm motion. The main contributors to creating precipitation asymmetry, and its maximum location, are moderate and heavy precipitation. By incorporating the energy from wavenumber 2-6 into wavenumber 1 , it localizes the maximum precipitation asymmetry. 


\section{QUANTIFICATION OF SHEAR-RELATIVE PRECIPITATION}

\section{ASYMMETRIES OF TROPICAL CYCLONES IN DIFFERENT INTENSITY \\ CHANGES STAGES}

\subsection{Abstract}

Tropical cyclone (TC) precipitation asymmetry represented by Fourier decomposed perturbation normalized by the azimuthal mean rain rate are compiled from 16-yr Tropical Rainfall Measuring Mission (TRMM) Mircowave Imager (TMI) 2A12 rain rate. Firstly, the precipitation asymmetry properties of rapid intensification (RI) and non-RI storms are examined. The dataset of 2,186 global tropical storms through category 2 hurricanes is divided by future $24-\mathrm{h}$ intensity change and exclusively includes storms with at least moderately favorable environmental conditions. TMI $2 \mathrm{~A} 12$ rain rate $(\mathrm{R})$ is categorized into four precipitation types: total precipitation $\left(\mathrm{R} \geq 0.5 \mathrm{~mm} \mathrm{hr}^{-1}\right)$, heavy precipitation $(\mathrm{R} \geq 10$ $\mathrm{mm} \mathrm{hr}{ }^{-1}$ ), moderate precipitation $\left(10 \mathrm{~mm} \mathrm{hr}^{-1}>\mathrm{R} \geq 5 \mathrm{~mm} \mathrm{hr}^{-1}\right)$, and light precipitation (5 $\mathrm{mm} \mathrm{hr}{ }^{-1}>\mathrm{R} \geq 0.5 \mathrm{~mm} \mathrm{hr}^{-1}$ ). The normalized wavenumber 1-6 asymmetry, indicates quantitatively that in favorable environmental conditions, the lower asymmetry of light precipitation is most strongly correlated with future intensity change. For all four types of precipitation, non-RI storms are generally more asymmetric than RI storms. The asymmetry of heavy precipitation is higher than the other three types of precipitation in RI storms, which is considered as a necessary condition for storm to intensify. Secondly, the 595 sampled overpasses are classified into 14 categories in the timeline of an RI event from 48 hours before RI until RI ends. The decrease of normalized wavenumber 1-6 asymmetries in the inner core region of all four types of precipitation several hours before RI onset was quantitatively demonstrated to be critical for TC RI. 


\subsection{Introduction}

Predicting tropical cyclone (TC) intensification, especially rapid intensification (RI), is challenging for forecasters. Favorable environmental conditions have been deemed as the necessary condition for RI (Hendricks et al. 2010; Kaplan et al. 2010), but the sufficient condition is still unknown. Recent studies have indicated the urge of understanding the storm's internal structure and dynamics in order to pinpoint the exact onset, duration, and magnitude of the RI event.

The distribution of precipitation and convection has been linked with storm intensity and intensification. There has been a debate on whether the asymmetric deep convection or the widespread, symmetric shallow-to-moderate precipitation is more important to TC intensification and RI. One side of the debate emphasizes the importance of asymmetric deep convection such as hot towers and convective bursts (Hendricks et al. 2004; Kelley et al. 2004; Montgomery et al. 2006; Nguyen et al. 2008; Montgomery and Smith 2011; Nguyen and Molinari 2012; Rogers et al. 2015). However, the other side of the debate emphasizes the symmetric mechanism for TC intensification. Many early theoretical studies have shown that a TC intensifies through an axisymmetric mechanism (Ooyama 1969; Shapiro and Willoughby 1982). Nolan et al. (2007) have argued that the azimuthally averaged latent heating release is much more important for the vortex intensification than asymmetric heating.

Several recent satellite-based statistical studies supported the symmetric mechanism of TC intensification with a focus on RI. Jiang (2012) examined the inner-core convection in different TC intensity change categories and found that hot towers were 
neither a necessary nor sufficient condition for RI. A symmetric ring pattern in $37 \mathrm{GHz}$ composite passive microwave images was found as a good indicator of TC RI (Kieper and Jiang 2012). Using a 15-yr multiplatform dataset of passive microwave satellite data, Alvey et al. (2015) demonstrated that the rate of symmetrization prior to and during intensification increased with increasing intensification rate. Statistical studies as Jiang (2012), Jiang and Ramirez (2013), and Alvey et al. (2015) advanced the understanding of the properties of storms that undergo RI by comparing RI cases with other intensity change categories (slowly intensifying, neutral, or weakening categories). Using the differences between RI and non-RI storms, it is helpful for the 24-h future intensity forecast; however, this approach also has limitations on understanding the storm evolution. Since RI happens as an event as TC evolving (Kieper and Jiang 2012). Each event could last 48-60 hrs. By only comparing the differences between RI and non-RI storms, it does not distinguish between stages of an RI event, including before RI onset, at or around RI onset, during the middle (or continuing stage) of an RI event, and the ending period of an RI event.

The satellite-based studies of Zagrodinik and Jiang (2014, hereafter ZJ14) and Tao and Jiang (2015, hereafter TJ15) begun approaching RI as an event by defining the onset of RI as the starting time of each RI event instead of that of each 24-h RI period. They both separated RI cases into two subcategories RI initial and RI continuing. RI initial is defined as overpasses that occurred within $12 \mathrm{~h}$ of an RI onset. RI continuing is defined as overpasses that occurred during a period in which the storm had a previous 24-h RI case beginning at 12,18 , or $24 \mathrm{~h}$ prior to the overpass. The RI event in both subcategories continued for at least $24 \mathrm{~h}$ following the overpass as well. Using 14 years of Tropical Rainfall Measuring Mission (TRMM) Precipitation Radar (PR) data for Norther 
hemisphere TCs, ZJ14 demonstrated that the distributions of TC rainfall and latent heating were more symmetric at the beginning of RI and became more symmetric during the middle of RI events. Using the same dataset extended to global TCs, TJ15 analyzed the distributions of four types of precipitation-convection in different storm intensity change categories. They found that increased and widespread shallow precipitation is the first sign of RI. A ring of almost $90 \%$ occurrence of total precipitation was found for storms in the middle of RI. Both studies were able to demonstrate the evolution of precipitation and convection distributions during RI events from the beginning to the middle of RI. Tao et al. (2017) further expanded the previous event-based approach by incorporating the analysis for the period before RI onset and during the ending period of RI events [defined as less than 24h before the ending time of an RI event; cf. Fig. 13 of Tao and Jiang (2015)].

However, none of the above-mentioned satellite-based studies quantified the magnitude of asymmetry in a sophisticated way. Kieper and Jiang (2012) determined the ring pattern subjectively by manually reviewing microwave images case by case. The precipitative and convective asymmetries were investigated qualitatively by showing the distributions relative to the shear direction in ZJ14, TJ15, and Tao et al. (2017). Alvey et al. (2015) quantified convective asymmetries through a simple symmetry index calculated by differencing the mean $85-91 \mathrm{GHz}$ polarization corrected brightness temperature (PCT, Spencer et al. 1989) for all pixels less than $250-\mathrm{K}$ in different quadrants relative to shear direction. Also, some studies (e.g.: Kelley et al. 2004; Montgomery et al. 2006; Nguyen and Molinari 2012; Rogers et al. 2015) were for TC intensification in general, not specifically for RI. In this study, we plan to quantify the precipitation asymmetry in relation to RI. 
Many previous studies examined the convective and precipitative asymmetries qualitatively in TCs by showing the shear-relative or coastline-relative distributions of rainfall and convective parameters (Corbosiero and Monlinari 2002; Chan et al. 2004; Ueno 2008; Wingo and Cecil 2010). Corbosiero and Molinari (2002) using the flash locations data from National Lightning Detection Network (NLDN) showed an asymmetric convection preference of downshear left occurring in the storm core and a strong preference of downshear right in the outer rain bands. In their study, convective asymmetries were examined by comparing the number of lightning counts in different shear-relative quadrants or octants. Chan et al. (2004) used the average cloud-top temperature in four different sectors (Northward, Eastward, Southward, and Westward) of TCs relative to the coastline to illustrate the convective asymmetries. Ueno (2008) evaluated the simulated rainfall asymmetry by comparing the number of hours of the highest hourly rain rate in different octants relative to vertical wind shear direction. Wingo and Cecil (2010) analyzed the global rainfall distributions in TCs based on satellite data derived from passive microwave radiometers. Their results showed the relationships between the TC intensity, the geographical location, the shear magnitude, the rainfall magnitude and the rainfall asymmetry relative to the shear direction.

The Fourier decomposition method has been shown as a formal, sophisticated, and effective way to quantify rainfall and convective asymmetries of TCs. Using a first-order Fourier decomposition for three years of TRMM Microwave Imager (TMI) 2A12 rainfall data, Lonfat et al. (2004) studied the rainfall asymmetry relative to storm motion for TCs in different basins and different intensity stages. They found that the maximum wavenumber-1 rainfall asymmetry was located in the front quadrant relative to the storm 
motion direction and higher intensity storms had less asymmetric rainfall distributions. Using the same TMI dataset and methodology, Chen et al. (2006) analyzed the combined effects of vertical wind shear and storm motion on the rainfall asymmetry of TCs in different basins and different storm intensity stages. They showed that the predominant wavenumber-1 rainfall asymmetry maximum was downshear left (downshear right for Southern Hemisphere) in a high shear environment and the inner core precipitation distribution was affected more by the storm motion in a low shear environment. In both Wu et al. (2013) and Yu et al. (2015), a Fourier decomposition was applied to radar reflectivity and satellite derived rainfall data respectively to evaluate the asymmetric components of rainfall of TCs making landfall over China.

The primary goal of this study is to quantify the rainfall asymmetry relative to the vertical wind shear direction for TCs in different intensity change stages, with an emphasis on comparing the degree of rainfall asymmetry in RI storms with that in non-RI storms and examine the evolution of quantified precipitation asymmetries during the entire life cycle of RI events. Unlike ZJ14 and TJ15 which examined the precipitation and convection asymmetry qualitatively using TRMM PR reflectivities, this study applies a Fourier decomposition to 16-yr TRMM TMI 2A12 rainfall estimates to calculate the lower wavenumber asymmetry of rainfall. With the advantage that the TMI has a wider swath than the PR, a larger sample size and wider storm coverage are achieved. Wingo and Cecil (2010) compared the asymmetry of mean rain rate with heavy rain and found that heavy rain is comparatively rare in the upshear quadrants. In this study, we separate precipitation into four types including total precipitation, light precipitation, moderate precipitation, and 
heavy precipitation to examine how the asymmetry of different types of precipitation changes in RI storms versus non-RI storms and during the life cycle of RI events.

\subsection{Data and Method}

\subsubsection{TRMM overpass selection}

The dataset for this study is derived from 16 years of TRMM TMI (Kummerow et al. 1998) overpasses from 1998 to 2013. Using the TRMM Tropical Cyclone Precipitation Feature (TCPF) database (Jiang et al. 2011) each TC that reached tropical storm intensity during its lifetime observed by TRMM satellite is included. The storm center, intensity and storm translation speed of each overpass are obtained by interpolating from global TC besttrack. The TMI has an 878-km swath (760 km before August 2001 orbital boost), which provides good coverage observations of TC inner cores and rainbands. Compared with the previous studies (ZJ14 and TJ15) which used the TRMM precipitation radar (PR) data, this study using TRMM TMI data. It allows us to have larger dataset because of the broader swath width of TMI sensor. To focus on how inner core convection distribution affects RI, environmental criteria have been applied to select the TMI overpasses (ZJ14 and TJ15). The environmental criteria include: 1) Sea Surface Temperature (SST) $\left.>26^{\circ} \mathrm{C}, 2\right)$ vertical wind shear $<16 \mathrm{~m} \mathrm{~s}^{-1}, 3$ ) mean inner $250 \mathrm{~km}$ total precipitable water (TPW) $>50 \mathrm{~mm}, 4$ ) storm translation speed $<11 \mathrm{~m} \mathrm{~s}^{-1}, 5$ ) TC center located over water both at the time of the overpass and 24-h in the future, and 6) the intensity of the storm at the time of the overpass must be between tropical storm and category 2 hurricane. Since before applied the intensity and environmental condition restriction to the dataset, there are only 9 out of the 2, 935 overpasses are category 3 to 5 hurricanes that underwent RI and also the higher intensity 
storms intensify through other dynamic process (Willoughby et al. 1982). After applying the environmental condition restriction to 2,935 overpasses in both of the northern and southern hemisphere with the storm's core region $(200 \mathrm{~km}$ radius from the storm center) well-observed by the TMI sensor, there are 2,186 overpasses selected in this study as shown in Table 3.1. The storm centers interpolated from best track are manually recentered based on TMI $37 \mathrm{GHz}$ color composite image. The TMI surface rainfall estimates of version 7 (V7) (Kummerow et al. 1996, 2001) are used in this study. The TMI 2A12 rainfall algorithm (Kummerow et al. 1996, 2001) retrieves surface rainfall using TMI frequencies $(10,19,21,37$, and $85 \mathrm{GHz})$ based on the Goddard Profiling algorithm, which uses a Bayesian approach to represent surface rain. The horizontal resolution of TMI products is $5.1 * 5.1 \mathrm{~km}^{2}\left(4.4 * 4.4 \mathrm{~km}^{2}\right.$ before August 2001 orbital boost).

Table 3.1:The sample size of different intensity change categories

\begin{tabular}{ccc}
\hline \hline Category & Max. Wind Speed Range $(\mathrm{kt})$ & \# TMI overpasses \\
\hline W & $\operatorname{Vmax}(+24)-\operatorname{Vmax}(0)<-0 \mathrm{kt}$ & 399 \\
$\mathrm{~N}$ & $-10 \leq \mathrm{Vmax}(+24)-\operatorname{Vmax}(0)<10 \mathrm{kt}$ & 822 \\
$\mathrm{SI}$ & $10 \leq \mathrm{Vmax}(+24)-\mathrm{V} \max (0)<30 \mathrm{kt}$ & 694 \\
RI & $\mathrm{Vmax}(+24)-\mathrm{V} \max (0) \geq 30 \mathrm{kt}$ & 271 \\
\hline Total & & 2186 \\
\hline \hline
\end{tabular}

Environmental parameters for each overpass are included in the dataset. The sea surface temperature (SST) is obtained from Reynolds SST analyses with 0.25 degree spatial and 1-day temporal resolution (Reynolds et al. 2007). The 0.75-degree resolution total precipitable water (TPW) data from European Centre for Medium-Range Weather Forecasting (ECMWF) was averaged for the innermost $250 \mathrm{~km}$ around the TC center. The vertical wind shear magnitude and direction are calculated by taking the average of 200- 
and 850-hpa wind vectors between 500 and $750 \mathrm{~km}$ from the storm center from ECMWF (Chen et al. 2006; Hence and Houze 2011; Zagrodnik and Jiang 2014).

\subsubsection{Intensity change categories and RI event-based dataset}

The best track data from the National Hurricane Center (NHC) and Joint Typhoon Warning Center (JTWC) are used to determine intensity change categories and RI events from a total of 1,518 TCs between 1998 and 2013. The total of 2,186 TMI overpasses over global TC-prone basins are divided into four categories based on the intensity change between the time of the TRMM overpass and $24 \mathrm{~h}$ in the future. Table 3.1 shows the definitions and sample sizes for each intensity change categories. Based on the previous studies (Jiang 2012; Jiang and Ramirez 2013; ZJ14; TJ15), four intensity change categories: weakening (W), neutral (N), slowly intensifying (SI), and RI.

An RI event is defined as multiple, continuous, and overlapping 24-h periods where the maximum sustained winds of each period increased by at least $30 \mathrm{kt}$ (cf. Fig. 13 of TJ15 and Tao et al. 2017). Please also see Fig. 2 of Kieper and Jiang (2012) for an illustration of Hurricane Wilma (2005)'s RI event. Here, each 24-h period within an RI event refers to one RI case in Kaplan and DeMaria (2003). The onset of an RI event is defined as the starting time of the first 24-h RI case within the RI event. The ending time of an RI event is the ending time of the last 24-h RI case within the RI event. As defined by ZJ14, the RI initial period is within $12 \mathrm{~h}$ after RI onset, while the RI continuing period refers to the period between $12 \mathrm{~h}$ after RI onset and $24 \mathrm{~h}$ before RI ends. In the following text, we collectively call ' RI initial (RI INI)", and "RI continuing (RI CON)'” periods as "during RI.'” The RI ending period refers to the $24-\mathrm{h}$ period between $24 \mathrm{~h}$ before RI ends and the ending time of 
this RI event. Unlike RI INI and RI CON categories, which require at least $24 \mathrm{~h}$ before an RI event ends, the RI ending category has no predictive power and may correspond to the slowly intensifying (SI), neutral (N), or weakening (W) category as defined by TJ15.

Out of 1,518 global TCs between 1998 and 2013, 552 TCs underwent RI. Here, 700 RI events are identified due to some storms having more than one RI event during their lifetime. The length of an RI event is defined as the duration between when RI starts and RI ends. The minimum length is $24 \mathrm{~h}$. Within the TC samples used in this study, the maximum length of an RI event is $78 \mathrm{~h}$ and the mean length is about $38 \mathrm{~h}$. Based on the TRMM observational time and the start/end time of RI events, the selected TMI overpasses are classified into 14 categories as listed in Table 3.2. Also displayed in Table 3.2 is the number of selected TMI overpasses in various future 24-h intensity change categories, including RI, SI, N, and W. 
Table 3.2: The sample size in different TC intensity change categories for before RI, during RI and in the RI ending period.

\begin{tabular}{|c|c|c|c|c|c|c|}
\hline \multicolumn{2}{|c|}{ Category } & ALL & RI & SI & $\mathrm{N}$ & W \\
\hline \multirow[t]{5}{*}{ Before RI } & $36-48 \mathrm{~h}$ before & 26 & 0 & 14 & 10 & 2 \\
\hline & 24-36 h before & 39 & 0 & 25 & 14 & 0 \\
\hline & $18-24 \mathrm{~h}$ before & 32 & 0 & 21 & 11 & 0 \\
\hline & $12-18 \mathrm{~h}$ before & 47 & 0 & 37 & 10 & 0 \\
\hline & 6-12 h before & 44 & 0 & 42 & 2 & 0 \\
\hline \multirow[t]{5}{*}{ During RI } & 0-6 h before & 36 & 0 & 36 & 0 & 0 \\
\hline & $0-6 \mathrm{~h}$ after & 41 & 41 & 0 & 0 & 0 \\
\hline & $6-12 \mathrm{~h}$ after & 45 & 45 & 0 & 0 & 0 \\
\hline & $12-18 \mathrm{~h}$ after & 45 & 45 & 0 & 0 & 0 \\
\hline & $18-54 \mathrm{~h}$ after & 61 & 61 & 0 & 0 & 0 \\
\hline \multirow[t]{5}{*}{ RI ending } & 18-24 h before & 44 & 0 & 43 & 1 & 0 \\
\hline & $12-18 \mathrm{~h}$ before & 55 & 0 & 52 & 2 & 1 \\
\hline & $6-12 \mathrm{~h}$ before & 44 & 0 & 23 & 18 & 3 \\
\hline & 0-6 h before & 36 & 0 & 13 & 18 & 5 \\
\hline & tal & 595 & 192 & 306 & 86 & 11 \\
\hline
\end{tabular}

Table 3.3 shows the mean intensity, past 12-hr intensity change, SST, TPW, vertical wind shear magnitude, storm motion speed, and the degree of difference between shear and motion directions. The differences of each parameter between RI and SI are prominently small. It indicates that as long as a TC in environmental conditions that are more favorable than a certain level, it will be difficult to predict it is going to RI or SI solely based on the environmental information. Based on the statistical study (Hendricks et al. 2010), it is found that rapid intensification is mostly controlled by internal dynamical processes under favorable environmental conditions. 
Table 3.3: Mean values of maximum wind, Sea Surface Temperature (SST), Total Precipitable Water (TPW), vertical wind shear magnitude, storm motion speed, and the direction difference between motion and vertical wind shear in degree for different intensity change categories and total dataset.

\begin{tabular}{l|ccccc}
\hline \hline Mean Values & $\mathrm{W}$ & $\mathrm{N}$ & $\mathrm{SI}$ & $\mathrm{RI}$ & Total \\
\hline Vmax $(\mathrm{kt})$ & 63.1 & 53.5 & 53.3 & $61^{\mathrm{a}}$ & 56.2 \\
SST $\left({ }^{\circ} \mathrm{C}\right)$ & 27.5 & 27.9 & 28.4 & 28.7 & 28.1 \\
TPW $(\mathrm{mm})$ & 61.2 & 61.5 & 63.0 & 63.3 & 62.1 \\
Shear $\left(\mathrm{m} \mathrm{s}^{-1}\right)$ & 8.8 & 7.9 & 6.4 & $5.5^{\mathrm{a}}$ & 7.4 \\
Motion $\left(\mathrm{m} \mathrm{s}^{-1}\right)$ & 4.7 & 4.3 & 4.3 & 4.5 & 4.4 \\
Shear - Motion & & & & & \\
$\left(^{\circ}\right)$ & -69.2 & -59.0 & -57.1 & -62.2 & -60.8 \\
\hline
\end{tabular}

a The statistical significance of the value from the value in the SI column at the $99.9 \%$ confidence level.

The mean values for of the maximum sustained winds, SST, shear magnitude, TPW, storm motion, and the discrepancy between the direction of shear and motion vector are calculated in Tao (2016) with the same dataset. Storms that have been undergoing RI for over 12 hours (i.e., RI CON) present significantly higher intensity and lower vertical wind shear than those within 12 hours from the RI onset (i.e., RI INI). The SST decreases significantly 12 hours following the period of RI continuing, while the intensity and vertical wind shear significantly increase from 12-24 to 0-12 hours before RI ends.

\subsubsection{Definition of three kinds of precipitation}

TJ15 defined four kinds of precipitation depending on the height of 20-dBZ radar echo and shows different types of precipitation have different contributions to TC intensification. In this paper, similarly, we use TMI 2A12 rain rate to define four types of 
precipitation. As in Chapter 2, the TMI 2A12 rain rate $(\mathrm{R})$ is categorized into four precipitation types: total precipitation $\left(R \geq 0.5 \mathrm{~mm} \mathrm{hr}^{-1}\right)$, heavy precipitation $(\mathrm{R} \geq 10 \mathrm{~mm}$ $\mathrm{hr}^{-1}$ ), moderate precipitation (10 $\mathrm{mm} \mathrm{hr}^{-1}>\mathrm{R} \geq 5 \mathrm{~mm} \mathrm{hr}^{-1}$ ), and light precipitation (5 mm $\mathrm{hr}^{-1}>\mathrm{R} \geq 0.5 \mathrm{~mm} \mathrm{hr}^{-1}$ ). Since the TMI V7 oceanic algorithm gives a high number of false positive rain rates, we use a threshold rain rate of $0.5 \mathrm{~mm} \mathrm{~h}^{-1}$ with a probability $95 \%$ of rain as the minimum criterion of raining $(\mathrm{ZJ} 14)$

\subsubsection{Fourier Decomposition}

Two-dimensional (2D) composite images are used to show the results. Every TMI overpass has been rotated to make the TC environmental vertical wind shear point to the north before compositing. The overpasses of TC located in Southern Hemisphere are mirrored for the composite (Chen et al. 2006; Wingo and Cecil 2010; TJ15; Tao et al. 2017). Composite image of two different parameters will be used to represent the results. The two parameters are separately percentage occurrence and rainfall asymmetry quantified by Fourier decomposition.

Similar to Lonfat et al. (2004), Chen et al. (2006), and Yu et al. (2015), the Fourier decomposition is applied to quantify the rainfall asymmetry. 10-km-wide annuli around the TC center are used to compute the spatial asymmetry of different intensity of TMI 2A12 rain rate. In each annulus, the Fourier coefficients are computed using the following equation (Stull 1987):

$$
a_{n}=\frac{1}{N} \sum_{k=0}^{N-1} R(k) \cos \left(\frac{2 \pi n k}{N}\right)
$$




$$
b_{n}=-\frac{1}{N} \sum_{k=0}^{N-1} R(k) \sin \left(\frac{2 \pi n k}{N}\right)
$$

where $R(k)$ is each individual rain rate, $\mathrm{n}$ is the wavenumber, $\mathrm{N}$ equal to 360 (Lonfat et al. 2004) is the total number of points being analyzed in each annulus, and $\mathrm{k}$ is the index of each point.

In this research, we analyze the dominant asymmetries as shown in Chapter 2, which is the sum of the wavenumber 1 through 6 amplitudes divided by the mean rain rate over the entire annulus. The spatial structure of the dominant-order asymmetry $\left(R_{1-6} / R_{0}\right)$ can be represented by

$$
\frac{R_{1-6}}{R_{0}}=\frac{\sum_{D W} a_{n} \cos \left(\frac{2 \pi n k}{N}\right)-b_{n} \sin \left(\frac{2 \pi n k}{N}\right)}{R_{0}}
$$

$D W$ means "dominant wavenumbers" (for example, wavenumber n equals to 1 through 6), $R_{0}$ is the mean rain rate calculated over the entire annulus.

\subsection{Results}

\subsubsection{RI vs. non-RI}

Figure 3.1 shows the composite of the shear-relative $R_{1-6} / R_{0}$ for total precipitation. The composites of $R_{1-6} / R_{0}$ consistently show that downshear-left quadrant is favored for the maximum asymmetries for all four intensity change categories as the previous study of Chen et al. (2006) and Chapter 2 of this dissertation. In Fig. 3.1, weakening (W) storms shows the largest area of $R_{1-6} / R_{0}$ higher than $50 \%$. The areas of the $R_{1-6} / R_{0}$ higher than $50 \%$ for both $\mathrm{W}$ and neutral $(\mathrm{N})$ extend from near the TC center to the radius of $200 \mathrm{~km}$ in the 
downshear-left region. Slowly intensifying (SI) storms have a very small area of $R_{1-6} / R_{0}$ higher than $50 \%$. The maximum $R_{1-6} / R_{0}$ of SI storms are located downshear-left at the radius of $100 \mathrm{~km}$ to $200 \mathrm{~km}$. For RI storms, the composite shows small values of maximum $R_{1-6} / R_{0}$, which is in generally lower than those of SI storms. The maximum $R_{1-6} / R_{0}$ is located downshear-left at the radius of 100 to $200 \mathrm{~km}$. Table 3.4 shows the asymmetry index of the shear-relative composite $R_{1-6} / R_{0}$ of four kinds of precipitation and four different intensity change categories. The asymmetry index is defined as the maximum $R_{1-}$ ${ }_{6} / R_{0}$ to serve as an estimate of the degree of precipitation asymmetry. In Table 3.4 , for the total precipitation, the asymmetry index of the four categories ranks from the highest to the lowest as N, W, SI, and RI. Figure 1 and Table 3.4 collectively indicate that for the nonintensifying storms ( $\mathrm{W}$ and $\mathrm{N}$ ), there is a large area of asymmetries higher than $50 \%$ and a large asymmetry index. $R_{1-6} / R_{0}$ higher than $50 \%$ (high asymmetry) extends starting from near the TC center. For the intensifying storms (SI and RI), the area of $R_{1-6} / R_{0}$ higher than $50 \%$ is very small and the maximum $R_{1-6} / R_{0}$ tend to be located at an outer region at around $150 \mathrm{~km}$. The maxima of asymmetries for SI and RI are also smaller. Relative to shear direction, RI storms has the most symmetric wavenumber 1-6 perturbation; however, W storms have the most asymmetric wavenumber 1-6 perturbation. 
(a) W

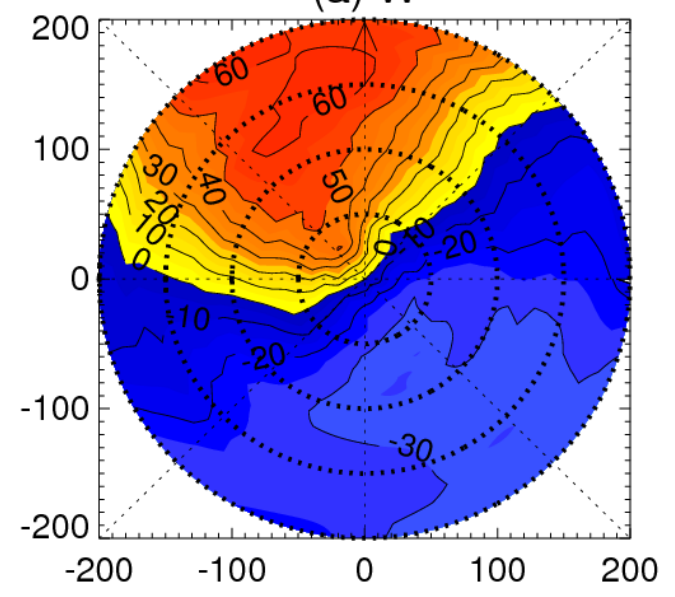

(c) $\mathrm{SI}$

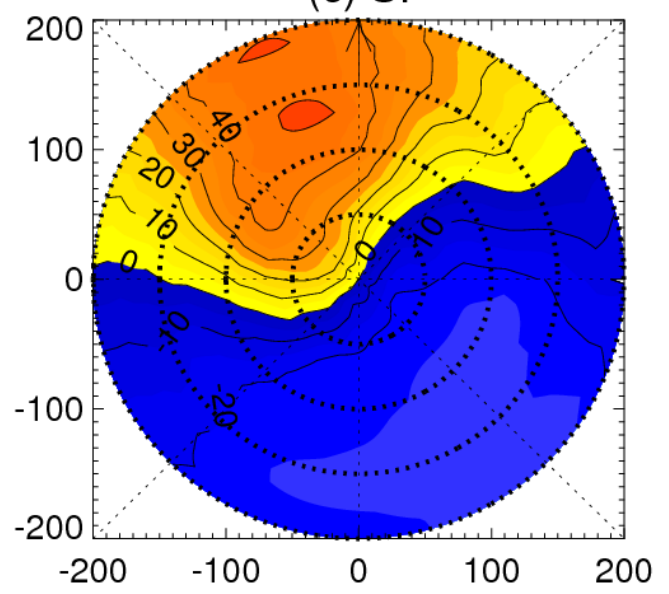

(b) $\mathrm{N}$

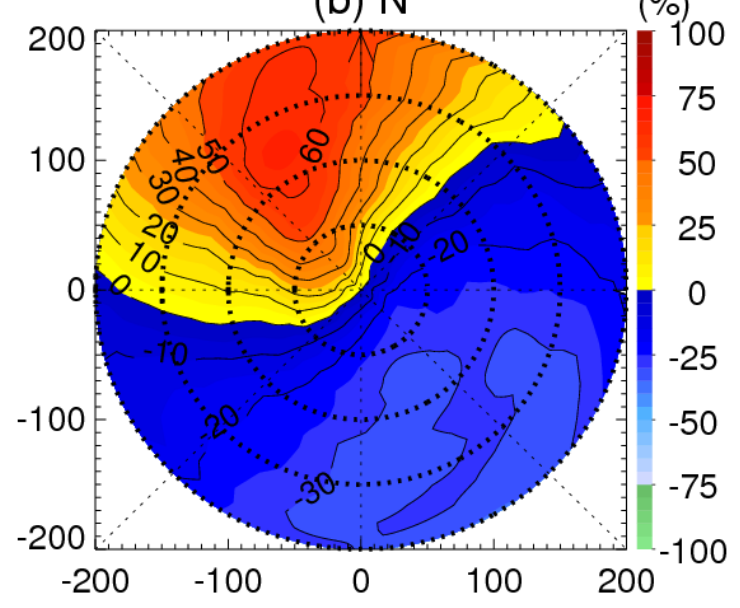

(d) RI

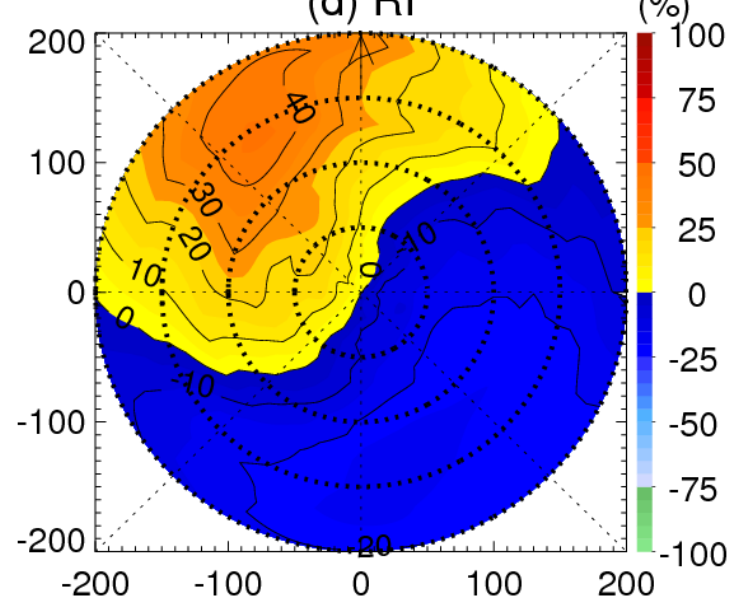

Figure 3.1: Composite shear-relative $\mathrm{R}_{1-6} / \mathrm{R}_{0}$ for total precipitation for (a) $\mathrm{W}$, (b) $\mathrm{N}$, (c) SI, (d) RI 
Table 3.4: The asymmetry index of the composite shear-relative $R_{1-6} / R_{0}$ of total precipitation, light precipitation, moderate precipitation, and heavy precipitation of [four columns] weakening, neutral, slowly intensifying, and rapid intensifying TCs.

\begin{tabular}{l|cccc}
\hline \hline$\%$ & $\mathrm{~W}$ & $\mathrm{~N}$ & $\mathrm{SI}$ & $\mathrm{RI}$ \\
\hline Total & 64.7 & 67.2 & 51.8 & 46.2 \\
Light & 44.6 & 38.5 & 30.8 & 24.7 \\
Moderate & 62.8 & 70.6 & 50.2 & 52.5 \\
Heavy & 67.0 & 73.2 & 68.1 & 61.4 \\
\hline
\end{tabular}

The shear-relative composite $R_{1-6} / R_{0}$ for light precipitation is shown in Fig. 3.2 for all four intensity change categories. It shows that for $\mathrm{W}$ storms, light precipitation is more symmetric in the inner core than the outer region. Unlike the composite $R_{1-6} / R_{0}$ of total precipitation for $\mathrm{W}$ storms (Fig. 3.1a) that the high $R_{1-6} / R_{0}$ (higher than $50 \%$ ) area extends from near the TC center to the outer region, the $R_{1-6} / R_{0}$ higher than $20 \%$ for light precipitation (Fig. 3.2a) of weakening storms is mainly located at downshear at from 100 to $200 \mathrm{~km}$ radius in the front of TC center. In the area within $100 \mathrm{~km}$ radius for weakening storms, the $R_{1-6} / R_{0}$ composite of light precipitation is lower than that in the outer region (100 to $200 \mathrm{~km}$ radius) of downshear. Downshear-left is favored by the highest precipitation in the inner core region for weakening storms, but in the outer region, downshear is more favored. For $\mathrm{N}$ storms, the maximum $R_{1-6} / R_{0}$ is located at downshearleft at the radius from 100 to $150 \mathrm{~km}$, but is located at downshear-right from 50- to 100 $\mathrm{km}$ radius. From 0 to $50 \mathrm{~km}$ radius, the maximum $R_{1-6} / R_{0}$ is located at downshear-left. For SI storms, the light precipitation asymmetries are located mainly downshear-left at all the radii. For RI storms, the maximum $R_{1-6} / R_{0}$ are at downshear-left at the radius from 150 to $200 \mathrm{~km}$. But in the inner core region (from radius 0 to $100 \mathrm{~km}$ ), the maximum light 
(a) W

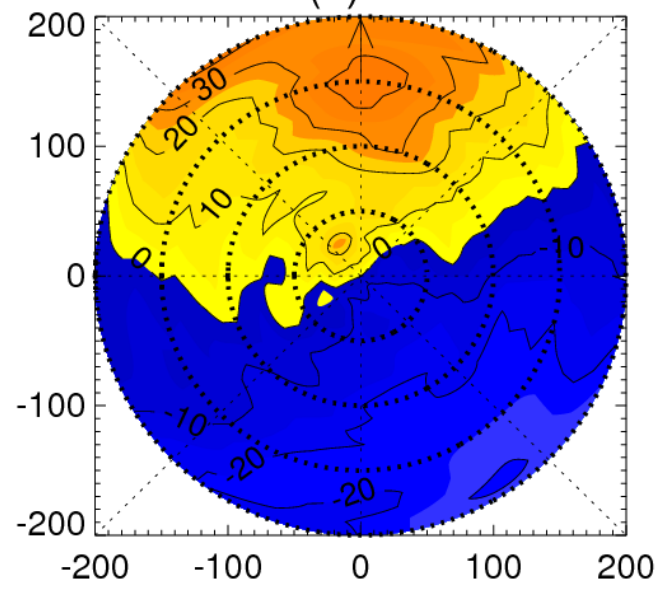

(c) $\mathrm{SI}$

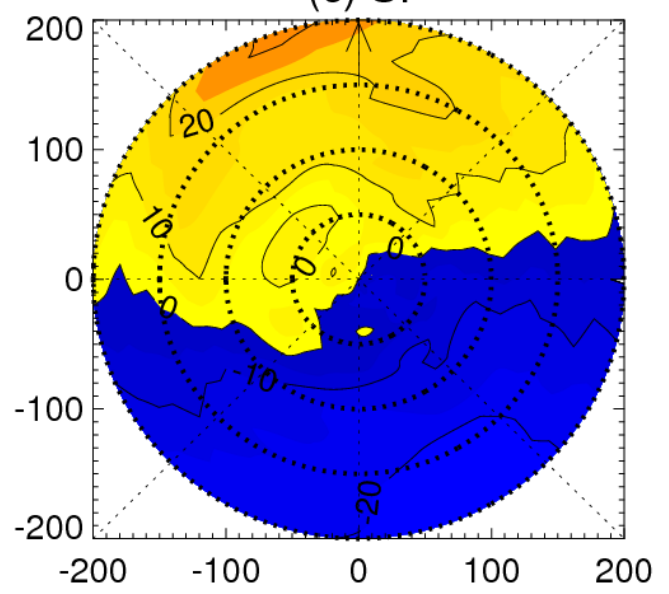

(b) $\mathrm{N}$

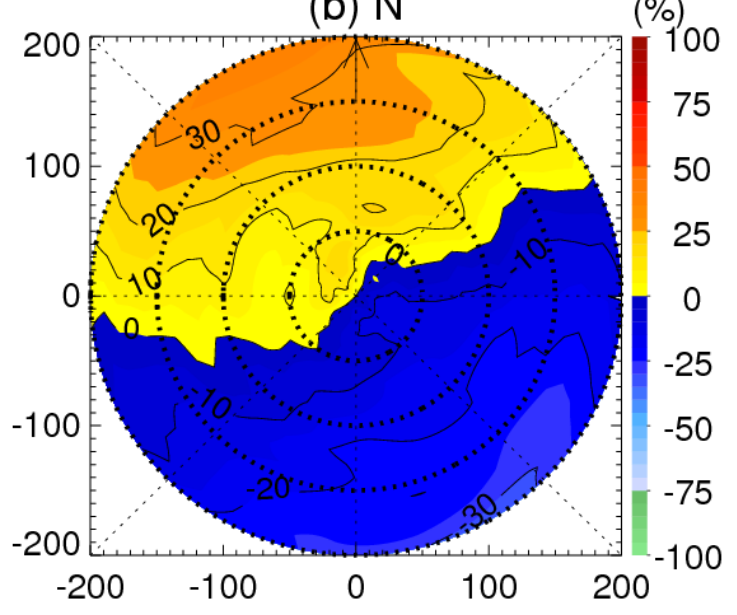

(d) RI

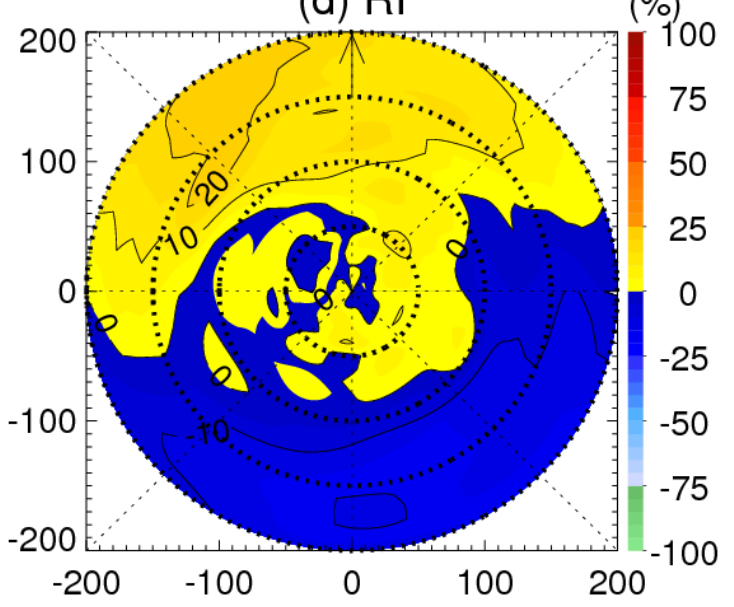

Figure 3.2: Composite shear-relative $\mathrm{R}_{1-6} / \mathrm{R}_{0}$ for light precipitation for (a) W, (b) $\mathrm{N}$, (c) SI, (d) RI

precipitation asymmetries are located at downshear-right. In the inner core region, the positive $R_{1-6} / R_{0}$ of light precipitation are spread out around the center, with components mostly at right of shear. In Table 3.4, the asymmetry index of light precipitation for the four categories ranks from the highest to the lowest as W, N, SI, and RI. However, the 
asymmetry indexes of light precipitation are averagely $20 \%$ lower than those of total precipitation, which indicates that light precipitation has small perturbation energy.

For moderate precipitation, in Fig. 3.3 it shows that downshear-left quadrant is also favored for the positive asymmetries for all four intensity change categories. The inner core region of weakening storms shows more symmetric moderate precipitation than in the outer region since from 0 to $150 \mathrm{~km}$ radius the maximum asymmetry is less than $50 \%$. For neutral storms, the area of asymmetry higher than $50 \%$ for moderate precipitation is located in the outer region. The $50 \%$ contour of $\mathrm{N}$ storms are larger and spread at more radii from 50 to $200 \mathrm{~km}$ than W storms. For both SI and RI storms, the overall asymmetries are smaller than those of $\mathrm{W}$ and $\mathrm{N}$ storms. The composite of SI storms shows a broader area of asymmetries higher than $40 \%$ than RI storm. But the moderate precipitation asymmetry composite of RI storms shows more symmetric in the inner core than those of SI, N, and W storms. It shows most of the asymmetry in the inner core region of RI storms is less than $10 \%$; however, for $\mathrm{W}, \mathrm{N}$, and $\mathrm{SI}$, the composites show the asymmetry in the inner core can be as high as 30\%. In Table 3.4, the asymmetry index of moderate precipitation for the four categories ranks from the highest to the lowest as N, W, RI, and SI. The asymmetry index of $\mathrm{RI}$ is higher than $\mathrm{SI}$ is because, in the outer region of RI composite, there is a very small area with asymmetries higher than 50\%. But the area of asymmetries higher than $30 \%$ of SI is larger than that of RI composite. 
(a) W

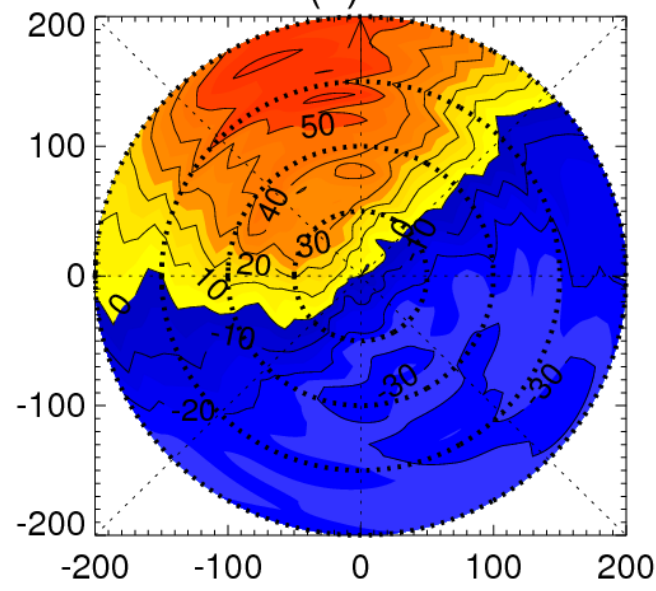

(c) $\mathrm{SI}$

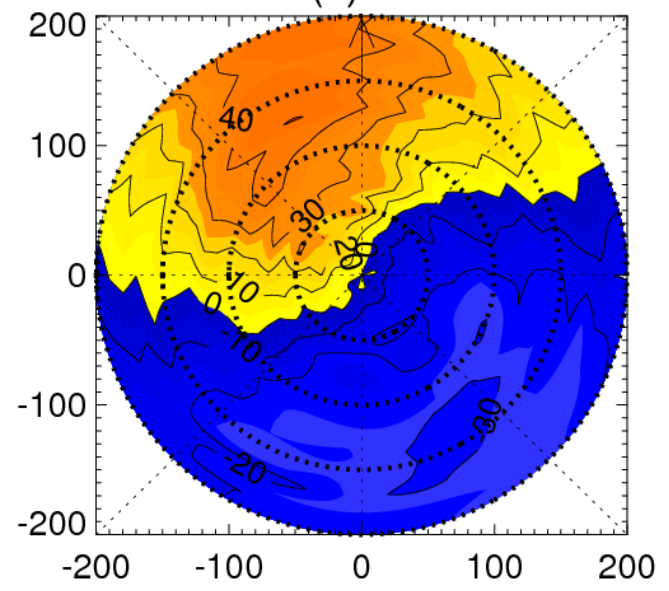

(b) $\mathrm{N}$

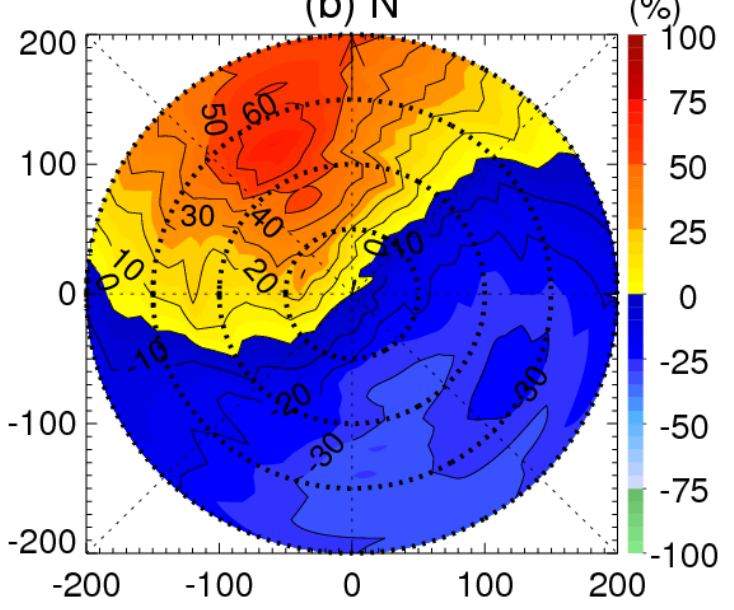

(d) RI

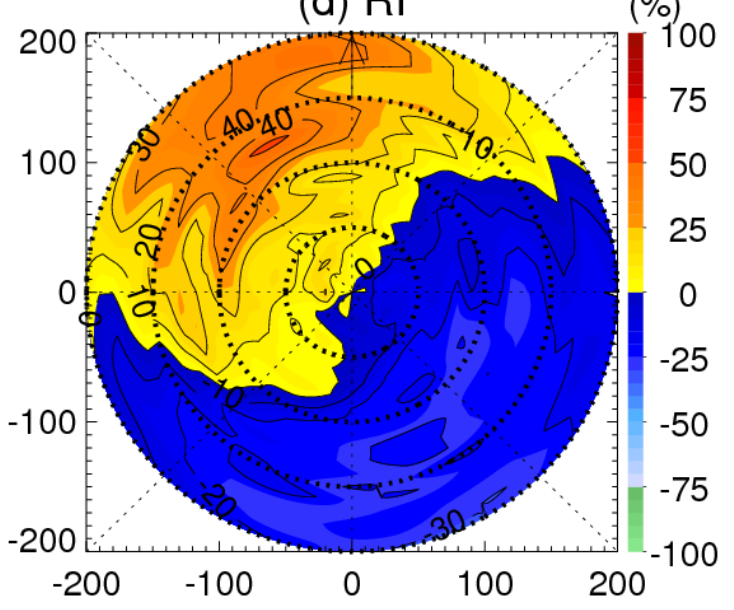

Figure 3.3: Composite shear-relative $\mathrm{R}_{1-6} / \mathrm{R}_{0}$ for moderate precipitation for (a) W, (b) $N$, (c) SI, (d) RI

Figure 3.4 shows for all four intensity change categories, the positive heavy precipitation asymmetries of each composite are located at downshear-left region. The area of asymmetries higher than $50 \%$ is the largest for $\mathrm{N}$ category, which is higher than $\mathrm{W}$ category. The area of $50 \%$ contour of SI is smaller than $\mathrm{W}$ and $\mathrm{N}$ composite and larger than RI composite. The asymmetry composite of RI has the smallest area of asymmetries higher 
than 50\%. As shown in Table 3.4, the asymmetry index of heavy precipitation ranks from the highest to the lowest for all the categories as N, SI, W, and RI. The asymmetry composite of RI is the most symmetric. Though the SI storm composite has a higher asymmetry index than $\mathrm{W}$ storm, the area of asymmetries higher than $50 \%$ of SI storm is smaller than that of W storm. Overall comparing the asymmetry in the inner core region for all different four kinds of precipitation for RI storms, the asymmetry of heavy precipitation is higher than that of the other three types of precipitation in each intensity change category, respectively. The radius of maximum wind (RMW) values were shown as $43 \mathrm{~km}$ for intensifying and 53km for steady-state TCs in Rogers et al. (2013). Though RI storms show symmetric precipitation properties for total, light, and moderate precipitation (Fig. 3.1d, 3.2d, and 3.3d), asymmetric heavy precipitation near or within the RMW has been linked to vortex (e.g., Vigh and Schubert 2009; Pendergrass and Willoughby 2009; Sitkowski and Barnes 2009; Reasor et al. 2009; Molinari and Vollaro 2010; Rogers 2010; Nguyen and Molinari 2012; Rogers et al. 2013).

As in TJ15, the shallow and moderate precipitation (defined as TRMM precipitation radar $20 \mathrm{dBZ}$ height lower than $10 \mathrm{~km}$ in their paper) increased at the onset stage of RI in the upshear quadrants and become more symmetric in the RI continuing stages. The increase of shallow and precipitation in upshear quadrant will enhance the symmetry of precipitation. Our results indicate the four types precipitation defined in this paper, especially the light precipitation, are all more symmetric for RI storms, which is consistent with TJ15. 
(a) W

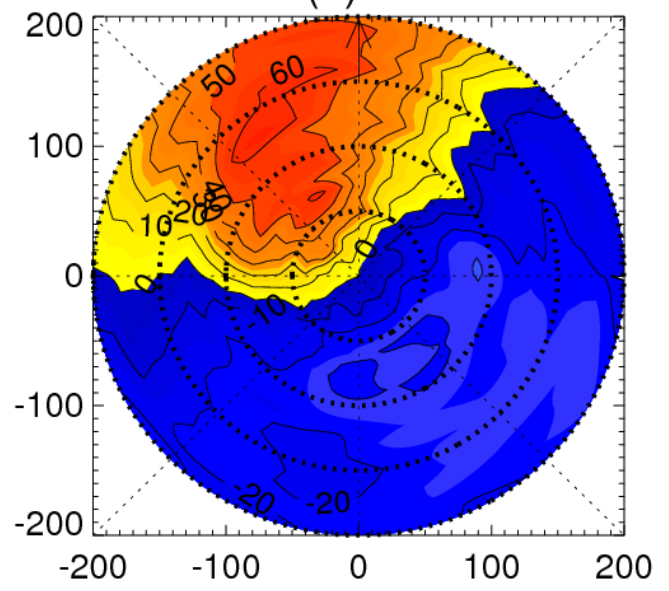

(c) $\mathrm{SI}$

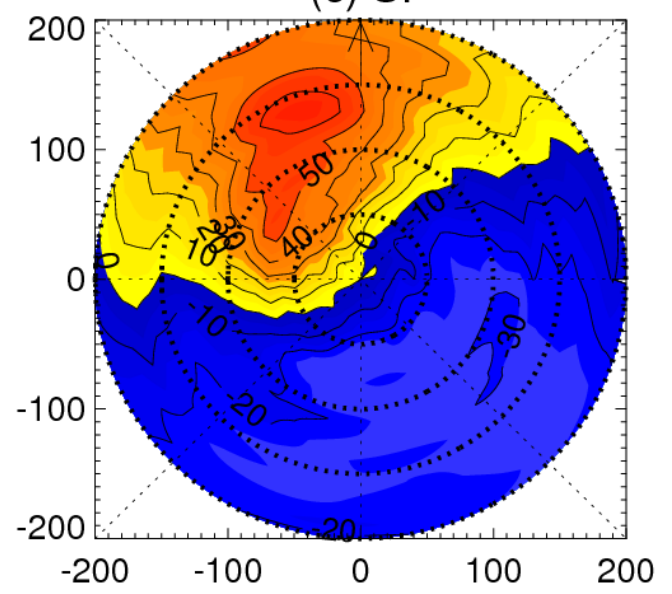

(b) $\mathrm{N}$

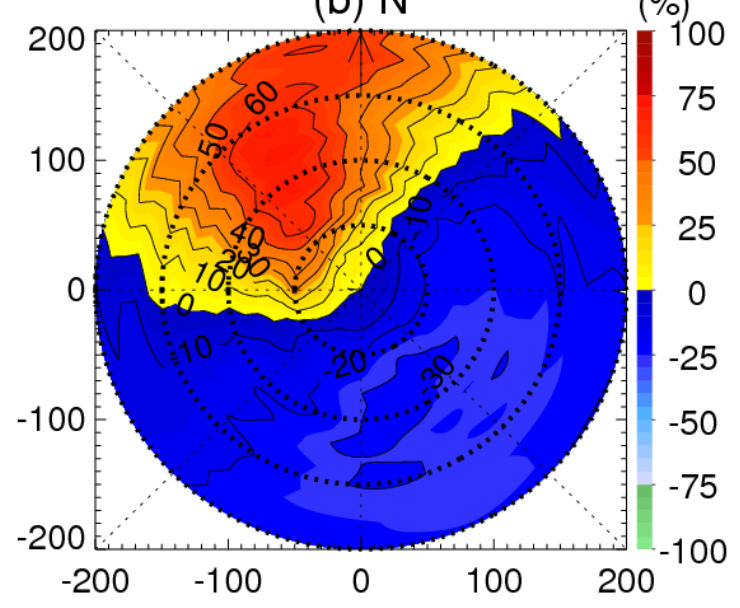

(d) RI

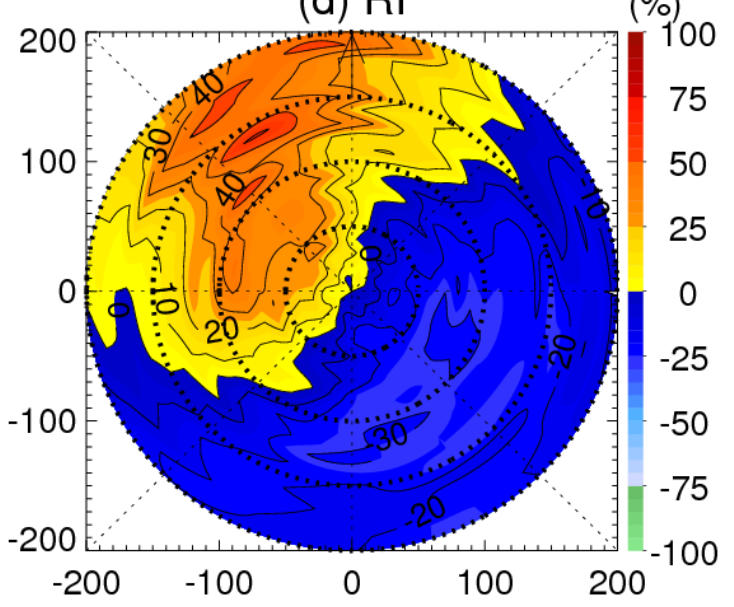

Figure 3.4: Composite shear-relative $\mathrm{R}_{1-6} / \mathrm{R}_{0}$ for heavy precipitation for (a) W, (b) $\mathrm{N}$, (c) SI, (d) RI

\subsubsection{RI event}

The shear-relative composites of $R_{1-6} / R_{0}$ of total precipitation in Fig. 3.5I shows a downshear-left dominant distribution for all RI event-based categories, consistent with previous studies (e.g., Chen et al. 2006; Wingo and Cecil 2010; ZJ14; TJ15; Tao et al. 
2017). The positive asymmetry perturbation in the inner core region of TCs keeps decreasing until the beginning of RI ending period (Fig. 3.5Ie), and the decrease is most significant from $12 \mathrm{~h}$ before the onset of RI to RI initial stage.
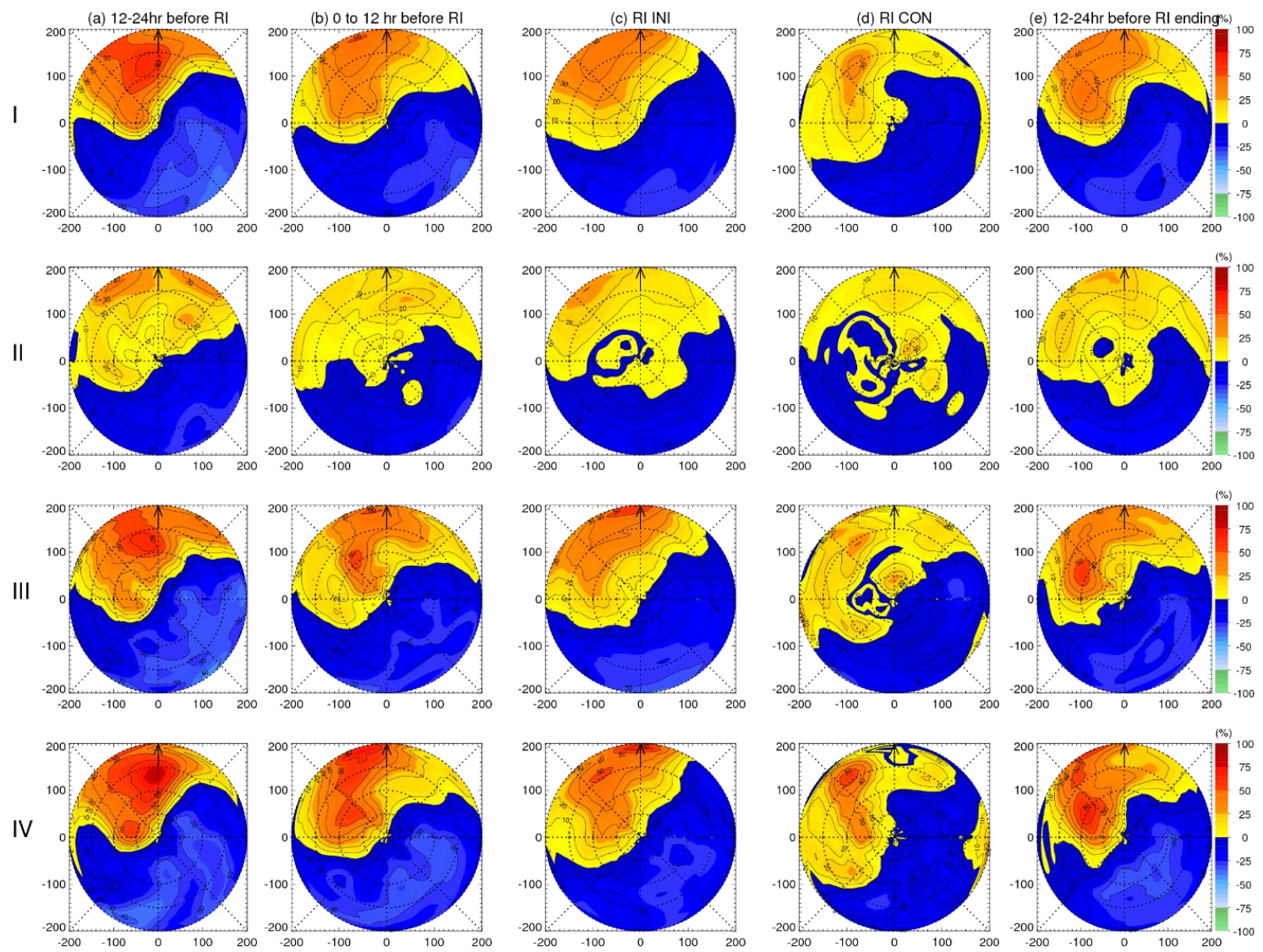

Figure 3.5: Composite shear-relative $\mathrm{R}_{1-6} / \mathrm{R}_{0}$ for (I) total precipitation, (II) light precipitation, (III) moderate precipitation, and (IV) heavy precipitation. (a) $12-24 \mathrm{~h}$ before RI onset, (b) 0-12 h before RI onset, (c) RI initial, (d) RI continuing, and (e) 12-24 h before RI ends. Dotted range rings represent the 25-, 50-, 75-, and 100-km radii.

The difference between total precipitation $R_{1-6} / R_{0}$ composites of $12-24 \mathrm{hr}$ before RI (Fig. 3.5Ia) and 0 to $12 \mathrm{hr}$ before RI (Fig. 3.5Ib) shows the decreases of the positive $R_{1-6} / R_{0}$ is mostly from the two downshear quadrants. In the meantime, the absolute value of negative $R_{1-6} / R_{0}$ perturbation also gets decreased. The decrease of the absolute value of 
negative $R_{1-6} / R_{0}$ represents an increase of precipitation at upshear. It signifies the importance of increasing the azimuthal rainfall symmetry around the TC center (Kieper and Jiang 2012; ZJ14; TJ15; Alvey et al. 2015). Alvey et al. 2015 demonstrated a similar trend of increasing precipitation symmetry from before RI to after RI. After the storm intensified, Fig. 3.5Id shows that the precipitation field is the most symmetric in the RI continuing stage. In Table 3.5, it clearly shows that for total precipitation, the asymmetry index decreases from $12-24 \mathrm{hr}$ before RI to RI continuing (RI CON) stages and starts to increase at 12-24 hours before RI ends. To examine how different precipitation types evolve, Figs. 3.5II, 3.5III, and 3.5IV shows the shear-relative composite image of the $R_{1 \text { - }}$ ${ }_{6} / R_{0}$ of light, moderate, and heavy respectively.

As shown in Fig. 3.5II, the composite $R_{1-6} / R_{0}$ of light precipitation at each stage of an RI event shows a more symmetric pattern. The asymmetry indexes in each stage of an RI event for light precipitation in Table 3.5 are smaller than those for total, moderate, and heavy precipitation. The positive asymmetries for light precipitation show different locations than that for total precipitation relative to vertical wind shear. The inner region of light precipitation in an RI life cycle is more symmetric than the outer region (100-200 $\mathrm{km})$. Fig. 3.5IIa shows that $12-24 \mathrm{hr}$ before RI the light precipitation positive $R_{1-6} / R_{0}$ are distributed downshear. As it can be seen that three areas of $R_{1-6} / R_{0}$ higher than $30 \%$ are located at downshear left from the radius of 150 to $200 \mathrm{~km}$, downshear right from the radius of 150 to $200 \mathrm{~km}$, and downshear right from the radius of 100 to $150 \mathrm{~km}$. The asymmetry index of TC light precipitation of $12-24 \mathrm{hr}$ before RI is $46 \%$ higher than that of $0-12 \mathrm{hr}$ before RI (Table 3.5). In Fig. 3.5IIb, it shows that the maximum asymmetry of the composite of 0 to $12 \mathrm{hr}$ before RI is located at downshear right at the radius from 100 to 
$200 \mathrm{~km}$. As an RI storm proceeds to the RI initial (RI INI) stage, the light precipitation gets more asymmetric. The light precipitation asymmetry index of RI INI is $9.4 \%$ higher than that of $0-12 \mathrm{hr}$ before RI. The maximum asymmetry of RI INI storms is located at downshear left at the outer region (from 150 to $200 \mathrm{~km}$ radius). In the inner core region of RI INI storms, it shows more symmetric pattern than the composite of 0 to $12 \mathrm{hr}$ before RI. The light precipitation asymmetry index of RI CON storms is 3\% higher than that of RI INI storms. The maximum asymmetry of RI CON storms is located at downshear at the outer region (from 150 to $200 \mathrm{~km}$ radius) and downshear right in the inner core region. The positive asymmetries are distributed around the TC center in the inner core region of RI CON storms. At the stage of 12-24 hr before RI ending, the light precipitation becomes more symmetric than the previous stage (RI CON stage). The asymmetry index of RI CON storms is $7 \%$ higher than that of $12-24 \mathrm{hr}$ before RI ending. Along the life cycle of an RI event, light precipitation shows the smallest value of asymmetry index at the stage of 0-12 hr before RI. Before a TC rapidly intensifies, symmetric light precipitation has a predictive power of TC RI.

For moderate precipitation, the maximum asymmetries are located at downshear left (Fig. 3.5IIIa,b,d) for storms at 12-24 hr before RI, 0 to $12 \mathrm{hr}$ before RI, and 12-24 hr before RI ending, which is similar to Fig. 3.5 Ia,b,d. However, for both RI INI and RI CON stages, the composites of moderate precipitation show different patterns than those of total precipitation. The maximum asymmetry of moderate precipitation is located in downshear left in the inner core region and downshear in the outer region for RI INI storms. For RI $\mathrm{CON}$ storms, the maximum asymmetry is located in downshear left in the outer region but downshear in the inner core region. In Table 3.5, it shows that the asymmetry index 
decreases in each stage from 12-24 hr before RI to RI CON stage. It increases from the stage of RI CON to $12-24 \mathrm{hr}$ before RI ending.

For heavy precipitation, the locations of maximum asymmetries resemble that of moderate precipitation for all the stages relative to RI except for RI CON storms. For RI $\mathrm{CON}$, the location of maximum asymmetries is clearly at downshear left, similar as that of RI CON for total precipitation. Similar to total and moderate precipitation, in Table 3.5, the asymmetry index of heavy precipitation decreases in each stage from 12-24 hr before RI to RI CON stage and increases from the stage of RI CON to 12-24 hr before RI ending. Except for light precipitation, total, moderate, and heavy precipitation decreases its asymmetry as a storm approaching to rapidly intensify and increases its asymmetry before a storm stops to rapidly intensify. However, light precipitation shows its predictive power for RI CON and RI ending with lower asymmetry index.

\subsubsection{Time evolution}

The time evolution of azimuthally averaged asymmetry index for 0-200 km radii before and throughout the RI events are examined in Fig. 3.6. The asymmetry index for each radius is defined as the maximum $R_{1-6} / R_{0}$ of each radius. The Hovmöller diagrams shown in Fig. 3.6 are generated by grouping the asymmetries from 14 RI event-based categories (Table 3.2) into $10-\mathrm{km}$ annuli bins extending radially outward from the center. Generally, the evolution of asymmetry index changes little with time from 0 to $10 \mathrm{~km}$ radius for all four types of precipitation.

The evolution of asymmetry index indicates a large asymmetric total precipitation from 45 to $21 \mathrm{hr}$ before RI. From 45 to $21 \mathrm{hr}$ before RI, the asymmetry index is generally 
increasing. By the time of $21 \mathrm{hr}$ before RI, at the radius of $100 \mathrm{~km}$, it shows the largest asymmetry index along the timeline among all the radii. After the time point of $-21 \mathrm{hr}$, a clear signal of asymmetry index decreasing is shown. Between 21 and $9 \mathrm{hr}$ before RI, the asymmetry index becomes smaller at all the radii. 3 to $9 \mathrm{hr}$ before RI, in the inner core region (0-100 km radius), the asymmetry indexes are getting smaller. $3 \mathrm{hr}$ before RI, the asymmetry index of both the inner and outer region also decreases. The signal of asymmetry index decreasing indicates its predictive power. But from 3 to $0 \mathrm{hr}$ before RI, the asymmetry index of the storm outer region is higher than that in the inner core. From 0 to $24 \mathrm{hr}$ during RI, it is the RI INI stage. From 0 to $9 \mathrm{hr}$ after RI, the asymmetry index increases at the radius from 50 to $200 \mathrm{~km}$. After the time line of $24 \mathrm{hr}$ after RI, it shows small asymmetry index throughout all the radii, which corresponds to the RI continuing stage. Starting from $0 \mathrm{hr}$ before RI ends, the asymmetry index started to increase. It increases more in the region of radius from 50 to $100 \mathrm{~km}$ than in the inner region of 0 to $50 \mathrm{~km}$ radius. $3 \mathrm{hr}$ before $\mathrm{RI}$ ending, the asymmetry index increases a large amount at the radius of $150 \mathrm{~km}$.

The asymmetry indexes of light precipitation are smaller than those of total precipitation throughout the life cycle of an RI event as shown in Fig. 3.5b. From 45 to 15 hr before RI, the asymmetry indexes of light precipitation show lower asymmetry in the inner region from 0 to $50 \mathrm{~km}$ radius, shows higher asymmetry in the outer region of 50 to $200 \mathrm{~km}$ radius. There is a clear signal of symmetrization at the time line from 15 to $0 \mathrm{hr}$ before RI. It shows that especially in the inner region of 0 to $50 \mathrm{~km}$ radius, the asymmetry index of light precipitation decreases dramatically. Right after the light precipitation symmetrization period, it is the RI stage. At the beginning 0 to $9 \mathrm{hr}$ of RI, the outer region 
of the storm shows larger asymmetry indexes. But after the storm continues rapidly intensifying for $24 \mathrm{hr}$, the asymmetry index of all the radii becomes lower than $20 \%$, which is the most symmetric stage. Starting from $24 \mathrm{hr}$ before RI ending, the light precipitation becomes more asymmetric as time proceeding.

For moderate precipitation, its asymmetry indexes start increasing from 45 to $21 \mathrm{hr}$ before RI at all the radii. Between 21 to $15 \mathrm{hr}$ before RI, there is a small decrease in asymmetry index. But after that, the asymmetry index continues to increase and then decreases before RI starts. During the RI period, the asymmetry index first becomes smaller in the inner core region at 0 to $24 \mathrm{hr}$ after RI starts. When RI last more than $24 \mathrm{hr}$, the asymmetry indexes decrease at all the radii. From $24 \mathrm{hr}$ before RI ends, the asymmetry indexes start to increase in the outer region, but not increase much in the inner core region. The asymmetry index pattern evolution of moderate precipitation and heavy precipitation are very alike. Though, in general, heavy precipitation has higher asymmetry index than total, light, and moderate precipitation. Overall, Fig. 7 suggests that the onset of RI is associated with a significant symmetric precipitation pattern in the inner core. Right before RI, the symmetrization of for light precipitation shows predictive power of storm RI. 
(a) Total Precip.

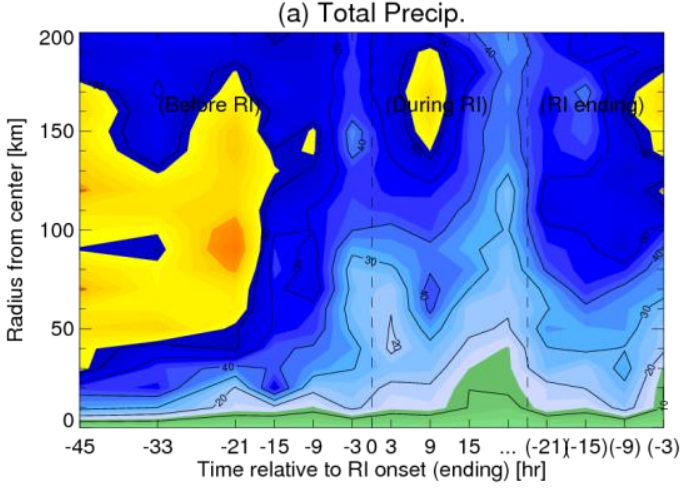

(c) Moderate Precip.

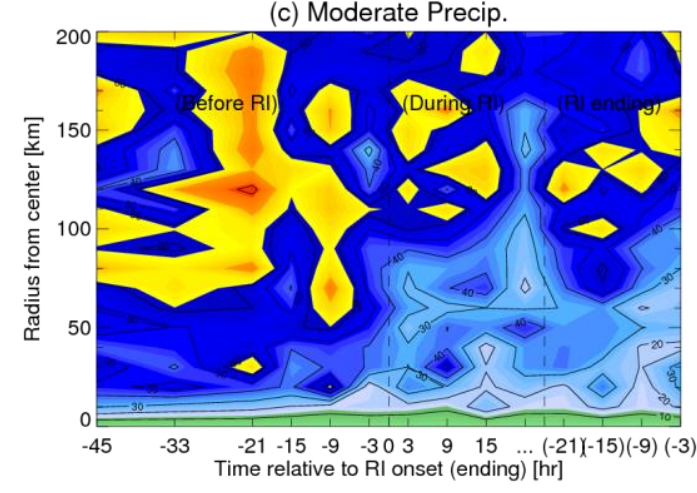

(b) Light Precip.

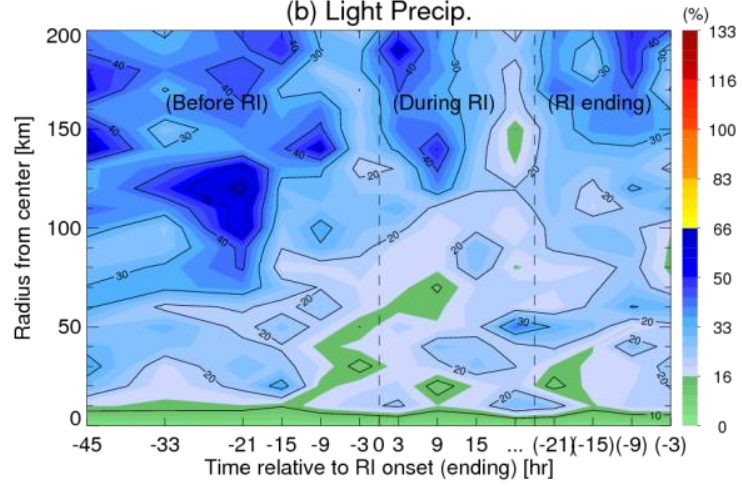

(d) Heavy Precip.

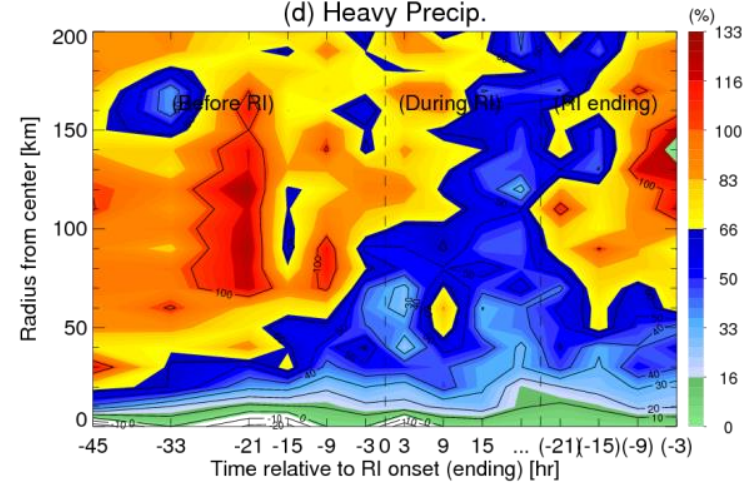

Figure 3.6: Hovmöller diagrams of azimuthal wavenumber-based Asymmetry Index from 0 to $200 \mathrm{~km}$ for (a) total precipitation, (b) light precipitation, (c) moderate precipitation, and (d) heavy precipitation. Dashed lines from left to right represent RI onset and $24 \mathrm{~h}$ before RI ends.

\subsection{Conclusions}

On the basis of 16 years of observations from TRMM TMI, the precipitation asymmetry properties of RI and non-RI storms are examined. Consistent with previous satellite-based studies on difference between RI and non-RI TCs (e.g., Jiang and Ramirez 2013; ZJ14, TJ15), the current paper in terms of the normalized (by azimuthal mean rainrate) wavenumber 1-6 asymmetry, indicates that in favorable environmental conditions, the lower asymmetry (wavenumber 1-6 perturbation normalized by azimuthal mean rain rate) of light precipitation are most strongly correlated with future intensity change. However, the asymmetry of heavy precipitation is higher than the other three types 
of precipitation in RI storms, which is a necessary condition for storm to intensify (Vigh and Schubert 2009; Pendergrass and Willoughby 2009; Sitkowski and Barnes 2009; Reasor et al. 2009; Molinari and Vollaro 2010; Rogers 2010; Nguyen and Molinari 2012; Rogers et al. 2013).

The distribution of the highest precipitation is also demonstrated to differentiate between different intensity change categories for four types of precipitation using the normalized wavenumber 1-6 asymmetry. It indicates that for total, moderate, and heavy precipitation, downshear-left quadrant is generally favored for the maximum asymmetries for all four intensity change categories (Chen et al. 2006). The light precipitation shows a different pattern of the normalized wavenumber 1-6 perturbation. As the maximum asymmetry of $\mathrm{W}$ storms is downshear. For both $\mathrm{N}$ and $\mathrm{SI}$ storms, the maximum asymmetry is located in dowshear left. The maximum asymmetry of RI storms is downshear in the outer region and very symmetric in the inner core region, which helps to predict RI. The corresponding asymmetry index is also defined quantitatively to demonstrate that RI storms are more symmetric in total, light, and heavy precipitation than the non-RI storms.

RI as an event, the evolution of asymmetry is also demonstrated. The results here document the evolution of different types of precipitation for RI storms. One of the most significant findings is the importance of the decrease of normalized wavenumber 1-6 asymmetries of all four types of precipitation several hours before RI onset. The decreases of asymmetries are mostly observed in the inner core region. This process of symmetrization is critical for storm RI, which is consistent with the previous studies (Alvey et al. 2015; Tao et al. 2017). Consistent with ZJ14, storms during RI (both RI INI and RI $\mathrm{CON}$ ) feature symmetric total precipitation in the inner region of radius less than $100 \mathrm{~km}$ 
and a more symmetric shear-relative azimuthal wavenumber 1-6 perturbation than those before RI onset and in the RI ending period. 


\section{REFERENCES}

Alvey III, G. R., J. Zawislak, and E. Zipser, 2015: Precipitation Properties Observed during Tropical Cyclone Intensity Change. Mon. Wea. Rev., 143, 4476-4492.

Burpee, R. W., M. L. Black, 1989: Temporal and Spatial Variations of Rainfall Near the Centers of Two Tropical Cyclones, Mon. Wea. Rev., 117(10), 2204-2218, doi: 10.1175/1520-0493(1989)117<2204:tasvor>2.0.co;2.

Cecil, D. J., 2007: Satellite-derived rain rates in vertically sheared tropical cyclones. Geophys. Res. Lett., 34, L02811, doi:10.1029/ 2006GL027942.

Chen, S. S., J. A. Knaff, and F. D. Marks, 2006: Effects of Vertical Wind Shear and Storm Motion on Tropical Cyclone Rainfall Asymmetries Deduced from TRMM, Mon. Wea. Rev., 134(11), 3190-3208, doi:10.1175/MWR3245.1.

Camargo, J. S., K. Emanuel, and A. Sobel, 2007: Use of a Genesis Potential Index to Diagnose ENSO Effects on Tropical Cyclone Genesis, 4819-4834, doi: 10.1175/JCLI4282.1.

M. Wheeler, and A. Sobel, 2009: Diagnosis of the MJO Modulation of Tropical Cyclogenesis Using an Empirical Index, 3061-3074.

https://doi.org/10.1175/2009JAS3101.1

Cangialosi, J. P., and J. L. Franklin, 2012: 2011 National Hurricane Center verification report. Tropical Prediction Center, National Hurricane Center, National Center for Environmental Prediction, National Weather Center, NOAA, 76 pp.

Corbosiero, K. L., and J. Molinari, 2002: The Effects of Vertical Wind Shear on the Distribution of Convection in Tropical Cyclones, Mon. Wea. Rev.,130(8), 2110-2123, doi: 10.1175/1520-0493(2002)130<2110:TEOVWS>2.0.CO;2.

— , and J. Molinari, 2003: The Relationship between Storm Motion, Vertical Wind Shear, and Convective Asymmetries in Tropical Cyclone, J. Atmos. Sci., 60(2), 366-376, doi:10.1175/1520-0469(2003)0602.0.CO;2.

DeMaria, M., M. Mainelli, L. K. Shay, J.A. Knaff, and J. Kaplan, 2005: Further Improvements to the Statistical Hurricane Intensity Prediction Scheme (SHIPS). Wea. Forecasting, 20, 531-543.

Elsberry, R. L., 2002: Predicting hurricane landfall precipitation: Optimistic and pessimistic views from the symposium on precipitation extremes. Bull. Amer. Meteor. Soc., 83(9), 1333-1339.

Frank, W. M.,1977: The Structure and Energetics of the Tropical Cyclone I. Storm Structure. Mon. Wea. Rev. doi: 10.1175/1520-0493(1977)105<1119:TSAEOT>2.0.CO;2. 
Gray, W. M., \& Brody, L. R., 1967: Global view of the origin of tropical disturbances and storms. Colorado State University, Department of Atmospheric Science.

— and Dynamic Characteristics. J. Atmos. Sci., 30.8 (1973): 1565-1576.. doi: 10.1175/15200469(1973)030<1565:THICRI $>2.0$. CO;2.

Hence, D. A., and Houze, R. A., 2011: Vertical Structure of Hurricane Eyewalls as Seen by the TRMM Precipitation Radar. J. Atmos. Sci., 68(8), 1637-1652. doi:

10.1175/2011JAS3578.1.

— , and Houze, R. A., 2012: Vertical Structure of Tropical Cyclone Rainbands as Seen by the TRMM Precipitation Radar. J. Atmos. Sci., 69(9), 2644-2661. doi: 10.1175/JASD-11-0323.1.

Hendricks, E. A., M. S. Peng, B. Fu, and T. Li, 2010: Quantifying Environmental Control on Tropical Cyclone Intensity Change. Mon. Wea. Rev., 138,

Houze, R. A., 2010: Clouds in Tropical Cyclones, Mon. Wea. Rev., 138(2), 293-344, doi: 10.1175/2009MWR2989.1.

Jiang, H., 2012: The relationship between tropical cyclone rapid intensification and the strength of inner-core convection. Mon. Wea. Rev., 140, 1164-1176.

— C. Ciu, and E. J. Zipser, 2011: A TRMM-based tropical cyclone cloud and precipitation feature database. J. Appl. Meteor. Climatol. 50,1255-1274.

—, Ramirez, E. M., \& Cecil, D. J. (2012). Convective and Rainfall Properties of Tropical Cyclone Inner Cores and Rainbands from 11 Years of TRMM Data. Mon. Wea. Rev., 120807114754001. doi: 10.1175/MWR-D-11-00360.1.

Johnny C. L. Chan, K. S. Liu, Simon E. Ching, and Edwin S. T. Lai, 2004: Asymmetric Distribution of Convection Associated with Tropical Cyclones Making Landfall along the South China Coast. Mon. Wea. Rev., 132, 2410-2420.

Kelley, O. A., J. Stout, and J. B. Halverson, 2004: Tall precipitation cells in tropical cycloneeyewalls are associated with tropical cyclone intensification. Geophys. Res. Lett., 31, L24112, doi: 10.1029/2004GL021616.

Kepert, J., 2001: The Dynamics of Boundary Layer Jets within the Tropical Cyclone Core. Part I: Linear Theory, J. Atmos. Sci., 58(17), 2469-2484, doi: 10.1175/15200469(2001)058<2469:TDOBLJ>2.0.CO\%3B2.

and Y. Wang, 2001: The Dynamics of Boundary Layer Jets within the Tropical Cyclone Core. Part II: Nonlinear Enhancement, J. Atmos. Sci., 58(17), 2485-2501, doi: $10.1175 / 1520-0469(2001) 058<2485 \% 3$ ATDOBLJ $>2.0 . C O \% 3 B 2$. 
Kieper, M. E., and H. Jiang, 2012: Predicting tropical cyclone rapid intensification using the $37 \mathrm{GHz}$ ring pattern identified from passive microwave measurements. Geophys. Res. Lett., 39, L13804, doi: 10.1029/2012GL052115.

Klotz, B.W. and H. Jiang, 2017: Examination of Surface Wind Asymmetries in Tropical Cyclones: Part I. General Structure and Wind Shear Impacts. Mon. Wea. Rev., doi: 10.1175/MWR-D-17-0019.1.

Kummerow, C., W. S. Olson, and L. Giglio, 1996: A simplified scheme for obtaining precipitation and vertical hydrometeor profiles from passive microwave sensors. IEEE Transactions on Geosci. and Remote Sensing. 34, 1213-32.

— , Y. Hong, W. S. Olson, S. Yang, R. F. Adler, J. McCollum, ... T. T. Wilheit, 2001: The Evolution of the Goddard Profiling Algorithm (GPROF) for Rainfall Estimation from Passive Microwave Sensors. J. Appl. Meteor., 40(11), 1801-1820. doi: 10.1175/1520-0450(2001)040<1801:TEOTGP>2.0.CO;2.

— - W. Barnes, T. Kozu, J. Shiue, and J. Simpson, 1998: The Tropical Rainfall Measuring Mission (TRMM) sensor package. J. Atmos. Oceanic Technol., 15(3), 809817. doi: 10.1175/1520-0426(1998)015<0809:TTRMMT>2.0.CO;2.

Lonfat, M., F. D. Marks, and S. S. Chen, 2004: Precipitation distribution in tropical cyclones using the tropical rainfall measuring mission (TRMM) microwave imager: A global perspective. Mon. Wea. Rev., 132, 1645-1660.

— R. Rogers, T. Marchok, and F. D. Marks, 2007: A Parametric Model for Predicting Hurricane Rainfall. Mon. Wea. Rev., 135(9), 3086-3097. https://doi.org/10.1175/MWR3433.1.

Marks, F. D., 1985: Evolution of the structure of precipitation in Hurricane Allen (1980). Mon. Wea. Rev., 113(6), 909-930, doi: 10.1175/15200493(1985)113<0909:EOTSOP>2.0.CO;2.

_ , and R. A. Houze, 1987: Inner Core Structure of Hurricane Alicia from Airborne Doppler Radar Observations, J. Atmos. Sci., 44(9), 1296-1317, doi: 10.1175/15200469(1987)044<1296:ICSOHA>2.0.CO;2.

— , and L. K. Shay, 1998: Landfalling tropical cyclones: Forecast problems and associated research opportunities. Bull. Amer. Meteor. Soc., 79, 305-323.

— G. Kappler, and M. DeMaria, 2002: Development of a tropical cyclone rainfall climatology and persistence (R-CLIPER) model. Preprints, 25th Conf. on Hurricanes and Tropical Meteorology, San Diego, CA, Amer. Meteor. Soc., 327-328.

McBride, J. L., 1979: Observational analysis of tropical cyclone formation. Atmospheric science paper; no. 308. 
Miller, B. I., 1958: On the Maximum Intensity of Hurricane, J. Meteorol., 15(2), 184195, doi: 10.1175/1520-0469(1958)015<0184:OTMIOH>2.0.CO\%3B2.

Montgomery, M. T., M. E. Nicholls, T. A. Cram, and A. B. Saunders, 2006: A vortical hot tower route to tropical cyclogenesis. J. Atmos. Sci., 63, 355-386.

Nguyen, L. T., and J. Molinari, 2012: Rapid intensification of a sheared, fast-moving hurricane over the Gulf Stream. Mon. Wea. Rev., 140, 3361-3378, doi:10.1175/MWR-D11-00293.1.

Nolan, D. S., Y. Moon, and D. P. Stern, 2007: Tropical cyclone intensification from asymmetric convection: energetics and efficiency. J. Atmos. Sci., 64, 3377-3405.

Ooyama, K., 1969: Numerical simulation of the life cycle of tropical cyclones. J. Atmos. Sci., 26, 3-40.

Palmen, E., 1948: On the formation and structure of tropical hurricanes. Geophysica, 3(1), 26-38.

Pendergrass, A. G., and H. E. Willoughby, 2009: Diabatically in- duced secondary flows in tropical cyclones. Part I: Quasi- steady forcing. Mon. Wea. Rev., 137, 805-821, doi:10.1175/ 2008MWR2657.1.

Reasor, P. D., M. D. Eastin, and J. F. Gamache, 2009: Rapidly Intensifying Hurricane Guillermo (1997). Part I: Low-Wavenumber Structure and Evolution, Mon. Wea. Rev., 137(2), 603-631, doi: 10.1175/2008MWR2487.1.

M. T. Montgomery, F. D. Marks, and J. F. Gamache, 2000: Low-Wavenumber Structure and Evolution of the Hurricane Inner Core Observed by Airborne DualDoppler Radar, Mon. Wea. Rev., 128(6), 1653-1680, doi: 10.1175/15200493(2000)128<1653\%3ALWSAEO>2.0.CO\%3B2.

Rodgers, E. B., S. W. Chang, and H. F. Pierce (1994), A satellite observational and numerical study of precipitation characteristics in western North Atlantic tropical cyclones, J. Appl. Meteorol., 33(2), 129-139, doi: 10.1175/15200450(1994)033<0129\%3AASOANS > 2.0.CO\%3B2.

Rodgers, E. B., and H. F. Pierce (1995), A Satellite Observational Study of Precipitation Characteristics in Western North Pacific Tropical Cyclones, J. Appl. Meteorol., 34(12), 2587-2599, doi: 10.1175/1520-0450(1995)034<2587\%3AASOSOP>2.0.CO\%3B2.

Rogers, R., S. S. Chen, J. Tenerelli, and H. E. Willoughby (2003), A Numerical Study of the Impact of Vertical Shear on the Distribution of Rainfall in Hurricane Bonnie (1998), Mon. Wea. Rev., 131(8), 1577-1599, doi: 10.1175//2546.1. 
—, S. Aberson, M. Black, P. Black, J. Cione, P. Dodge, ... and E. Uhlhorn, 2006: The intensity forecasting experiment: A NOAA multiyear field program for improving tropical cyclone intensity forecasts. Bull. Amer. Meteor. Soc., 87(11), 1523-1537

- 2010: Convective-scale structure and evolution during a high-resolution simulation of tropical cyclone rapid inten- sification. J. Atmos. Sci., 67, 44-70, doi:10.1175/2009JAS3122.1.

- P. Reasor, and S. Lorsolo, 2013: Airborne Doppler observations of the inner-core structural differences between in tensifying and steady-state tropical cyclones. Mon. Wea. Rev., 141, 2970-2991, doi:10.1175/MWR-D-12-00357.1.

Shapiro, L. J., and H. E. Willoughby, 1982: The response of balanced hurricanes to local sources of heat and momentum. J. Atmos. Sci., 39, 378-394.

Simpson, J., 1997: Mesoscale Interactions in Tropical Cyclone Genesis, (1980), 26432661.

— C. Kummerow, W.-K. Tao, and R. F. Adler 1996: On the Tropical Rainfall Measuring Mission (TRMM). Meteorology and Atmospheric Physics, 60(1-3), 19-36. doi: 10.1007/BF01029783.

Sitkowski, M., and G. M. Barnes, 2009: Low-level thermodynamic, kinematic, and reflectivity fields of Hurricane Guillermo (1997) during rapid intensification. Mon. Wea. Rev., 137, 645-663, doi:10.1175/2008MWR2531.1.

Tao, C., and H. Jiang, 2015: Distributions of Shallow to Very Deep PrecipitationConvection in Rapidly Intensifying Tropical Cyclones. J. Climate, 28, 8791-8824.

_ J Jiang, H., and Zawislak, J. (2017), The Relative Importance of Stratiform and Convective Rainfall in Rapidly Intensifying Tropical Cyclones. Mon. Wea. Rev., 145(3), 795-809, doi:10.1175/MWR-D-16-0316.1.

Tuleya, R. E., M. DeMaria, and R. J. Kuligowski, 2007: Evaluation of GFDL and Simple Statistical Model Rainfall Forecasts for U.S. Landfalling Tropical Storms. Weather and Forecasting, 22(1), 56-70, doi:10.1175/WAF972.1.

Ueno, Mitsuru, 2008: Effects of ambient vertical wind shear on the inner-core asymmetries and vertical tilt of a simulated tropical cyclone. J. Meteor. Soc. Japan, 86, $531-555$.

Vigh, J. L., and W. H. Schubert, 2009: Rapid development of the tropical cyclone warm core. J. Atmos. Sci., 66, 3335-3350, doi:10.1175/2009JAS3092.1.

Willoughby, H. E., F. D. Marks, and R. J. Feinberg, 1984: Stationary and Moving Convective Bands in Hurricanes, J. Atmos. Sci., 41(22), 3189-3211, doi: 10.1175/15200469(1984)041<3189:SAMCBI>2.0.CO\%3B2. 
Wingo, M. T., and D. J. Cecil, 2010: Effects of Vertical Wind Shear on Tropical Cyclone Precipitation. Mon. Wea. Rev., 138, 645-662.

Xu, W., H. Jiang, and X. Kang, 2014: Rainfall asymmetries of tropical cyclones prior to, during, and after making landfall in South China and Southeast United States.

Atmospheric Research 139: 18-26.

Yu, Z., Y. Wang, and H. Xu, 2015: Observed Rainfall Asymmetry in Tropical Cyclones Making Landfall over China. J. Appl. Meteor. Climatol., 54, 117-136.

Zagrodnik, J. P., and H. Jiang, 2014: Rainfall, Convection, and Latent Heating Distributions in Rapidly Intensifying Tropical Cyclones. J. Atmos. Sci., 71, 2789-2809.

Zhu, P., K. Menelaou, and Z. Zhu, 2014: Impact of subgrid-scale vertical turbulent mixing on eyewall asymmetric structures and mesovortices of hurricanes, $Q$. J. R. Meteorol. Soc., 140(679), 416-438, doi: 10.1002/qj.2147 
VITA

YONGXIAN PEI

Born, Shenyang, Liaoning, China PR

2008-2012

B.S., Lightning Science

Nanjing University of Information Science \&

Technology

Nanjing, China PR

Meritorious in Mathematical Contest in Modeling (MCM)

2012-2016

Research Assistant

Florida International University

Miami, Florida

NASA Earth and Space Science Fellowship (NESSF)

Best Ph.D. Poster in Graduate Research Symposium, School of Environment, Arts and Society, College of Arts and Sciences, Florida International University

Doctoral Candidate

Florida International University

Miami, Florida

Second Place in Robert A. Houze, Jr. Symposium Student Poster Competition, American Meteorological Society (AMS) $97^{\text {th }}$ Annual Meeting

Robert A. Houze, Jr. Symposium Student Travel Award, AMS 97 ${ }^{\text {th }}$ Annual Meeting 